\title{
EVALUATION AND MODELING OF HUMAN POSTURE AND BALANCE IN RESPONSE TO SENSORY INPUTS IN A VIRTUAL ENVIRONMENT
}

\author{
by \\ Sumandeep Virk \\ B.Sc., San Jose State University, San Jose, CA, 2003 \\ A thesis \\ presented to Ryerson University \\ in partial fulfillment for the degree of \\ Master of Applied Science \\ in the Program of \\ Electrical and Computer Engineering
}

Toronto, Ontario, Canada, 2007

(c) Sumandeep Virk, 2007 
UMI Number: EC53581

\section{INFORMATION TO USERS}

The quality of this reproduction is dependent upon the quality of the copy submitted. Broken or indistinct print, colored or poor quality illustrations and photographs, print bleed-through, substandard margins, and improper alignment can adversely affect reproduction.

In the unlikely event that the author did not send a complete manuscript and there are missing pages, these will be noted. Also, if unauthorized copyright material had to be removed, a note will indicate the deletion.

\section{$\mathrm{UMI}^{\circ}$}

UMI Microform EC53581

Copyright 2009 by ProQuest LLC

All rights reserved. This microform edition is protected against unauthorized copying under Title 17, United States Code.

ProQuest LLC

789 East Eisenhower Parkway

P.O. Box 1346

Ann Arbor, MI 48106-1346 


\section{Author's Declaration}

I hereby declare that I am the sole author of this thesis.

I authorize Ryerson University to lend this thesis to other institutions or individuals for the purpose of scholarly research.

Author's Signature:

I further authorize Ryerson University to reproduce this thesis by photocopying or by other means, in total or in part, at the request of other institutions or individuals for the purpose of scholarly research.

Author's Signature: - 


\section{Borrower's Page}

Ryerson University requires the signatures of all persons using or photocopying this thesis.

Please sign below, and give address and date.

\begin{tabular}{|c|c|c|c|}
\hline Name & Signature & Address & Date \\
\hline & & $\ldots$ & \\
\hline & & . & \\
\hline & & & \\
\hline & & & \\
\hline & & & \\
\hline & & & \\
\hline & & $\because \therefore \therefore$ & \\
\hline & & & \\
\hline & & & \\
\hline & & & \\
\hline & & & \\
\hline & & & \\
\hline & & & \\
\hline & & & \\
\hline & & & \\
\hline & & & \\
\hline
\end{tabular}




\begin{abstract}
Evaluation and Modeling of Human Posture and Balance in Response to Sensory Inputs in a Virtual Environment

(C) Sumandeep Virk, 2007

Master of Applied Science

Department of Electrical and Computer Engineering

Ryerson University
\end{abstract}

Balance control, spatial orientation and upright posture require an accurate integration of sensory inputs. In order to understand the integration in terms of motor learning and balance improvement, experiments were conducted in a virtual environment.

The objectives of the current work were to: 1) examine the postural muscle responses, head movements, and game performance in young healthy subjects when they were exposed to unpredictable situations in the virtual environment 2) examine the extent of learning transfer that occurred in the virtual environment to the real world by means of pre and post posture tests 3 ) propose and validate the theoretical model that simulates experimental results.

Results revealed that game performance increased during training in the virtual environment. The postural test results, electromyogram and head movement data have implications in using the virtual environment in balance and vestibular rehabilitation, for alleviating simulator sickness symptoms, and enhancing spatial knowledge and memory. The model simulates the experimental results very closely and sheds light on the activation of muscles under specific situations encountered in the environment. Overall, the experimental results supported our hypothesis. 


\section{Acknowledgements}

I am most indebted to my superuisor, QDr. Tristiina MFo Gonuille for her encouragement, support and guidance. Ohe provided me with her valuable feedback and advice during the MSPDS program, at every stage and step of my research and in the preparation of this thesis. OWhout her support, this would not have been possible.

I would also like to capresss my gratiude towards my defense committee for their comments, feedbach, and valuable suggestions during, the various committee meelings.

I am thankful to OBajan, QDavid and MSatja fox their equipment in the experiment.

Qppecial thanks to my friends and lab partners, Ohudeshna, Lleo, QRanjay, Otzadeh, Matija, Pina, for all the discussion sessions we had and the "chilling" times we enjoyed.

Thanks are also due to my experimental subjects, who provided me with the valuable experimentaídala.

I deeply acknouledge the support of the Eelectrical Eengineering department throughout the wholepragram.

I thank my husband, Kraijit, and my family for their love and encouragement, without which this would have been a daunting tasks co finish. 


\section{Contents}

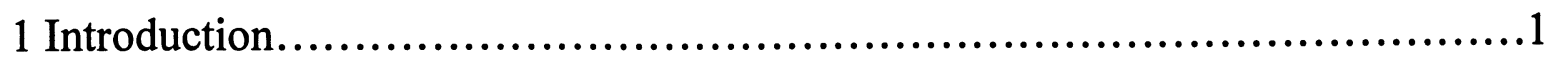

2 Background............................................................8

2.1 Postural Control and Sensory Integration ....................................................8

2.2 Virtual Reality and Vestibular and Balance Rehabilitation..............................12

2.3 EMG Signals......................................................................... 15

3 Experimental Methods..................................................16

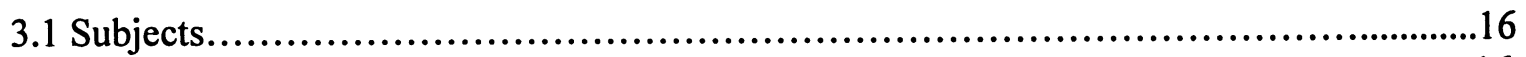

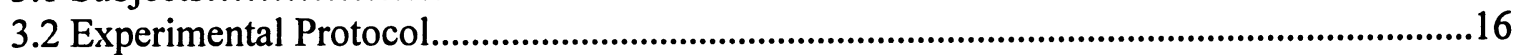

3.3 Environment...........................................................................................................18

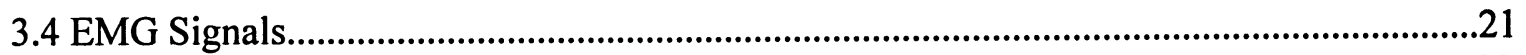

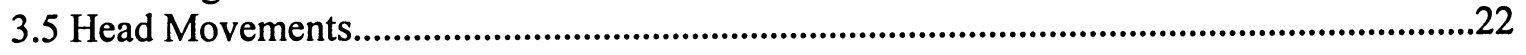

4 Results and Discussion ..............................................23

4.1 Virtual Environment Performance.............................................................................................23

4.2 Display Size and Immersiveness of VE............................................................................2

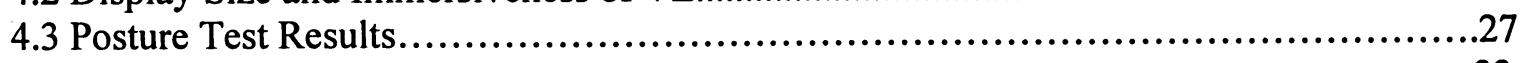

4.4 EMG Signal Analysis.................................................................................................3

4.4.1 Envelope Extraction.....................................................................................................33

4.4.2 Form Factor...................................................................................................................38

4.5 Head Movements....................................................................................................................46

5 Proposed Model Simulations...........................................49

5.1 Proposed Model.....................................................................................................................49

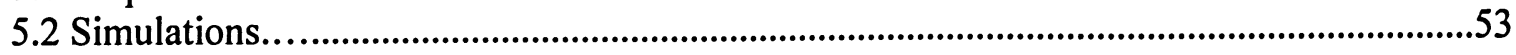

5.3 Model and Future work.......................................................................................................58

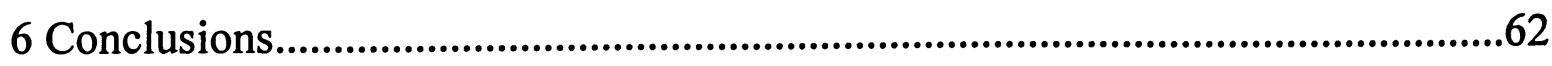




\section{Appendix A}

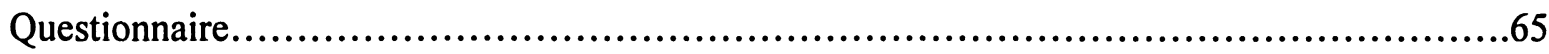

Appendix B

Simulator Sickness Questionnaire

.66

Appendix C

Consent Form.

Appendix D

Block Diagrams.

.72

Appendix E

Statistics. . .73

Appendix F

Detailed Results of EMG Analysis of Subjects. .77

Appendix G

Detailed graphs of cross-correlation of visual signal to the buffer signals. .81

Publications. .84

Bibliography .86 


\section{List of Tables}

Table 1: The Form Factor (FF) and the standard deviation (SD) in the EMG signal obtained in the pre- and post trials on a balance board for the test subjects is shown in the table

Table 2: The Form Factor (FF) and the standard deviation (SD) in the EMG signal obtained in the pre- and post trials in the tandem Romberg for the test subjects is shown in the table . .40

Table 3: Table shows the form factor in the EMG signals that show jumps in the beginning of the training (session two, week one) and at the end of the training (session eight, week three)...45

Table 4: Table shows the maximum and minimum displacement of head towards the left and right direction during the experiment in the second session . .46

Table 5: Table shows the maximum and minimum displacement of head towards the left and right direction during the experiment in the eighth session 


\section{List of Figures}

Figure 1: The various factors that help us to maintain upright stance and balance [4]...........2

Figure 2: A simplified model representing integration of various sensors, task and environment

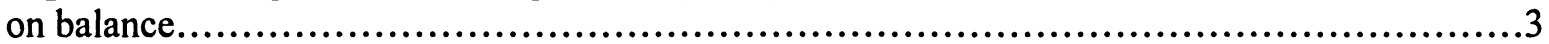

Figure 3: Models of Central Nervous System Balance control. (a) General feedback model produces motor commands $(\mathrm{u})$ that drive the body dynamics, producing motion described by the body state $x$. Sensory dynamics translate the state into sensory inputs $(y)$, feeding back to the CNS. (b) The ankle and hip muscle spindles constitute the proprioceptive sensors, semicircular canals and otoliths are vestibular sensors, and visual sensors include sensation of translation and rotation of the head. All these sensors together contribute to posture. Each of these sensors dynamically filter the state $\mathrm{x}$. (c) Direct feedback is a simple model of feedback control where sensory outputs $y$ are fed directly into a matrix of gains to produce a motor command $u$ that is essentially a weighted sum of the components of $y$. Adapted from Kuo, A.D $[34]$....

Figure 4: Graph showing the flowchart for the experiment...............................18

Figure 5: Virtual hover board environment for vestibular/balance rehabilitation. The environment encourages the user to move their head and body to navigate through the environment. The difficulty level, path and speed in the environment can be manipulated.

Figure 6: Graphical representation of the user in the virtual environment. Note that the environment does not require any markers on user.

Figure 7: The graph shows the scores of the nine users in level 1. For almost all the users the scores in the VE improved with each session. The users underwent the training in the virtual environment for three weeks, three times a week. They would always start the virtual environment sessions with level one and then move on to level two....

Figure 8: The graph shows the scores of users in level 2. For almost all the users the scores in the VE improved with each session. The users underwent the training in the virtual environment for three weeks, three times a week. They would always start the virtual environment sessions with level one and then move on to level two for the second half of the session.

Figure 9: Average performance scores fit to Equation 1 for difficulty level 1 over the 9 training sessions 
Figure 10: Average performance scores fit to Equation 2 for difficulty level 2 over the 9 training sessions. The fit from Level 1 is also plotted for comparison.......................................26

Figure 11: Balance board test results for the control subjects. The pre session refers to the first session at the beginning of the three week period, and the post session refers to the last period, at the end of three weeks. The control subjects did not undergo any training. The time was recorded to the point where a subject's balance board touched the ground or the subject reached out to grasp a surface for support. The test was discontinued after three minutes (180 seconds). Note that there is no consistent increase in the times. See above.

Figure 12: Balance board test results for the test subjects. The pre session refers to the first session, the beginning of the three week period, and the post session refers to the last period, at the end of three weeks. The test subjects did undergo the three week training. The time was recorded to the point where a subject's balance board touched the ground or the subject reached out to grasp a surface for support. The test was discontinued after three minutes (180 seconds). Note that there is a significant increase in the times. See above

Figure 13: The graph showing the Tandem Romberg test results for the control subjects. The pre session refers to the first session, the beginning of the three week period, and the post session refers to the last period, at the end of three weeks. The control subjects did not undergo any training. The time was recorded to the point where a subject lost balance or the subject reached out to grasp a surface for support. The test was discontinued after two minutes (120 seconds). Note that there is no significant increase in the times. See above. .30

Figure 14: The graph showing the Tandem Romberg test results for the test subjects. The pre session refers to the first session, the beginning of the three week period, and the post session refers to the last period, at the end of three weeks. The test subjects did undergo the three week training. The time was recorded to the point where a subject lost balance or the subject reached out to grasp a surface for support. The test was discontinued after two minutes (120 seconds). Note that there is a significant increase in the times. See above. . .30

Figure 15: The graph showing the one leg test results for the control subjects. The pre session refers to the first session, the beginning of the three week period, and the post session refers to the last period, at the end of three weeks. The control subjects did not undergo any training. The time was recorded to the point where a subject lost balance or the subject reached out to grasp a surface for support. See above.

Figure 16: The graph showing the one leg test results for the test subjects. The pre session refers to the first session, the beginning of the three week period, and the post session refers to the last period, at the end of three weeks. The test subjects did undergo the three week training. The time was recorded to the point where a subject lost balance or the subject reached out to grasp a surface for support. See above.

Figure 17: Raw signal collected from the soleus muscle. The same signal's envelope is shown in the second part. Note that it seems very difficult to obtain any useful information from the raw 
signal, whereas the envelope of the signal can more easily point to any points of interest in the signal. The data obtained is for ten seconds and is from a random stage in the experiment.......34

Figure 18: A jump recorded in the EMG for one of the subject. Note that the amplitude of the jump is relatively higher than the rest of the signal recorded in the time duration shown in this figure. .35

Figure 19: Series of Crouches. Note that the amplitude of a crouch is much smaller than the amplitude of a jump thereby implying the difference in activation of the muscle involved........36

Figure 20: In this figure five crouches are visible followed by a big jump. Note the high amplitude of the jump as compared to the crouches.............................................36

Figure 21: Figure shows EMG response to a virtual death of a user..........................37

Figure 22: The Form Factor (FF) in the EMG signal obtained in the pre- and post trials on a balance board for the test subjects is shown in the figure. Note that the FF decreases in 5 out of 8 subjects. The decrease in the FF is consistent with the findings in the literature as described in the text.

Figure 23: The Form Factor (FF) in the EMG signal obtained in the pre- and post trials in the tandem Romberg for the test subjects is shown in the figure. Note that the FF decreases for all except 2 subjects. The decrease in the FF is consistent with the findings in the literature as described in the text.

Figure 24: The figure shows the form factor calculated for eight crouches. The crouches were taken from three subjects at random times into the virtual environment. Crouch number 1 is Subject A, crouches 2 and 3 are Subject B, and crouches 4 through 8 are Subject C.

Figure 25: The figure shows the form factor calculated for six jumps. The jumps were taken from three subjects at random times into the virtual environment. Jump number 1 is Subject B, jump number 2 is subject $C$, jumps 3 and 4 are Subject $D$, and jumps 5 and 6 are Subject $A$.......43

Figure 26: The figure shows the mobility calculated for eight crouches. The crouches were taken from three subjects at random times into the virtual environment. Crouch number 1 is Subject A, crouches 2 and 3 are Subject B, and crouches 4 through 8 are Subject C.

Figure 27: The figure shows the mobility calculated for six jumps. The jumps were taken from three subjects at random times into the virtual environment. Jump number 1 is Subject $B$, jump number 2 is subject $C$, jumps 3 and 4 are Subject $D$, and jumps 5 and 6 are Subject A...........44

Figure 28: The standard deviation for both crouches and jumps for different subjects

Figure 29: A simplified postural model for the virtual environment training showing the EMG response. The input to the model is the visual signal that the user sees in the virtual environment. The database stored in memory is used to classify significant events (targets, obstacles, rings, 
launch pads etc). A desired response (jump, crouch, lateral movement etc.) is generated after cross-correlating the significant events with the visual signal. The individual gain $\mathrm{W}$, a fixed gain for every individual, scales the simulated results to match the experimental parameters.

Figure 30: Figure shows the inputs to the visual system. The sine wave represents the targets that are arranged starting from the bottom to the top and back down to the bottom. The ramp represents the take-off ramps. The series of pulses is a series of crouches to avoid obstacles. Finally the fourth event is a step input representing the launch pad ahead of the airborne rings, and immediately superimposed on it is a sine wave, which represents the approach of the first ring. It is truncated at the point that the avatar passes the ring...................................54

Figure 31: The response of the model to the input signal (as shown in Figure 30)...............56

Figure 32: The actual response of a subject as recorded in his EMG signal to the input signal (as shown in Figure 30).

Figure 33: Results of the experimental EMG and theoretical EMG (predicted by the model) superimposed on each other. The blue graph shows the EMG predicted by the model, and the red graph shows the EMG recorded during the virtual environment immersion. Note that the results obtained from both the methods are very consistent..........................................58

Figure 34: Comparator model of postural control: Sensory inputs are compared to each other for consistency, and a matrix of gains [V,C,P] is assigned accordingly. The weighted inputs are combined as $\mathrm{S}(\mathrm{t})$ which is the total sensed self-orientation $\mathrm{x}$ as a function of time $\mathrm{t}$, with noise $\eta(t)$ added. This results in a compensatory motor output through body dynamics. Feedback is provided through the senses....................................................................5

Figure D1: EMG system Block Diagram. Adapted from [69]..............................72

Figure D2: Block Diagram off Head movement system. Adapted from [70]...................72

Figure F.1: The figure shows the form factor plotted against standard deviation for jumps in the early and later trials. The crosses are jumps from earlier trial and the stars are jumps from later

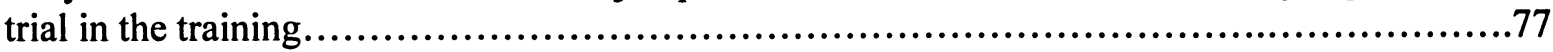

Figure F.2: The figure shows the mobility plotted against standard deviation. The crosses are jumps from earlier trial and the stars are jumps from later trial in the training.

Figure F.3: The figure shows the mobility plotted against form factor. The crosses are jumps from earlier trial and the stars are jumps from later trial in the training.

Figure F.4: The figure shows the mobility plotted against standard deviation for crouches and jumps. The diamonds are jumps and the circles are crouches. Both jumps and crouches are taken from random trials in the virtual environment.................................................79 
Figure F.5: The figure shows the form factor plotted against standard deviation for crouches and jumps. The diamonds are jumps and the circles are crouches. Both jumps and crouches are taken from random trials in the virtual

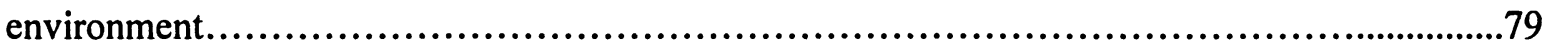

Figure F.6: The figure shows the mobility plotted against form factor for crouches and jumps. The diamonds are jumps and the circles are crouches. Both jumps and crouches are taken from

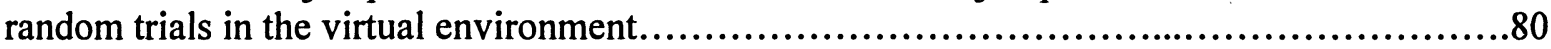

Figure G.1: Graph showing the cross correlation of visual signal with signal representing targets

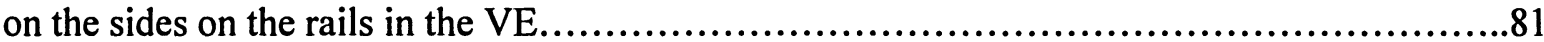

Figure G.2: Graph showing the cross correlation of visual signal with signal representing take

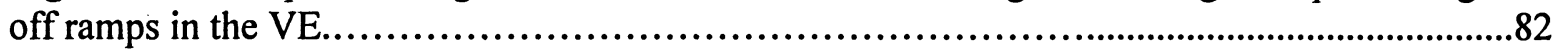

Figure G.3: Graph showing the cross correlation of visual signal with signal representing series

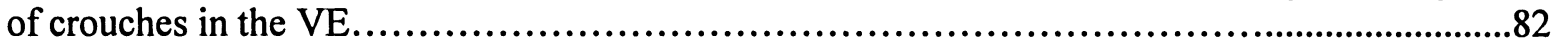

Figure G.4: Graph showing the cross correlation of visual signal with signal representing launch pad and air borne rings in VE. 


\section{List of Abbreviations}

$\begin{array}{ll}\text { ANOVA } & \text { Analysis of Variance } \\ \text { CNS } & \text { Central Nervous System } \\ \text { CMRR } & \text { Common Mode Rejection Ratio } \\ \text { EMG } & \text { Electromyogram } \\ \text { FF } & \text { Form Factor } \\ \text { M } & \text { Mobility } \\ \text { PID } & \text { Proportional Integral Derivative } \\ \text { SD } & \text { Standard Deviation } \\ \text { SNR } & \text { Signal to Noise Ratio } \\ \text { SSQ } & \text { Simulator Sickness Questionnaire } \\ \text { VE } & \text { Virtual Environment } \\ \text { VR } & \text { Virtual Reality } \\ \text { VOR } & \text { Vestibulo-ocular reflex }\end{array}$




\section{Chapter 1}

\section{Introduction}

Maintaining balance under all conditions is an absolute requirement for humans. Orientation in space and balance maintenance requires inputs from the vestibular, the visual, the proprioceptive, cutaneous and the somatosensory sensors. Each input has its own function and provides information to the CNS (Central Nervous System) based on its location and response characteristics. When in an unfamiliar environment or situation, we tend to make judgments and decisions based on past experiences which were similar to the situation at hand. As we learn and encode experiences into memory, our senses adapt. Studies show that adaptation helps in alleviating the conflicting cues and promotes re-weighting of the sensory signals. However, with certain diseases, with old age or under unexpected conditions, the balance mechanism fails to yield the desired result and the person suffers loss of balance, trips and even falls.

Balance maintenance can be quite challenging for the elderly or for people who have vestibular impairment. Falls could be catastrophic and are the leading cause of accidental death and injury in seniors. In the United States alone, falls account for 300,000 hip fractures a year with associated health care costs of $\$ 10$ Billion and can also have a significant psychosocial impact leading to social withdrawal, inactivity and immobility $[1,2]$. Falls are cited as a major reason for $40 \%$ of nursing home admissions [3]. It is important to ensure fast and accurate sensory input integration in order to keep balance responses optimal. 
The visual cues provide information about the environment, the position and motion of the head with respect to surroundings and a reference for verticality. The main role of the proprioceptive and somatosensory systems is to provide a relationship between the body segments with respect to one another (limb position) and to sense the distributed tactile input stimuli respectively. The vestibular system (located in the inner ear; we have two of them!) keeps tabs on the motion and position of the head in space. It consists of otolith organs, which detect the linear acceleration and gravity, and three semicircular canals, which detect the angular acceleration of the head. Other than these sensors, there are various elements that contribute towards our self-orientation and balance. The following Figure 1 shows these elements essential for human balance.

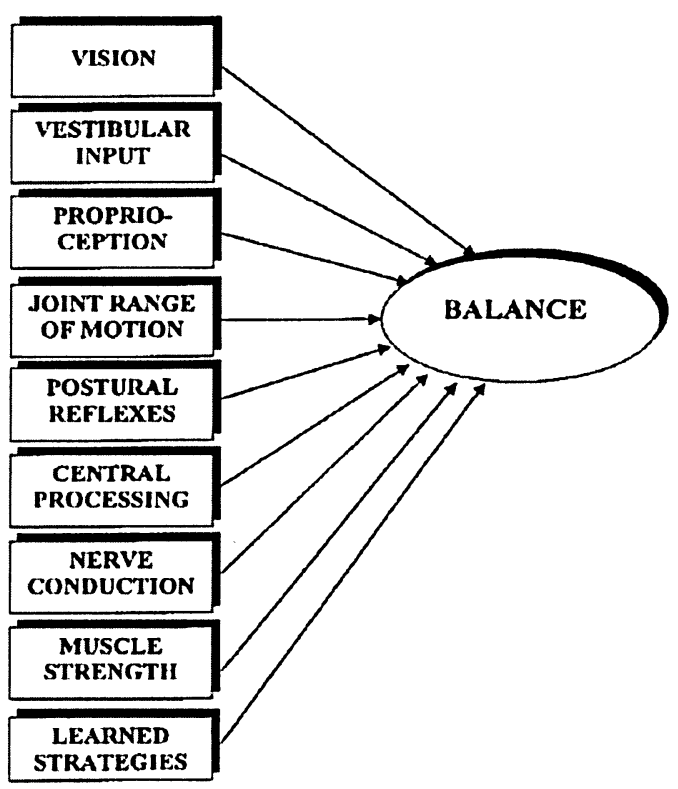

Figure 1: The various factors that help us to maintain upright stance and balance [4].

In order to understand sensory integration and balance responses, experimental data are used in theoretical models, which are then refined with further experimentation. For example to see what 
happens when the vestibular cues are absent while all other cues are intact, the responses of patients with vestibular disorders are studied. Similarly, experiments are done where somatosensory cues are disabled by asking subjects to stand on a compliant surface, such as rubber foam. To disable proprioceptive cues, the body sway referencing technique is often adopted. In this approach, the tilt angle of the support base is coupled 1:1 to body tilt angle, so that the angle of the ankle joint remains unchanged despite body sway. In these situations, it is seen that subjects rely more on visual inputs $[5,6,7,8]$. These findings clearly indicate integration (the term "integration" merely refers to summation rather than mathematical integration) and re-weighting of the inputs in the CNS. Figure 2 shows a simplified representation of the human balance control loop. For effective control of motion and balance, feedback is critical as it confirms if an intended motion is achieved or not, permits continual adjustment of output parameters and promotes stability of the control system [9].

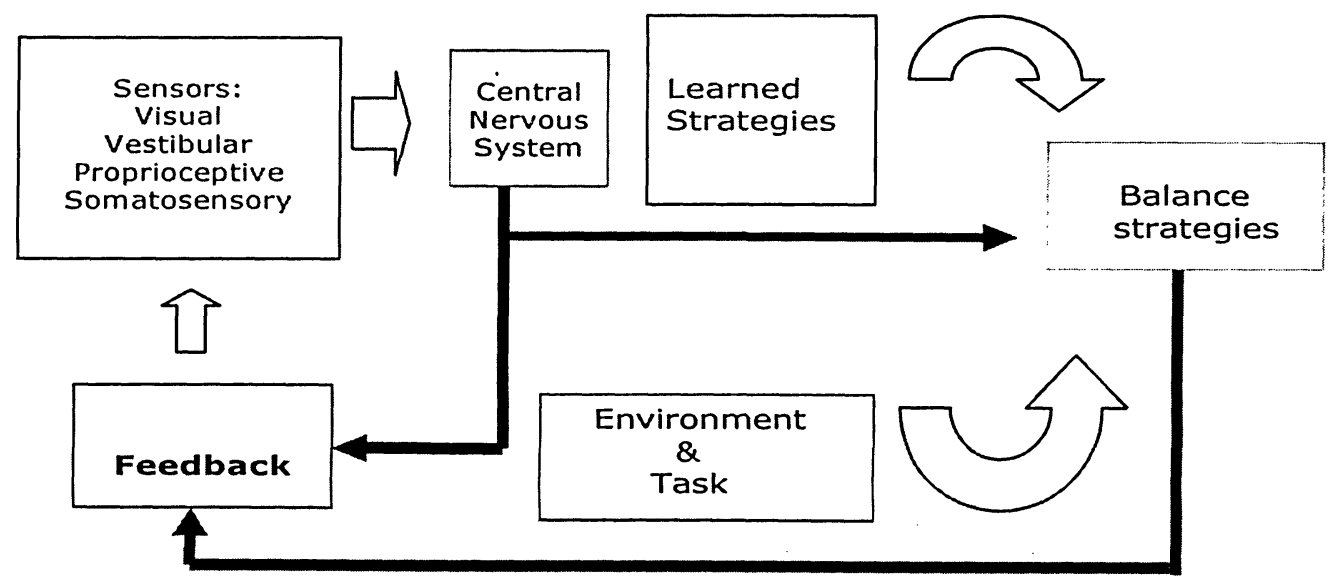

Figure 2: A simplified model representing integration of various sensors, task and environment on balance. 
Since the sensory responses deteriorate with age, studies have been done that determine the age related changes in head and eye coordination in healthy individuals. It was found that head movement gain during gaze shifts significantly increases with age [10]. As head position contributes to a stable reference for dynamic equilibrium, the significance of head movements becomes even more profound in the absence of other cues. It has been proposed that stabilizing the head in advance of body motion in the absence of visual and somatosensory cues improves the interpretation from vestibular inputs [11]. However, active head movements could also improve balance control. This could be due to an improved ability to calibrate new motion cues with respect to self-generated, known stimulus magnitudes, or it could simply be a result of improved spatial updating of the visual field. Exposure to a wide range of visual inputs and head and body movements could promote the maintenance of optimal balance responses with age [12, 13].

Apart from the elderly, people with vestibular impairment are at higher risk of falls. Approximately two-thirds of the elderly people that fall have balance problems $[14,16]$ and approximately half of these have vestibular disorders $[15,16]$. An impaired vestibular system not only poses a threat to posture, orientation and balance, but also to cognitive skills. Studies have shown that individuals with vestibular disorders exhibit a range of cognitive deficits that are not just spatial in nature, but also include non-spatial functions such as object recognition and memory [17]. Also, diversion of attention or excess load on the working memory can result in different postural strategies or even loss of balance. Quant et al. 04 showed a decrease in the magnitude of cortical responses associated with sensory processing of postural disturbances when the subject had to divert attention to a task [18]. However, previous awareness of the 
environment can reduce the stress on working memory and hence some of the responses are carried out quite automatically. Zettel et al. 05 found that gaze does not move significantly to guide stepping responses, but rather these responses are based on previously formed awareness of the subject's environment [19].

Some of the balance problems due to a deficient vestibular system could be reduced by vestibular rehabilitation exercises. The concept of re-weighting of the sensory inputs finds applications in balance rehabilitation. Interestingly, but not surprisingly, Virtual Reality (VR) is being used extensively in balance rehabilitation. The idea of using VR in rehabilitation comes from studies showing that dynamic visual inputs induce body sway in subjects. Keshner and Kenyon (2000) demonstrated in their experiments that during quiet stance, when subjects were exposed to visual rotation scenes, the upper body responded to visual-vestibular signals whereas the ankle responded to proprioceptive inputs [20,21]. Virtual reality provides an ideal environment very similar to the real world to understand the balance strategies employed by the Central Nervous System (CNS), and also helps to differentiate the behavior of various sensory inputs. It is also convenient to implement because of its low cost.

Head movements in patients with vestibular disorders were studied by Sparto et al. in a Virtual Environment. To study the biomechanical and physiological response of the inputs, postural sway was measured using force plates along with measuring head and eye movements. It was found that more weight is given to the visual inputs as compared to other inputs [22]. The immersiveness of the environment was assessed for both wide and narrow field of view displays. Rehabilitation of vestibular disorders in virtual reality provides an opportunity to combine motor 
performance and assess the effect of individual sensory inputs. The symptoms of peripheral vestibular disorders (disequilibrium and visual blurring) may be caused by abnormalities in the vestibulo-ocular reflex (VOR) gain. The VOR moves the eyes reflexively in response to vestibular inputs to maintain stable gaze. Varied visual inputs and movements of the head can play a significant role in the recovery of the VOR. Studies have shown that retinal slip (movement of the visual scene across the retina) could induce adaptation to vestibular inputs [22, 24]. Similarly, studies show the importance of head and body movements in vestibular rehabilitation and adaptation $[22,25]$. Studies by Shephard et al. indicate that the symptoms of unilateral peripheral vestibular loss were reduced through movements of the head and body in $87 \%$ of the subjects $[22,26]$. Therefore, visual motion and movements could help in vestibular rehabilitation and balance improvement. This could be easily implemented in a Virtual Environment (VE). People with vestibular disorders also complain of "visual vertigo" and "space and motion discomfort" $[22,27,28]$. In these situations the VE can provide an ideal opportunity to expose these people to different levels of visual motion and also help them to adapt to those conditions in a controlled environment. Based on the studies in balance rehabilitation, it is proposed that virtual reality can help in reducing the number of falls in the elderly or in people who are at risk of falls.

My contribution in this thesis work was to participate in the development and planning of the experiment and pilot data collection, prepare the draft ethics application, and independently conduct experiments in a virtual environment on young healthy subjects as test and control subjects. I recorded and analyzed the data, including electromyogram signals, head movements and simulator sickness (questionnaire). The performance of the subjects was evaluated for the 
potential use of that environment in vestibular rehabilitation. I developed and tested a sensory integration model, and a scaled down model to simulate the experimental results. I analyzed the head movement data, and researched and implemented different techniques for the electromyogram analysis. I prepared several manuscripts which were published, and attended several conferences including the IEEE EMBS annual conference in 2006 to present my work.

The Thesis is divided in to six chapters: Introduction, Background, Experimental Methods, Results and Discussion, Proposed Model Simulations, and Conclusions. The Background provides a preface to the subsequent chapters by: introducing postural control and orientation models, summarizing the applications of virtual reality, and reviewing the electromyogram signals. 


\section{Chapter 2}

\section{Background}

\subsection{Postural Control and Sensory Integration}

The human Central Nervous System (CNS) maintains posture and upright stance with the help of sensory inputs. As discussed, there are various sensory inputs that are responsible for our balance. How the CNS interprets the cues coming from all sensory inputs for postural stabilization has been explained with the help of various models. The exact description is not known, but attempts have been made to model the summation or integration of the various sensory inputs. The underlying principle of all these models is that the CNS dynamically allocates different gains or weights to the different inputs.

Each input has its own function and provides information to the CNS based on its location and response characteristics. However, under certain conditions like sensory impairment or with old age, the sensory systems cease to be accurate. Under these circumstances the CNS adapts itself and gives less weight to the faulty or the conflicting inputs [30] and relies more on the correct inputs. For effective balance control, it is necessary for the sensory inputs to receive feedback. For example, the visual inputs can provide feedback about the exact relative location of handrails when our balance is suddenly disturbed, or they can confirm our relative location when avoiding 
collision with an obstacle. So integration and feedback form the key elements in any model that explains the balance strategies of the CNS.

Various models have been developed with sensory weighting and feedback [6, 7, 23, 29, 33-37]. A lot of these are based on optimal estimation theory $[34,35]$ and they also address the delayed sensory information (up to $100 \mathrm{~ms}$ in the visual system) which is the time taken for sensory neurons to relay the inputs to the CNS. Many of these models see feedback as a unilateral entity, whereas others have proposed a multilateral concept of feedback. A simple example is represented in Figure 3 (adapted from Kuo (2005)). 
(a) General Feedback Control Model

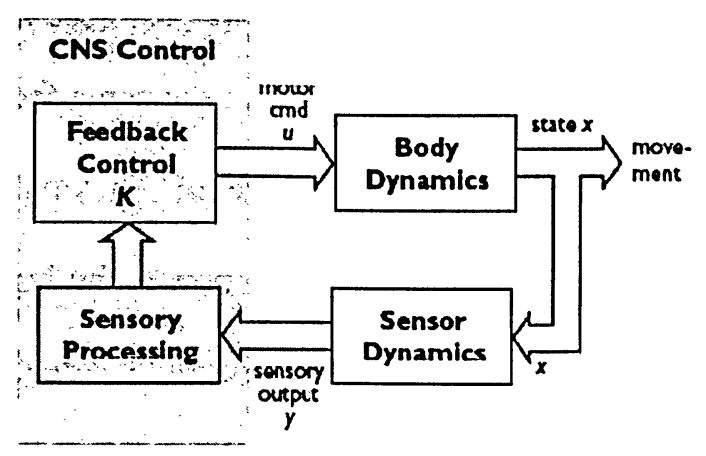

(b) Sensor Model

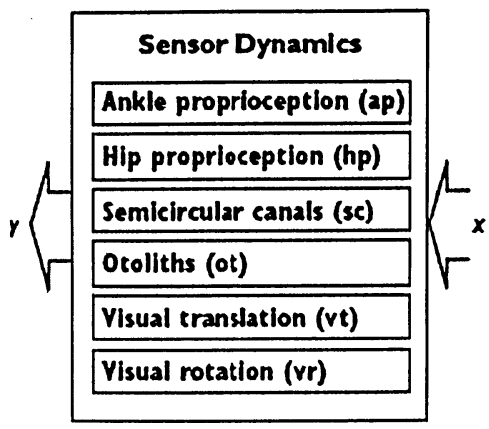

(c) Direct Feedback Model

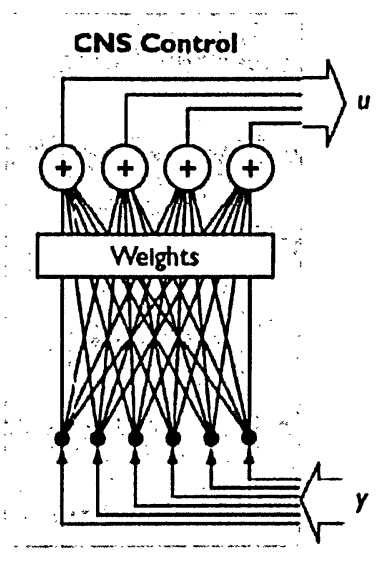

Figure 3: Models of Central Nervous System Balance control. (a) General feedback model produces motor commands $(\mathrm{u}$ ) that drive the body dynamics, producing motion described by the body state $\mathrm{x}$. Sensory dynamics translate the state into sensory inputs $(y)$, feeding back to the CNS. (b) The ankle and hip muscle spindles constitute the proprioceptive sensors, semicircular canals and otoliths are vestibular sensors, and visual sensors include sensation of translation and rotation of the head. All these sensors together contribute to posture. Each of these sensors dynamically filter the state $x$. (c) Direct feedback is a simple model of feedback control where sensory outputs $y$ are fed directly into a matrix of gains to produce a motor command $u$ that is essentially a weighted sum of the components of $y$. Adapted from Kuo, A.D [34]. 
Despite the availability and success of various models to predict postural responses to unpredictable situations and to simulate the experiments, debate still exists regarding the feedback mechanism [38]. In unilateral feedback all sensors receive the same feedback, whereas in multilateral feedback modified feedback signals are fed to different sensors. The underlying principle of multilateral feedback is very intuitive: for a correct response, the input that is faulty or not consistent with the other inputs needs the most feedback. Various researchers have also suggested a feed-forward mechanism to be an integral part of the postural control loop [39, 40]. A recent study by Musolino (2006) evaluates the effect of predictive mechanisms using cognitive tasks [38]. Many of the models utilize linear summation of the sensory inputs (i.e if one input is increased by factor of a, the output also increases by same factor), whereas others treat it as a non-linear summation (increasing one input by factor of a might lead to saturation of output).

The motion of the visual scene can induce postural reactions in humans $[21,23,29,33]$. Interesting results have been found in such studies. Studies by Mergner et al show that in normal subjects on a stationary platform, when the velocity and displacement of the visual stimulus were increased, the visual responses (visually evoked body excursion) showed saturation [21]. Similar experiments have been done with patients with vestibular impairment or by disabling proprioceptive or somatosensory cues.

The model that is proposed (see section 5.1) in this thesis was developed to simulate the experimental results. It includes the memory and spatial knowledge element to predict the EMG (electromyogram) response in the virtual environment, when the input is a moving visual scene. A more detailed discussion follows in section 5.1. 


\subsection{Virtual Reality and Vestibular and Balance Rehabilitation}

Virtual Reality (VR) is a human computer interaction environment in which the user feels immersed in the computer simulated environment and is able to conduct the tasks without fear of actually getting hurt. To the researcher, VR provides an ideal environment very similar to the real world to understand the balance strategies employed, and also helps to differentiate the role of various sensory inputs. Also in VR, the simultaneous effect of all sensory modalities and responses can be studied. There are various techniques used for the VR display; the most common are head mounted displays, wide field of view displays, and CAVEs (Computer Augmented Virtual Environments). Regardless of the virtual environment technique used, it is imperative that it gives the user some sense of presence. The sense of presence is even more important when the focus of training is on motor and cognitive abilities and skills [44]. Furthermore, the sensory inputs and behavioral responses mimic the real world experience. It is anticipated that VR will have a significant impact on balance rehabilitation in this decade [48].

As predicted, VR has been used in balance rehabilitation [20,47]. VR has been used for the assessment of vestibular influence over spatial cognitive activities such as orientation and navigation. VR also allows assessment of motor performance (body control of displacements in virtual space) [49]. Due to these features it is not surprising that VR finds many uses in balance rehabilitation and in improving balance in individuals who are at risk of falls, or have a history of falls. Some advantages of VR over other techniques are its low cost; the option to train multiple users at the same time and last but not least it makes the experience more enjoyable and reduces risk. People who might have a fear of falling or feel nervous about an activity in the real world may feel more comfortable in a virtual environment. Also, by changing the software, a person 
can be trained for any desired number of situations and skills. Depending on the performance of the user, the difficulty level can be increased or decreased. VR also helps the researcher to focus on certain aspects of the environment that are critical for training and conversely VR allows clustering (hiding or manipulating) of the prominent features of the environment. To investigate sensory integration, VR provides an ideal opportunity to examine the role of different senses on balance. It can be used to enhance or limit the role of one or more cues on balance.

Studies show that there is a high dependency on visual cues and low use of proprioceptive cues in subjects with a history of multiple falls or single falls in an occupational setting (nurses in a hospital) [45] . Also, some researchers point out that amateur soccer players rely more on visual inputs to maintain their balance as opposed to professional players. It was noted that amateur players fall more often in the field [46]. The data gathered by Vouriot et al (2004) suggests that falls victims depend on support surface and visual references, and they do not base their postural control on vestibular references. They also adopt postural control schemes that place them at increased risk of falling in specific sensory environments. Vouriot and colleagues also found that multi-fall victims demonstrated a diminished functional ability to recover balance control quickly following an unexpected external disturbance. This was hypothesized to be because of less reliance on vestibular and proprioceptive inputs [45]. In these conditions where individuals rely more on visual inputs, VR can be used to train the subjects to focus more on proprioceptive and vestibular cues, thereby decreasing the dependency on visual cues. Also, VR can be used to make the user familiar with the desired new environment, such as an occupational setting, and hence provides them with an opportunity to increase their comfort level in the new environment. 
Since balance and posture control pose major challenges to the quality of life of the elderly, various studies have been done to improve balance of the elderly using a virtual environment. Improvements on standard balance tests or centre of mass before and after the training are seen as a measure to determine the effect of training. A strength training program can be used to increase strength, muscle mass and bone density in older adults [66]. Virtual environments provide a solution for these strength training programs as the user feels more engaged in the process and treats the whole experience more or less like a "game" and hence enjoys the training. The training period typically ranges from one week to ten weeks depending on the various parameters involved in the environment and the population or on the focus of the study. Bisson et al (2006) conducted experiments on older adults in the virtual environment using a virtual environment game and found that after completing the 10 week training program, older adults improved their static and dynamic postural control, functional balance, mobility and reaction times [67]. The virtual environment they used required the subjects to lean sideways to juggle a virtual ball. The virtual environment that is used for the experiment in this thesis (see chapter 3) not only encourages the subjects to lean sideways, but also requires active movements like jumps, crouches, and movements to avoid or hit targets. The users perform multitask activities, while maintaining their postural control. The reaction time for Bisson et al (2006) showed improvements for the elderly population after four weeks of training. The findings suggest that the seniors were able to automate a postural task after the virtual training (faster reflex time). Automation and reduced reaction time are critical for seniors as reaction time is one of the most important factors to predict a fall $[63,64,65]$. 
Similar studies were also done by Bugnariu et al showing that even after one hour of virtual immersion, the CNS in older adults was able to recalibrate and adapt to changes, while improving balance capabilities [60]. The transient visual and/or surface perturbations were randomly presented to the subjects in this experiment. The results indicated that in the older adults the average number of steps taken decreased from 3 during the first ten trials to one in the last ten trials and the subjects also scored 1-2 points higher on their ability to maintain tandem stance [60].

Based on the literature review, the experiment was conducted in the virtual environment. The hypothesis was that at the end of the training: 1) the game performance of the users will increase 2) the balance test times will increase 3) the users will feel immersed in the environment.

\subsection{EMG Signals}

EMG or electromyogram is the electrical activity of firing motor units detected during muscle contraction from the surface of the skin. EMG analysis for posture and balance maintenance provides an insight into the activation and relaxation of muscles of interest, and it could also be used for the muscle conduction velocity and firing rates as well as muscle fatigue and abnormalities in muscle firing. Usually, pre -gelled electrodes (with snap conductors) are used for the human electric interface. To ensure better signal conduction and less noise, the electrodes are placed approximately one $\mathrm{cm}$ apart on the desired muscle. For postural response, generally the electrodes are placed on the soleus muscle, and/or the gastrocnemius muscle.

The EMG signals have a bandwidth of $20-500 \mathrm{~Hz}$, and amplitude of $-5 \mathrm{mV}$ to $+5 \mathrm{mV}(\mathrm{p}-\mathrm{p})$. So it is of utmost importance to amplify and filter the raw signals before analysis and processing. 


\section{Chapter 3}

\section{Experimental Methods}

\subsection{Subjects}

Seventeen subjects were recruited for the experiment: nine male, eight female; ages $22-45$ years, height $155 \mathrm{~cm}-180 \mathrm{~cm}$, weighing $50 \mathrm{~kg}-90 \mathrm{~kg}$. The subjects were randomly divided into two groups: a test group and a control group. All the subjects were right-handed (except one), had normal or corrected vision, had no medical condition affecting balance, and no history of falls. The VE training was tested in ten healthy subjects. One of the test subjects did not complete the experiment so the data are not included for that subject.

\subsection{Experimental Protocol}

The test subjects attended nine training sessions in the Virtual Environment for three weeks, whereas, control subjects did not undergo any training. On the first day a tutorial was provided as an introduction to the Virtual Reality game before the first training session was conducted. Each session was half an hour in duration. The training was given for two 15 minute blocks. After the first 15 minutes, a rest of 10 minutes was provided. The subject was then asked to navigate through the same VE (for 15 minutes), or based on the performance in the first session, the subject was moved to the next level, hence increasing the difficulty. The first training session each day was always at the lowest level of difficulty. A Simulator Sickness Questionnaire (SSQ) was completed during the break and after the training protocol (see Appendix B for SSQ). The SSQ contains 16 items on which the subjects rate their responses (ranging on a four item scale; 0 
(none), 1 (slight), 2 (moderate), 3 (severe)) [56]. The training and the testing were done with shoes off.

Standardized balance testing was done at the beginning and end of the training period. Three posture tests were measured at the beginning of the experiment and after the training period. In the first posture test--balance board test--the subject is required to maintain balance on a balance board, with feet apart, eyes open and arms on the side. The second test was the "tandem Romberg" test. In the tandem Romberg test the subject is instructed to stand heel to toe, head straight ahead, arms folded against the chest, and eyes closed. In the third posture test--one leg test--subjects were asked to stand on their right leg with eyes closed. A stopwatch was used to record the time a subject maintained the posture (to the point of losing balance or grasping a support) under the three conditions. Their EMG signals (from right leg) were recorded under the three conditions from the soleus muscle. The tandem Romberg test was discontinued after two minutes and the balance board test was discontinued after three minutes, as after these times the effect of fatigue started to show. The performance on those tests is used as a reference to see improvement in the posture control. The control subjects did not undergo any training in the VE, but the balance tests were performed on control groups in the beginning, first trial, and after three weeks.

The subjects were asked to complete a questionnaire before the beginning of the experiment, in which they reported no medical conditions affecting balance (see Appendix A). Each subject provided informed, written consent to comply with ethics approval granted by the institutional review board. (See Appendix C for consent forms). 
The following flow chart shows the course of the experiment.

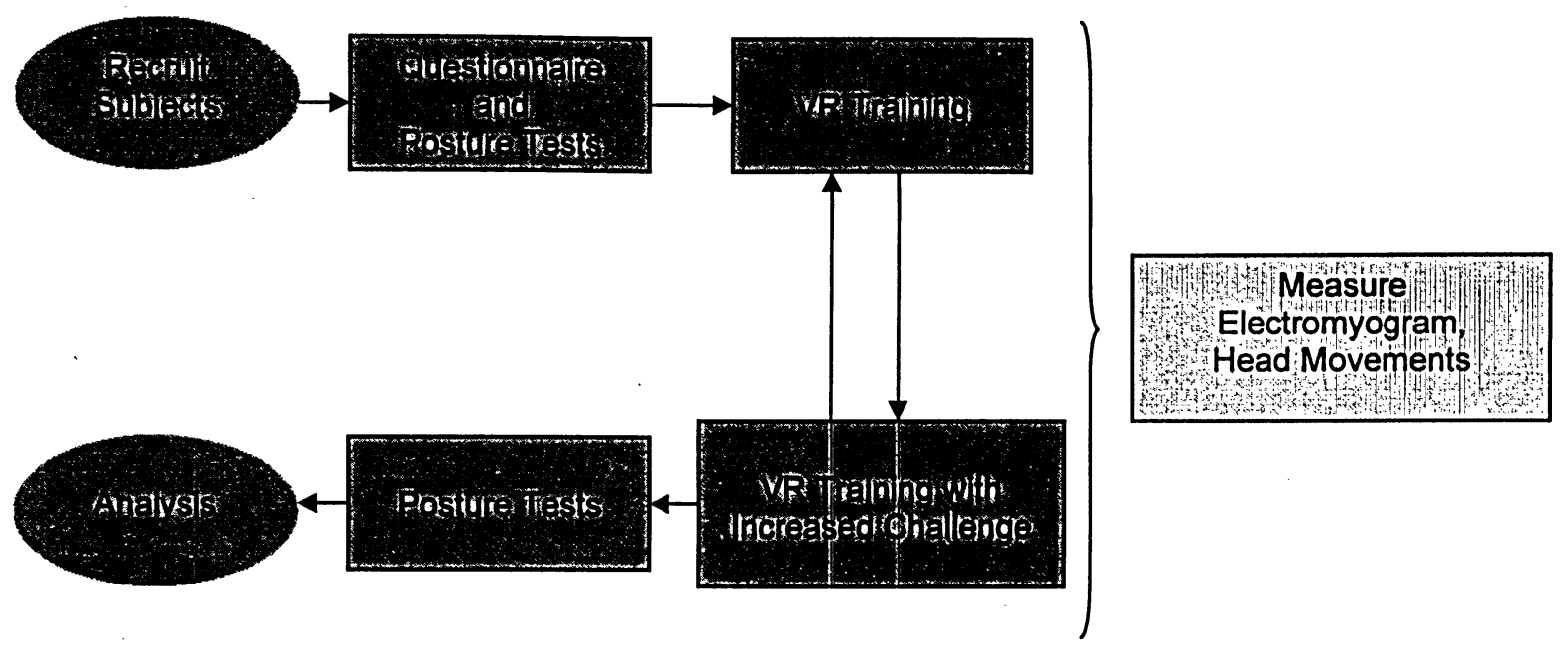

Figure 4: Graph showing the flowchart for the experiment.

\subsection{Environment}

Most of the researchers use their own Virtual Environment (VE) programs to simulate a real world environment, whereas there are many off-the-shelf programs that are available. After reviewing the types of environments used in the literature, Sony's Eye Toy on PlayStation II with the AntiGrav game was selected as a potential VE for balance training or rehabilitation. This system takes inputs from the user's head and arm movements, by imaging them with a small video camera mounted above the display, and uses software to identify and quantify head and arm movements. Each user is individually calibrated before each session, to account for height differences and range of head motion. This is a simple system operating in the lateral plane, requiring no body markers to track body position [44]. 
A regular 27" TV was used as a display. A picture of the VE is shown in Figure 5.

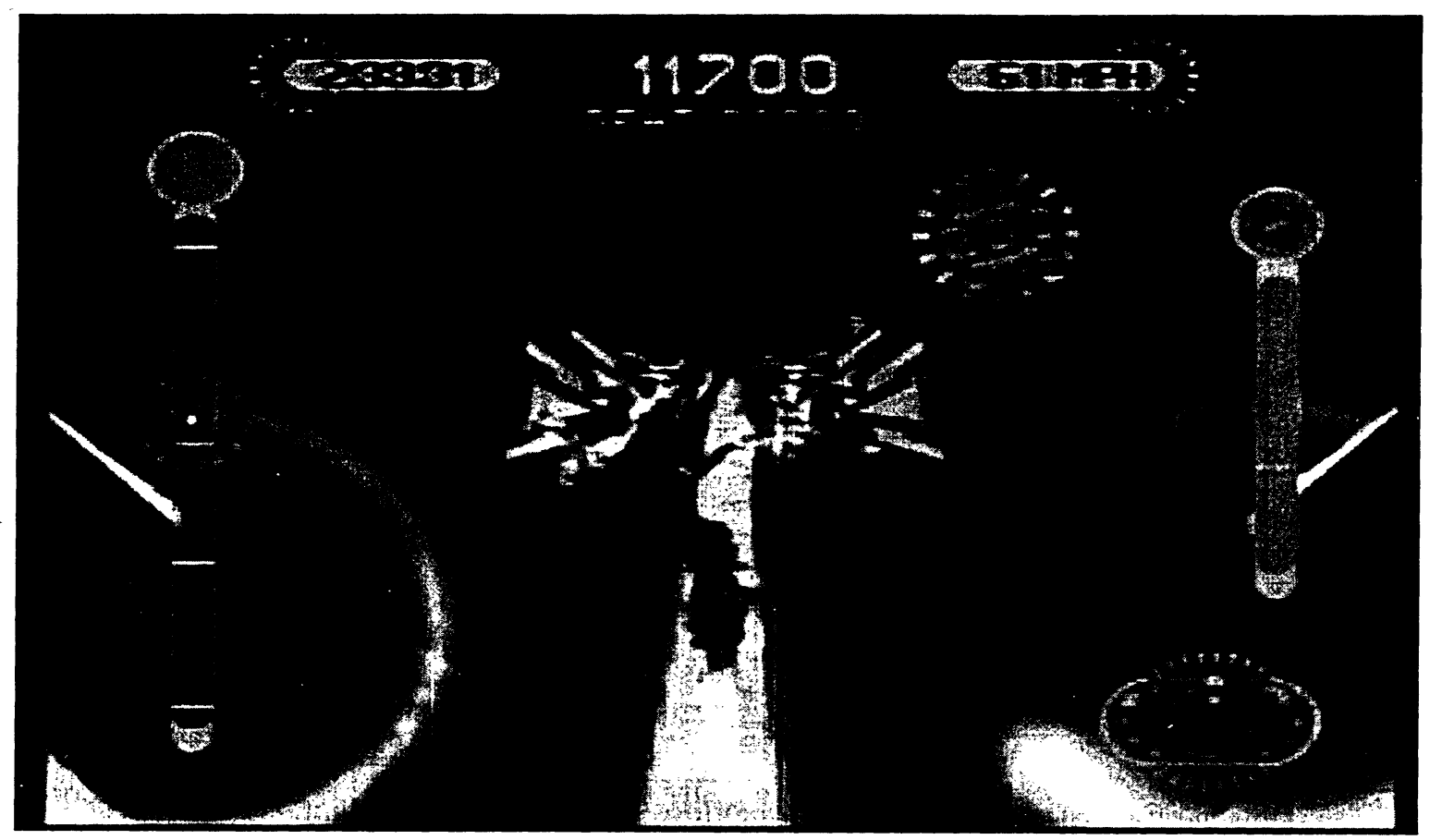

Figure 5: Virtual hover board environment for vestibular/balance rehabilitation. The environment encourages the user to move their head and body to navigate through the environment. The difficulty level, path and speed in the environment can be manipulated.

This display was validated by trying the game on two large screens and a small screen: a very large screen in a lecture hall, a portable screen with projector and a laptop screen. It was found, surprisingly, that the sense of immersion was not noticeably different, although this was not rigorously tested. Based on the similarity of the sense of immersion, the TV screen was selected, as it is the most accessible for home use. It was the intent of this study to evaluate this type of VE game for home use as a rehabilitation or training aid by the everyday user.

The user is portrayed in the game as an avatar. The user, standing in front of the display (distance based on the calibration by Eye Toy camera), must use head movements to guide the avatar on a 
hover board along roadways, over jumps, along rails and when airborne through rings, and is required to jump or duck to avoid obstacles. Game performance is measured by the number of tokens or targets that the user makes contact with by navigating the avatar through them or by contacting them with arm motions. The arm motions are directed diagonally up or down or to the side, requiring head motions to reach the targets. This requirement was hypothesized to encourage more head movements in everyday situations, such as reaching and catching as well as in balance maintenance.

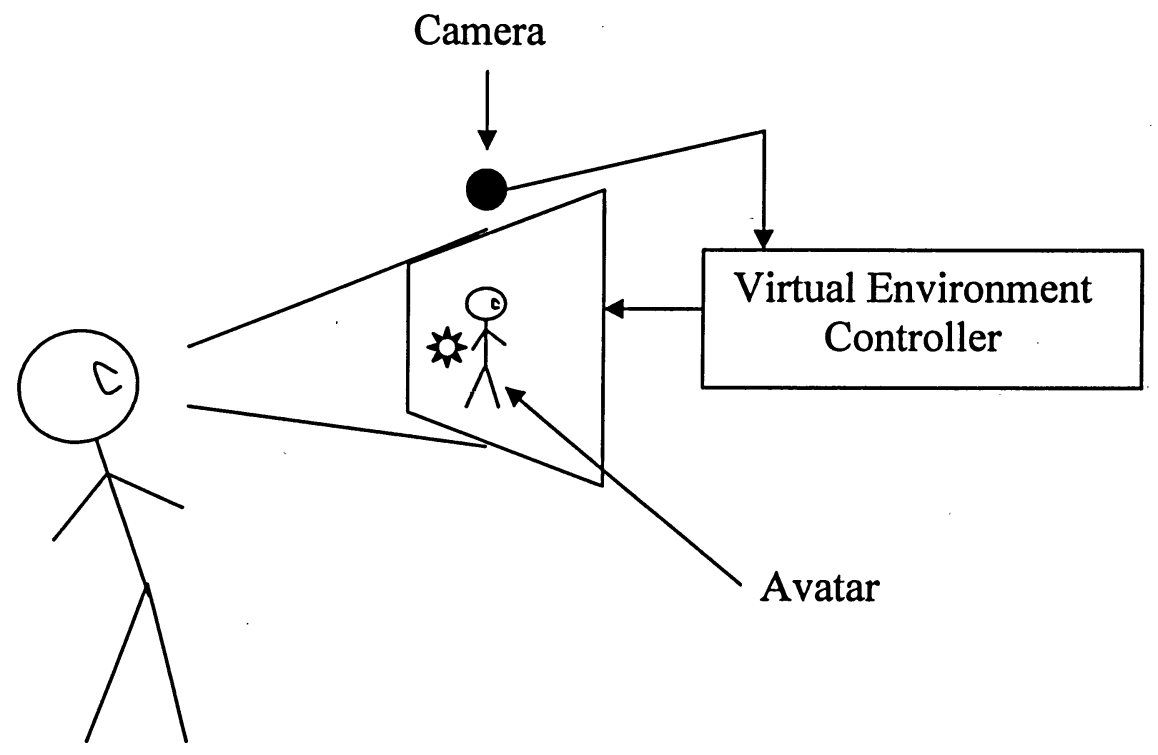

Figure 6: Graphical representation of the user in the virtual environment. Note that the environment does not require any markers on user.

Also, the game activity occurs in a very compelling immersive environment, with sound effects and "life or death" (death refers to the death of the avatar in the game) consequences in the game. It provides challenging and unpredictable situations requiring maintenance of balance and cognitive skills (such as path selection and memory) at the same time. Additional points can be 
achieved by doing "tricks": flips and rolls when airborne. The user has to visually judge the landing to stabilize the avatar before contact with the ground. There are auditory cues present in the VE, and are unidirectional.

The high-speed forward travel of the avatar creates a novel combination of visual and vestibular cues, as the vestibular inputs are limited to only those generated by the user side to side or up and down. These movements in the lateral plane have an effect on the speed and direction of forward motion, which is fed back through the user via the visual system only. While this situation does not translate directly to the real world, it decouples the learned pattern of visual-vestibular interaction, and is hypothesized to allow for re-learning or re-weighting of the combination of sensory inputs.

\subsection{EMG Signals}

EMG signals were recorded in the three posture tests and also during the virtual training sessions. The signals were amplified 1000 times and sampled at $1 \mathrm{KHz}$. To remove the artifacts from the signal, filters (a combination of band pass, low pass, derivative, comb and moving average both in hardware and software were used). To increase the CMRR (Common Mode Rejection Ratio), an instrumentation amplifier was used in the hardware design. The analysis and recording of the EMG signal was performed in the graphical interface constructed in LabView [69]. Once the signals were obtained in LabView, they were stored as text files (as matrices). (For block diagram, refer to Appendix D). 


\subsection{Head Movements}

Head movements were also recorded in each subject during the training. This gives an indication of how much the individual is moving when navigating in the virtual environment. To measure head movements, a tri-axis MEMS linear accelerometer was mounted on an adjustable headgear, which was fitted to the subject's head at the beginning of the session. The data was digitized, amplified, filtered and sampled at $1,000 \mathrm{~Hz}$ The subjects were asked to stand in a stable upright position and the head mount was then adjusted to display 0 degrees in both forward/backward and left/right directions as well to show 0 displacement in the up/down direction. After the initial calibration the forward/backward and left/right motion is captured from the initial 0 degrees to the maximum 90 degrees in any of the directions, as well as the relative offset (or vibration) from the starting position in the up/down direction. The data was displayed on a Labview interface and recorded for analysis. Three axes of head movements were recorded as text files and the data was processed and analyzed using MATLAB [69]. (For block diagram, refer to Appendix D.) 


\section{Chapter 4}

\section{Results and Discussion}

\subsection{Virtual Environment Performance}

The VE training was tested with ten subjects. One of the test subjects did not complete the experiment so the data are not included for that subject. The results of the SSQ indicate that subjects experienced slight fatigue and sweating during the trials. This was attributed to the game requiring active head and body movements to hit targets and to navigate the game, and it would be expected that the user experience some degree of fatigue. The effect of rest intervals on fatigue was not investigated, but based on initial results; a ten minute rest was felt to be appropriate between the two trials. Also, consistent with previous studies [22, 57, 58], one subject experienced slight simulator sickness symptoms (fullness sensation in the head, mild nausea and stomach awareness) after the first training session. On subsequent trials, the symptoms subsided. Two subjects felt slight headaches after training. The users felt very immersed in the environment and none of the simulator sickness symptoms were severe.

It was noticed that, with every session, the scores for the users generally increased. Figures 7 and 8 illustrate the scores in two levels of the game. Overall, there was a gradual increase in the scores over the period of the experiment. Notice that in Figure 8, which shows the advanced training level, the sessions start from session two. Subjects moved to level two after passing the 
required score (minimum of 30000) in level one. Most of the subjects were able to move to level two in second session, however, subject eight was not able to move to level 2 until session three.

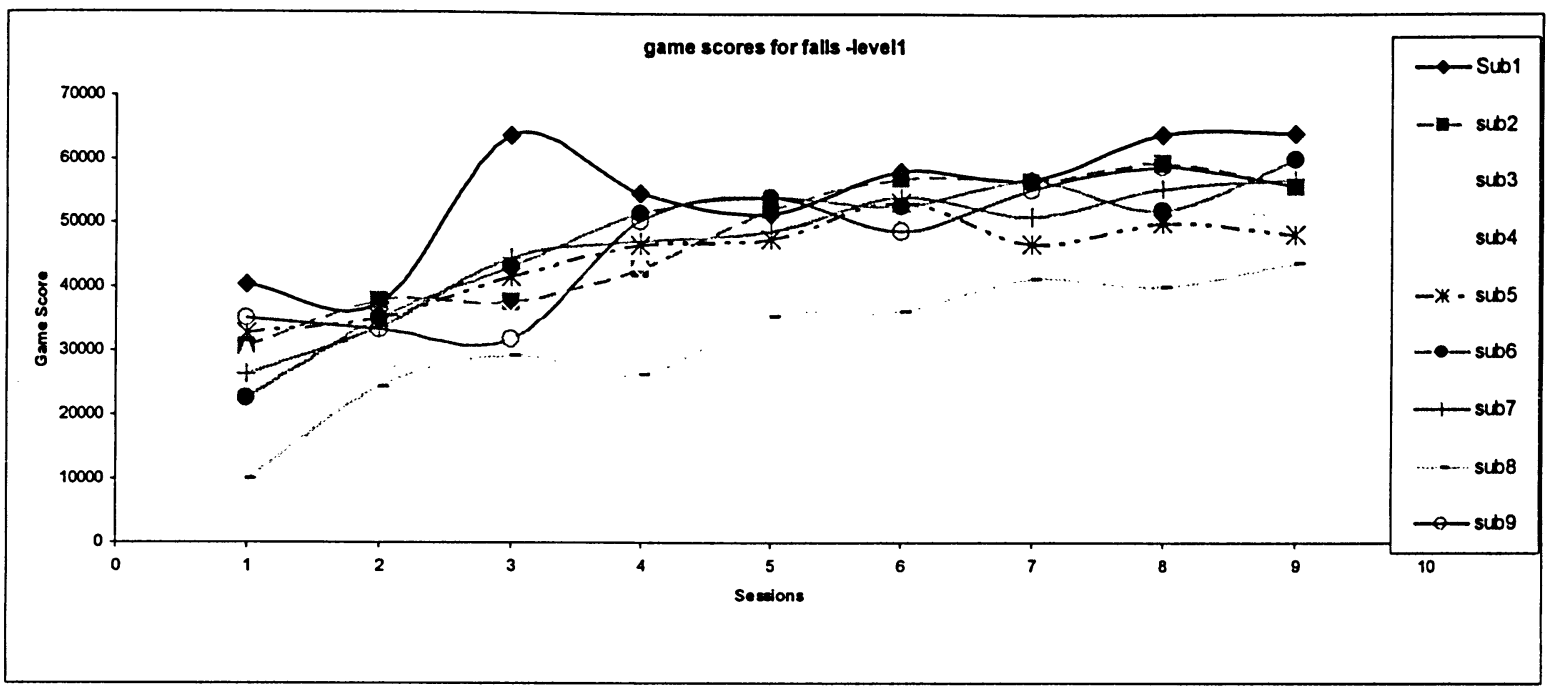

Figure 7: The graph shows the scores of the nine users in level 1. For almost all the users the scores in the VE improved with each session. The users underwent the training in the virtual environment for three weeks, three times a week. They would always start the virtual environment sessions with level one and then move on to level two.

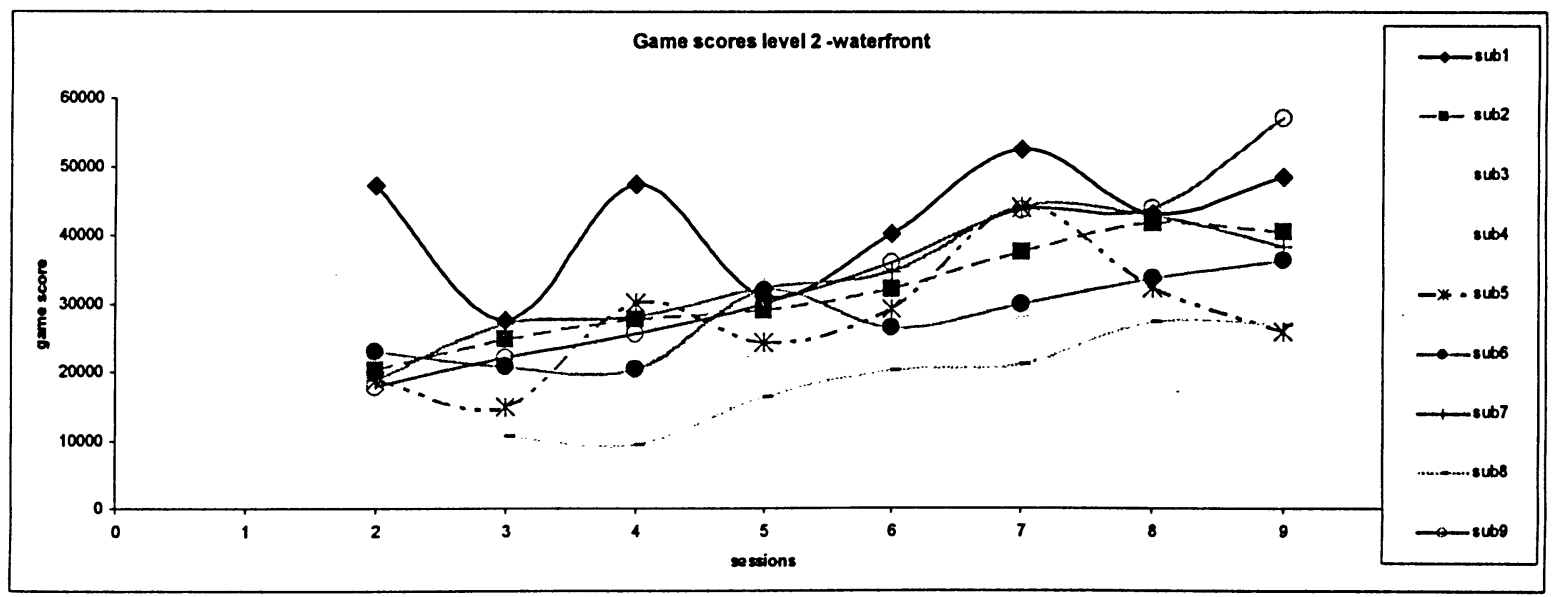

Figure 8: The graph shows the scores of users in level 2. For almost all the users the scores in the VE improved with each session. The users underwent the training in the virtual environment for three weeks, three times a week. They would always start the virtual environment sessions with level one and then move on to level two for the second half of the session. 
The Figures 7 and 8 show improvement in game scores over the nine sessions. This could be attributed to better understanding and recognition of the environment, anticipation, cognitive decisions and strategies used by the subjects. Hitting the targets require a quick and efficient response from the users, while they are speeding in the environment. This indicates an improvement in the reaction time of the users in the game. Additionally, the complexity of the environment, with several possible paths to navigate in each level, maintained the challenge throughout the training period.

The scores were averaged across all subjects for both difficulty levels and fit to an exponential [59]. The level 1 scores were fit to:

$$
y(n)=55,000-55,000 e^{-0.4 n}
$$

where $y(n)$ is the score and $n$ is the training session. The level 2 scores were fit to:

$$
y(n)=70,000-55,000 e^{-0.06 n}
$$

The average data with the fitted curves are shown in Figures 9 and 10. 


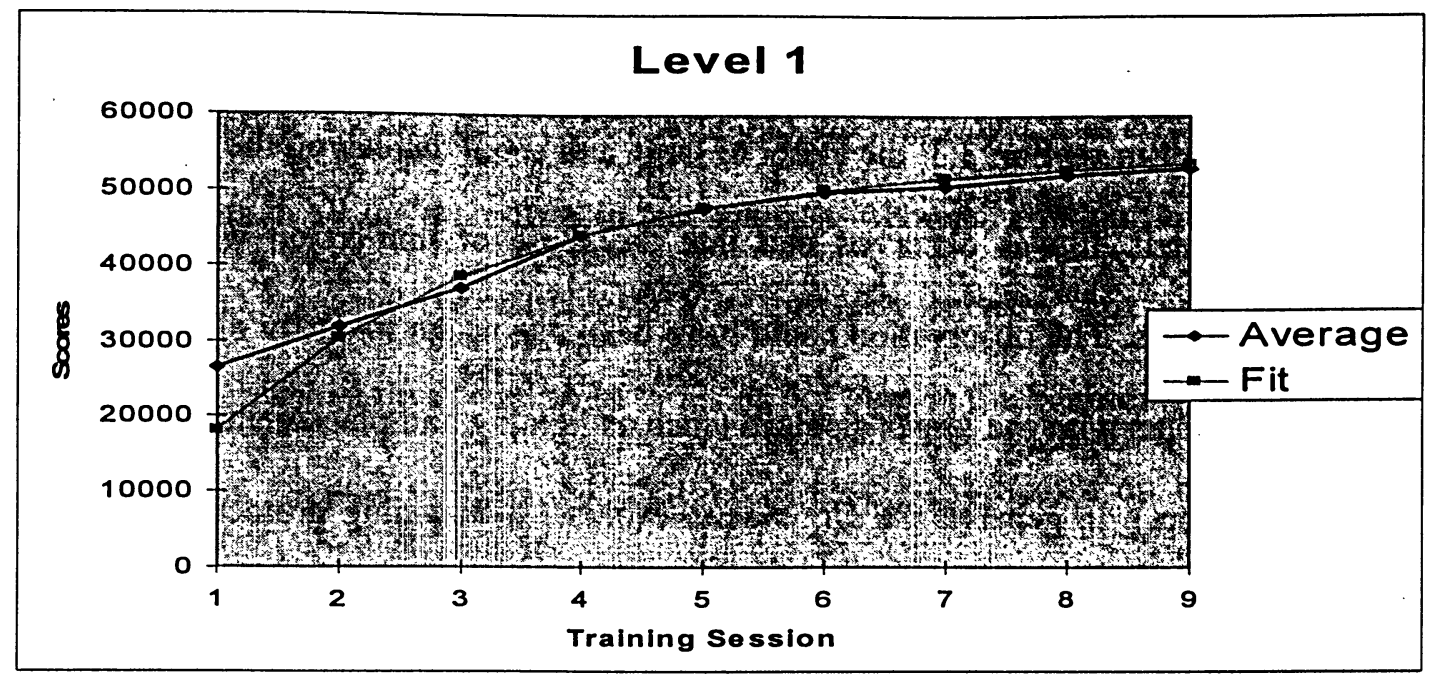

Figure 9: Average performance scores fit to Equation 1 for difficulty level 1 over the 9 training sessions. For details see equation (1).

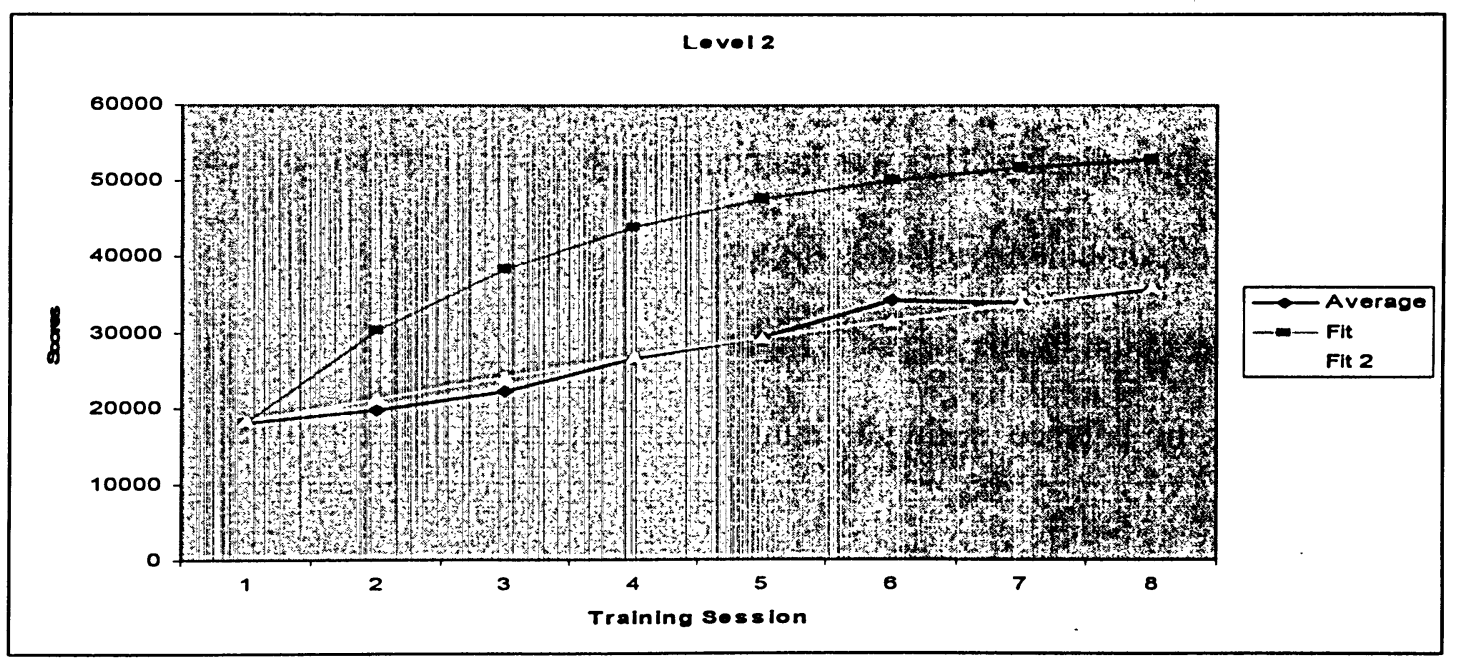

Figure 10: Average performance scores fit to Equation 2 for difficulty level 2 over the 9 training sessions. The fit from Level 1 is also plotted for comparison. For details see equations (1) and (2). 


\subsection{Display Size and Immersiveness of VE}

As discussed earlier in section 3.3, the effect of large and small display on the immersiveness of VE was investigated. It was observed that that the sense of immersion was not noticeably different in two cases. The observation could have been due to the intensity of the game as well as technical problems with the larger displays (such as lighting and placement and calibration of the Eye Toy camera). In general, the aim was to evaluate the training protocol with equipment available in a typical home environment. Large screen displays are often considered beneficial in VE training, as the peripheral cues are most conducive to the sensation of self motion. It was found that this effect is not a significant factor with this game as head movements were very similar between the large screen and the TV displays. This is due to the calibration of the Eyetoy camera being positioned at the same distance from the subject in each case. Furthermore, it is recognized that the sense of immersion may have been limited by the real world visible in the periphery around the display. This was not considered to be a detrimental factor as evidenced by the incidence of simulator sickness symptoms and the subjective reports of a significant sensation of immersion by the subjects. In fact, the peripheral cues could serve as a safety factor preventing loss of balance during training while still allowing improvement in the balance scores.

\subsection{Posture Test Results}

As pointed out in previous sections, to evaluate the effect of theVE training on a subject's postural mechanisms, three postural tests were done at the beginning and end of the three week training period. The first posture test was a balance board test, the second test was the "tandem Romberg" test, and the third posture test was one leg standing test. The tandem Romberg and one leg standing are very standard balance tests and are performed in similar studies. The 
balance board test was used in this study as it has direct similarity with the virtual environment and it was of interest to see any transfer of learning to the real world. Our hypothesis was to see some improvements in all the three tests in test subjects, whereas, no significant changes in the control population. Some of the individuals have better balance to start with, therefore, the performance of each subject was compared to his or her time in the beginning of the study. The results indicate that the test subjects did improve in their performance on balance board and tandem Romberg; however, the one leg test did not clearly show a difference. The control subjects' results did not indicate any significant improvement as expected. The following graphs illustrate these results in more detail. 


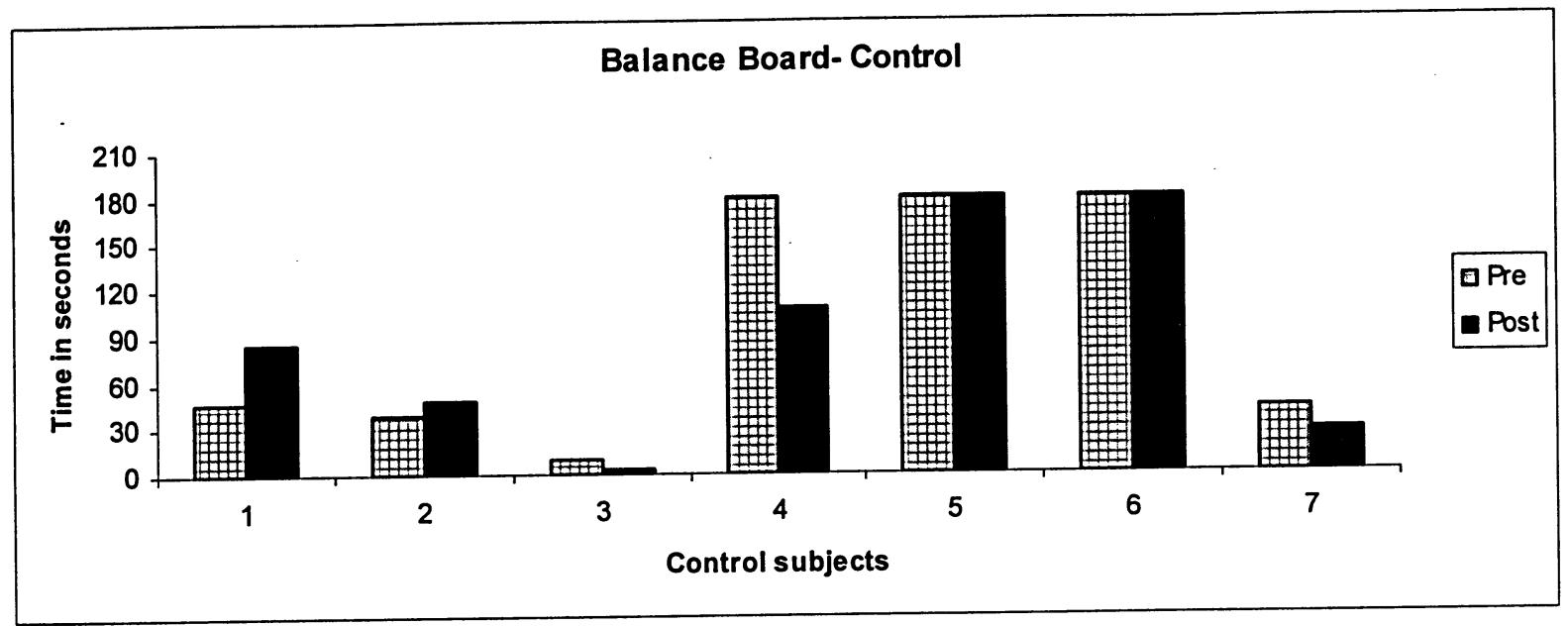

Figure 11: Balance board test results for the control subjects. The pre session refers to the first session at the beginning of the three week period, and the post session refers to the last period, at the end of three weeks. The control subjects did not undergo any training. The time was recorded to the point where a subject's balance board touched the ground or the subject reached out to grasp a surface for support. The test was discontinued after three minutes (180 seconds). Note that there is no consistent increase in the times. See above.

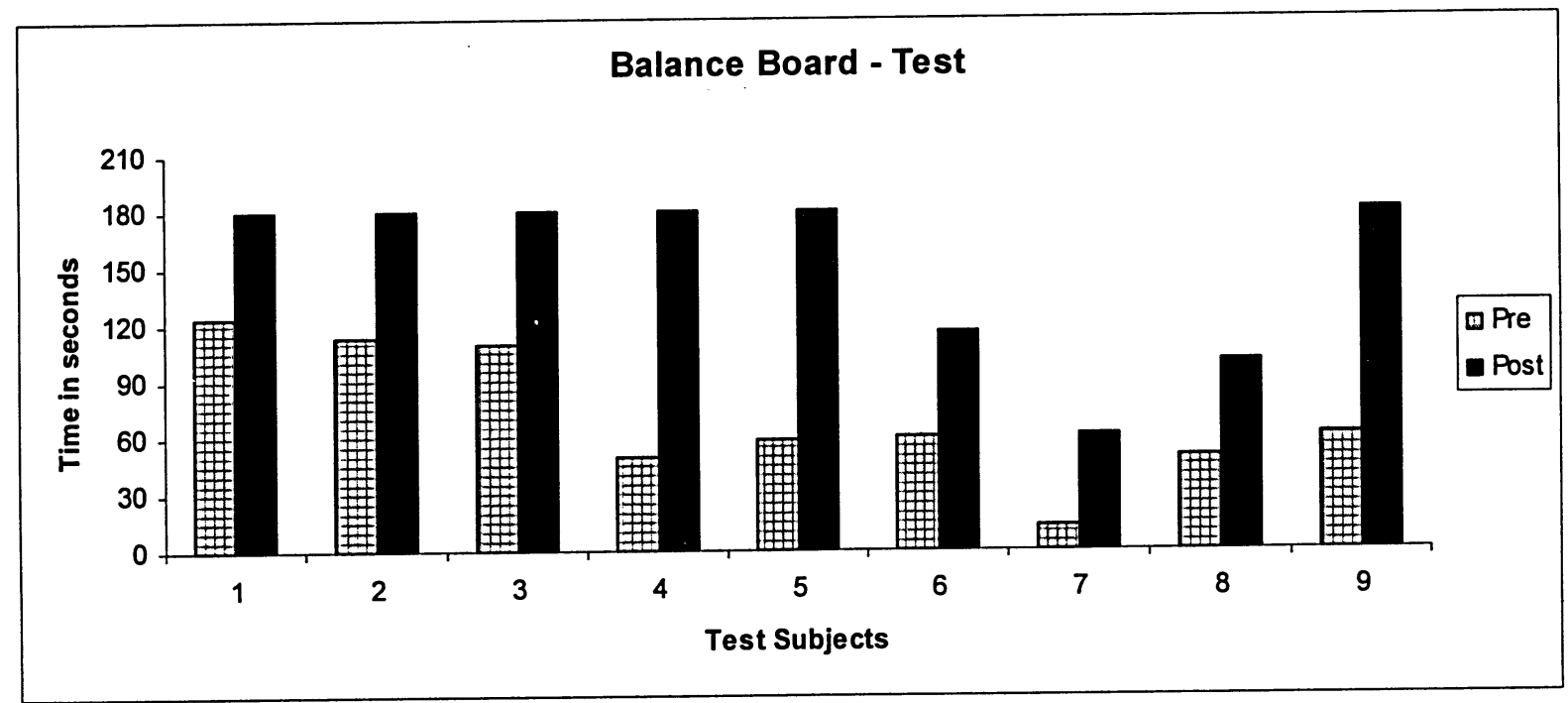

Figure 12: Balance board test results for the test subjects. The pre session refers to the first session, the beginning of the three week period, and the post session refers to the last period, at the end of three weeks. The test subjects did undergo the three week training. The time was recorded to the point where a subject's balance board touched the ground or the subject reached out to grasp a surface for support. The test was discontinued after three minutes (180 seconds). Note that there is a significant increase in the times. See above. 


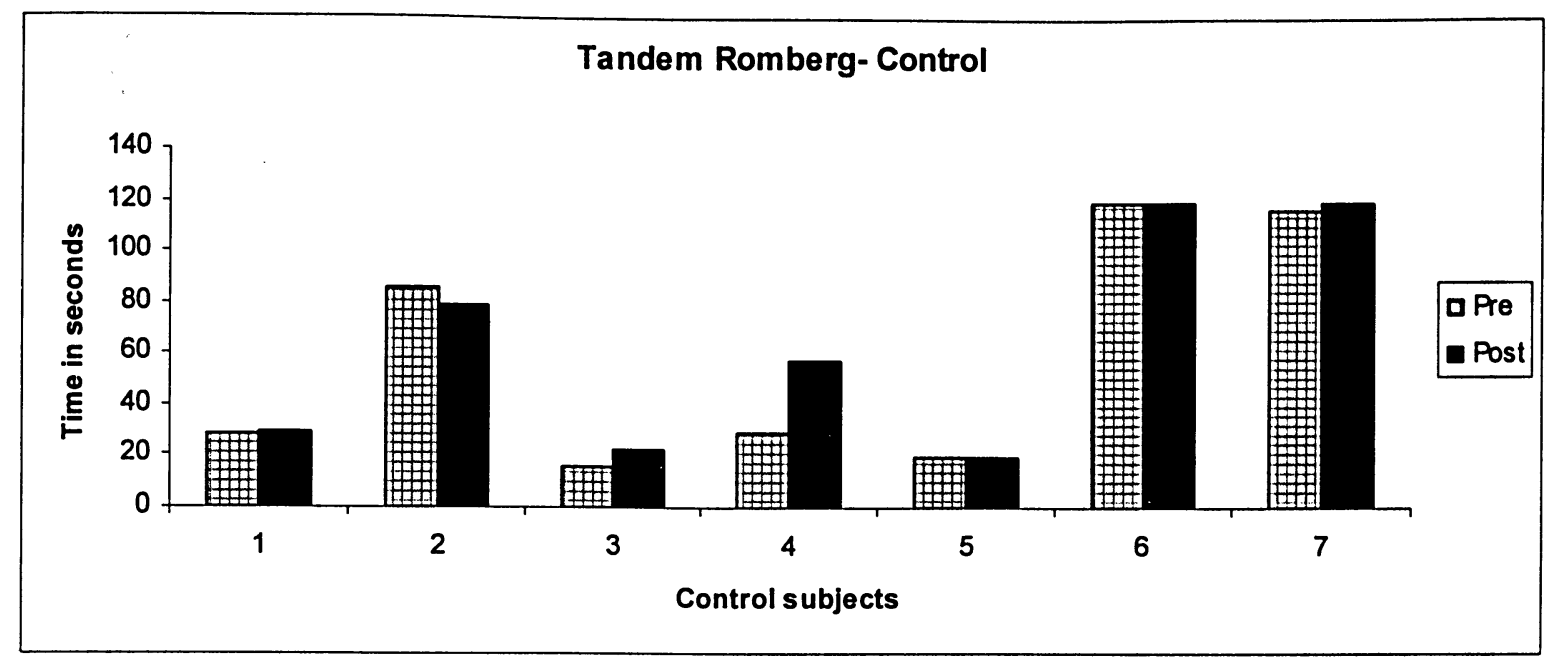

Figure 13: The graph showing the Tandem Romberg test results for the control subjects. The pre session refers to the first session, the beginning of the three week period, and the post session refers to the last period, at the end of three weeks. The control subjects did not undergo any training. The time was recorded to the point where a subject lost balance or the subject reached out to grasp a surface for support. The test was discontinued after two minutes (120 seconds). Note that there is no significant increase in the times. See above.

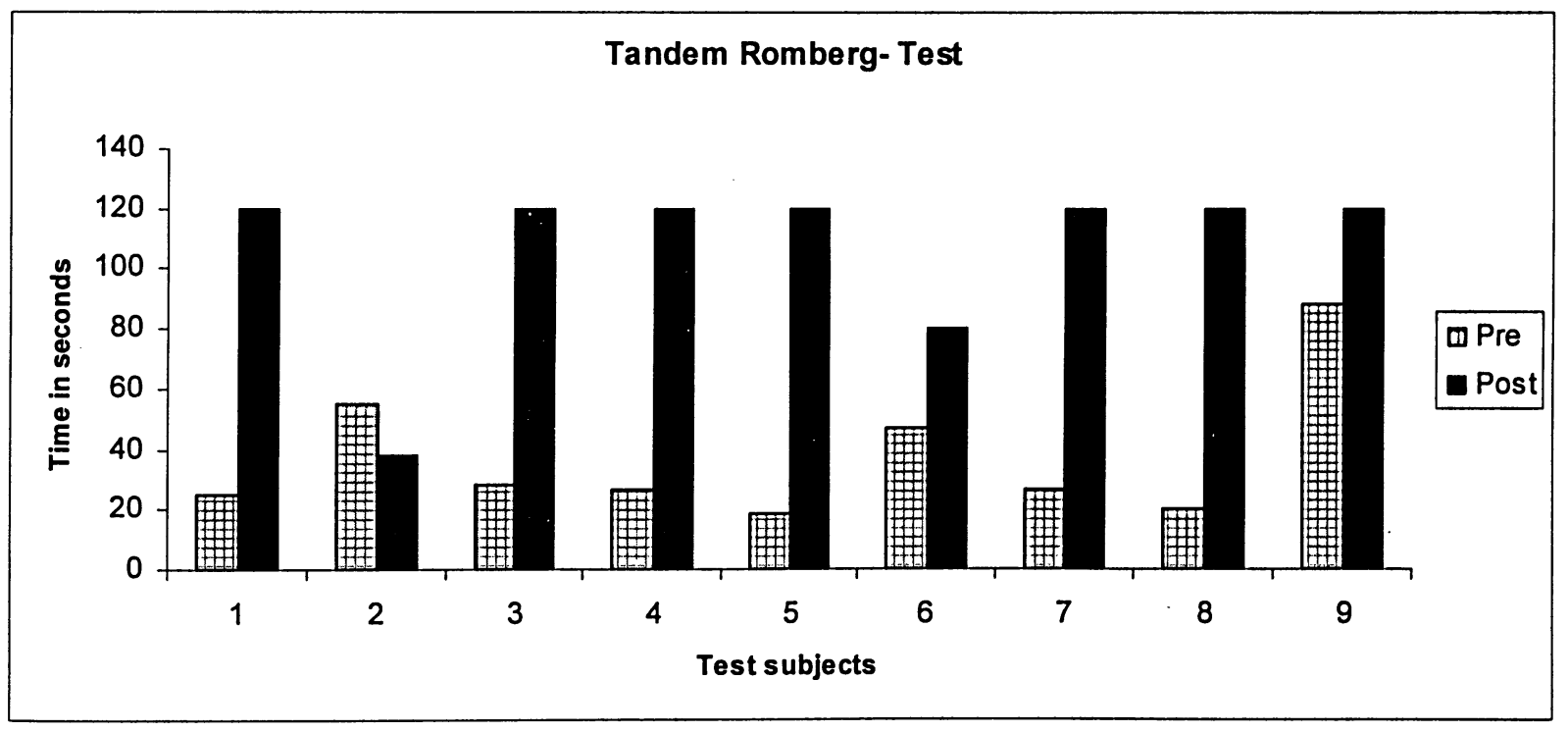

Figure 14: The graph showing the Tandem Romberg test results for the test subjects. The pre session refers to the first session, the beginning of the three week period, and the post session refers to the last period, at the end of three weeks. The test subjects did undergo the three week training. The time was recorded to the point where a subject lost balance or the subject reached out to grasp a surface for support. The test was discontinued after two minutes (120 seconds). Note that there is a significant increase in the times. See above. 


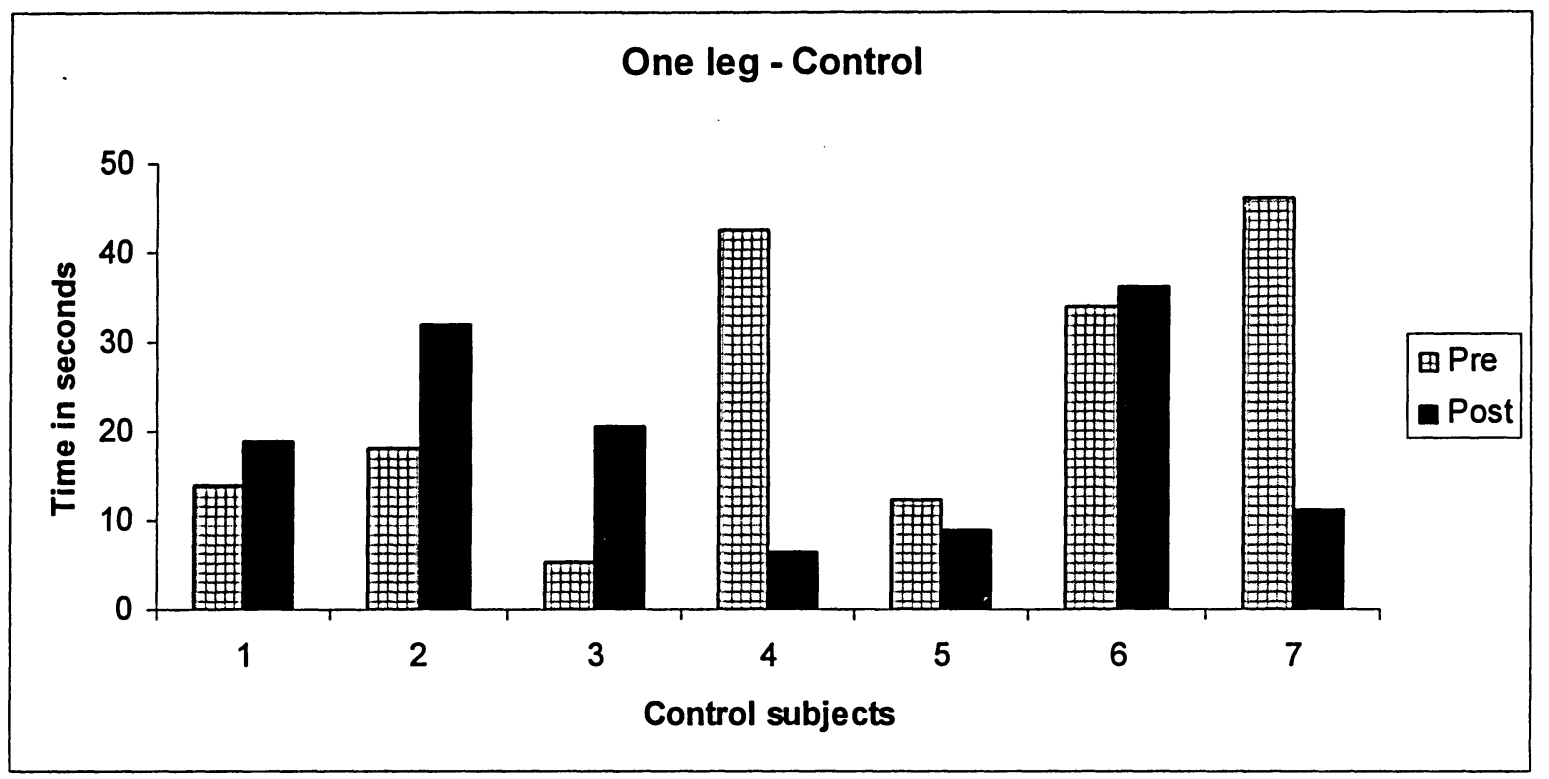

Figure 15: The graph showing the one leg test results for the control subjects. The pre session refers to the first session, the beginning of the three week period, and the post session refers to the last period, at the end of three weeks. The control subjects did not undergo any training. The time was recorded to the point where a subject lost balance or the subject reached out to grasp a surface for support. See above.

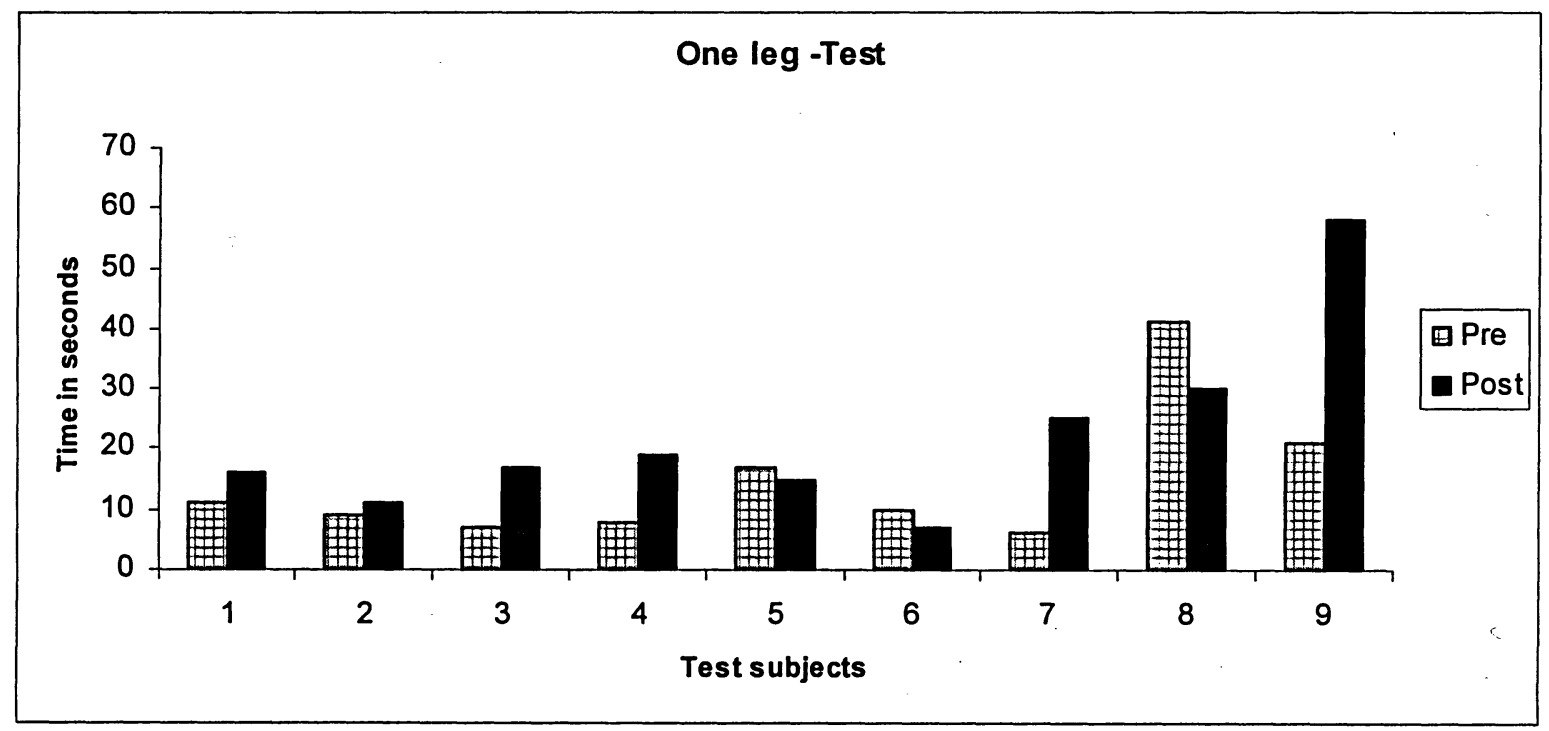

Figure 16: The graph showing the one leg test results for the test subjects. The pre session refers to the first session, the beginning of the three week period, and the post session refers to the last period, at the end of three weeks. The test subjects did undergo the three week training. The time was recorded to the point where a subject lost balance or the subject reached out to grasp a surface for support. See above. 
Performance on balance board and Tandem Romberg tests improved over the course of the training period for test subjects. Figure 11-16 shows the times that each subject was able to maintain balance. Test subjects improved on their times on the balance board and tandem Romberg. The performance on one leg test is less convincing. The subject's performance deteriorated in three of the subjects. This could be because the virtual training did not address this test as the virtual environment did not require any task on one leg.

Descriptive statistics were calculated using SPSS for appropriate demographic variables. T-test and ANOVA (analysis of variance) were done to test for significant differences in pre and post times of the postural tests. The T-test shows significance value of less than 0.05 for test subjects for all the tests, making the postural test results statistically significant. As expected, results of control population are not statistically significant (significance value greater than 0.05 ). The ANOVA analysis show the balance board and tandem Romberg times for test subjects were significantly higher $(p<.005)$, however, the one leg standing test times revealed no significant difference $(p>.005)$. For details on these tests, see Appendix E.

These preliminary data shows that this game could be used in balance improvement or balance rehabilitation. The improvement in balance board times could be attributed to the fact that the user is on the hover board in the virtual environment and the VE puts challenging demands on the lateral balance of the user with two-legged stance. Hence, the user is able to transform the VR experience in the real world in similar task conditions. The times of control subjects did not increase at all or significantly under the two tests as compared to test subjects. This suggests that the training in the VE enables the subjects to maintain their balance on a balance board and in 
tandem Romberg for longer times. The findings are consistent with study done by Bugnariu et al in which they showed that the tandem Romberg scores improved in elderly subjects even after one session of virtual reality training. The reason, they argue, is that the VE challenges subject's decision making and motor responses and hence the subjects are able to transfer their skills to the real world [60].

\subsection{EMG Signal Analysis}

After the EMG signals were obtained and recorded in LabView, they were processed in MATLAB. The signals were analyzed to extract information about the muscle's contraction and relaxation during the experiment and how these parameters changed under different conditions in the course of the experiment. The following analysis of the signals will explain in more detail how the EMG responded to movements in the virtual environment.

\subsubsection{Envelope Extraction}

The envelope of the EMG signal shows the general trends in the level of the overall activity of the muscle. It is very difficult to obtain any useful information from the raw signal, whereas the envelope of the signal can easily and readily point to any significant abnormalities or points of interest in the signal. To obtain the envelope of the signal, the positive value of the signal was taken (by squaring the signal value) at that instant, also called full wave rectification. But, this creates abrupt discontinuities at times instants when the original signal changes sign [61]. So, to remove the high frequency artifacts (created because of full wave rectification) a moving average filter was used to perform low pass filtering, leading to

$$
y(n)=1 / \mathrm{T}_{\mathrm{a}} \sum_{k=0}^{N} b_{k} x(n-k)
$$


Where $T_{a}$ is the duration of the moving average window, $x(n)$ is the full wave rectified EMG signal, and $y(n)$ is the output of the filter. The $b_{k}$ values are the filter coefficients or tap weights, $\mathrm{k}=0,1,2, \ldots \mathrm{N}$, where $\mathrm{N}$ is the order of the filter. For the analysis, a window of ten samples was used, and the tap weights therefore were unity.

To compare a raw signal with a processed signal, a raw and the processed signals are shown in Figure 17. The data obtained is for ten seconds and is from a random stage in the experiment.
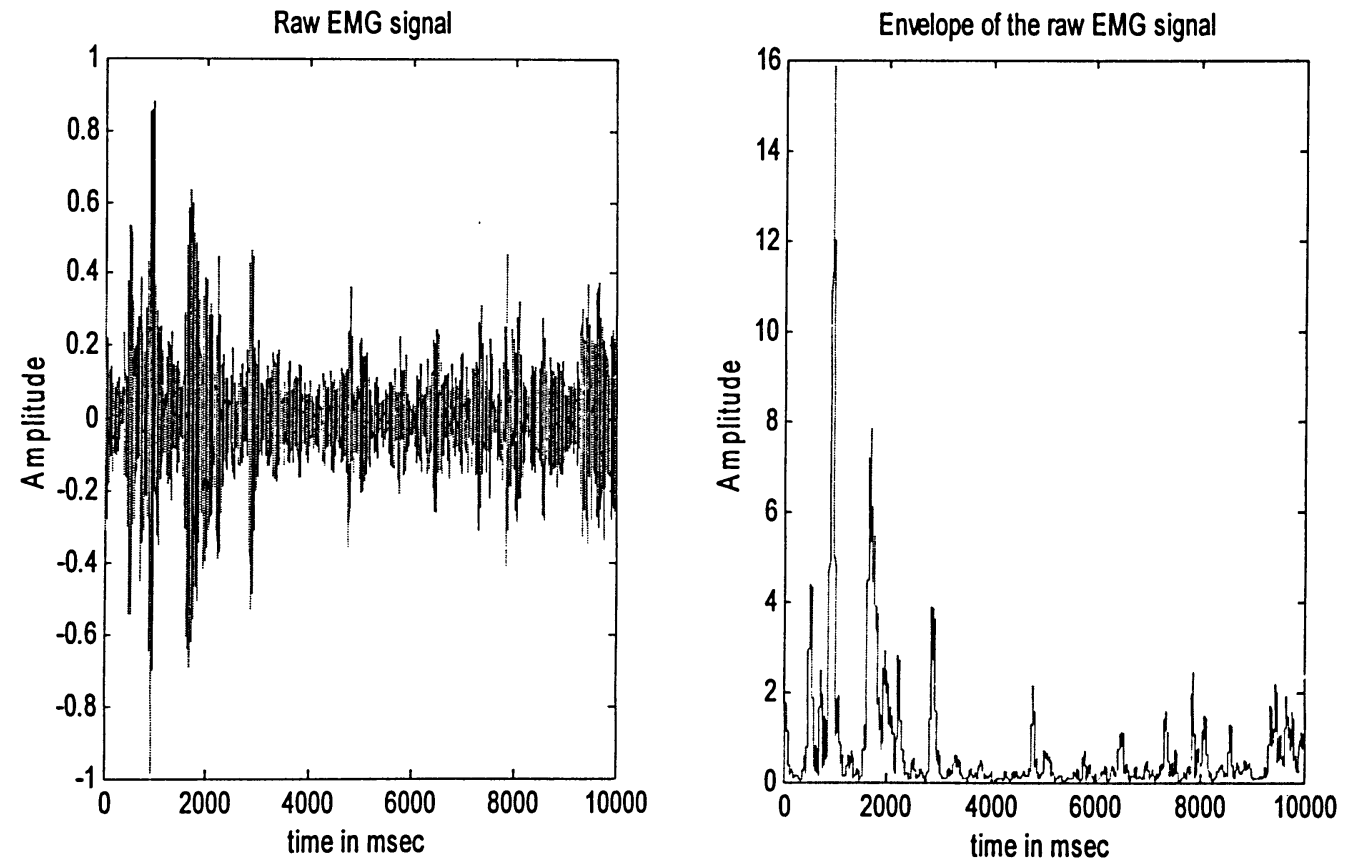

Figure 17: Raw signal collected from the soleus muscle. The same signal's envelope is shown in the second part. Note that it seems very difficult to obtain any useful information from the raw signal, whereas the envelope of the signal can more easily point to any points of interest in the signal. The data obtained is for ten seconds and is from a random stage in the experiment. 
The involvement and response of a muscle to jump and crouch is very different as seen in Figure 18 and 19. The recordings are from same subject to control frequency and amplitude variations between subjects (for details on frequency and amplitude variations between subjects refer to chapter 5). From the figures, it is evident that whereas jumps have higher magnitude, crouches have very low amplitude (in the envelope). Both put a lot of pressure on the muscle involved, however, as seen from the Figures $(18,19 \& 20)$ jumps have higher amplitude in the envelope.

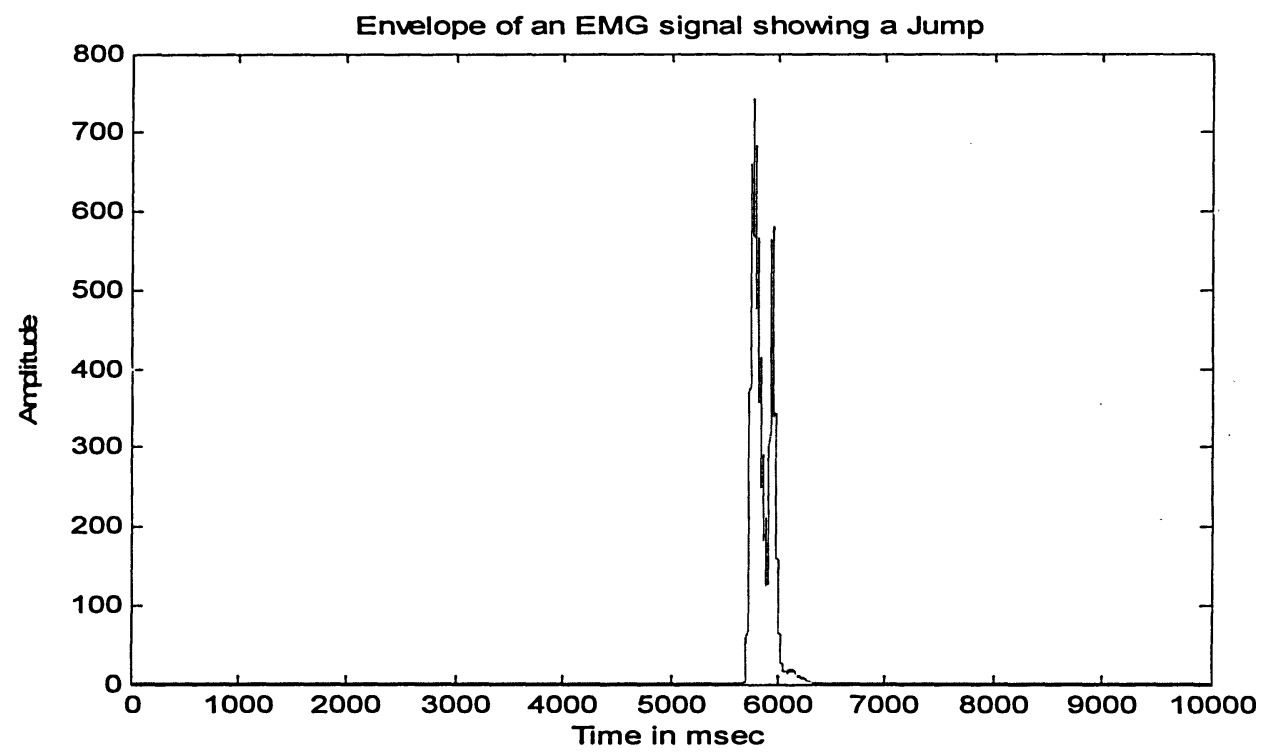

Figure 18: A jump recorded in the EMG for one of the subject. Note that the amplitude of the jump is relatively higher than the rest of the signal recorded in the time duration shown in this figure. 


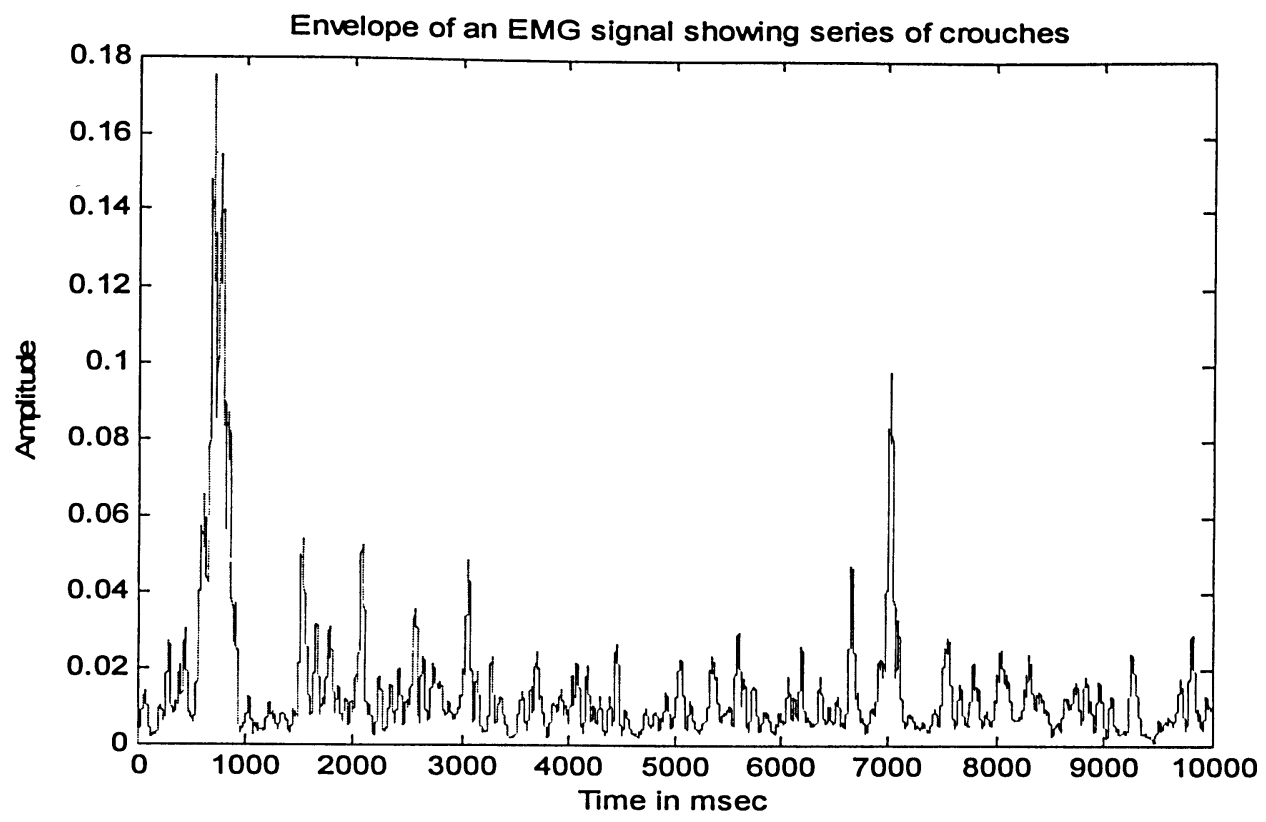

Figure 19: Series of Crouches. Note that the amplitude of a crouch is much smaller than the amplitude of a jump thereby implying the difference in activation of the muscle involved.

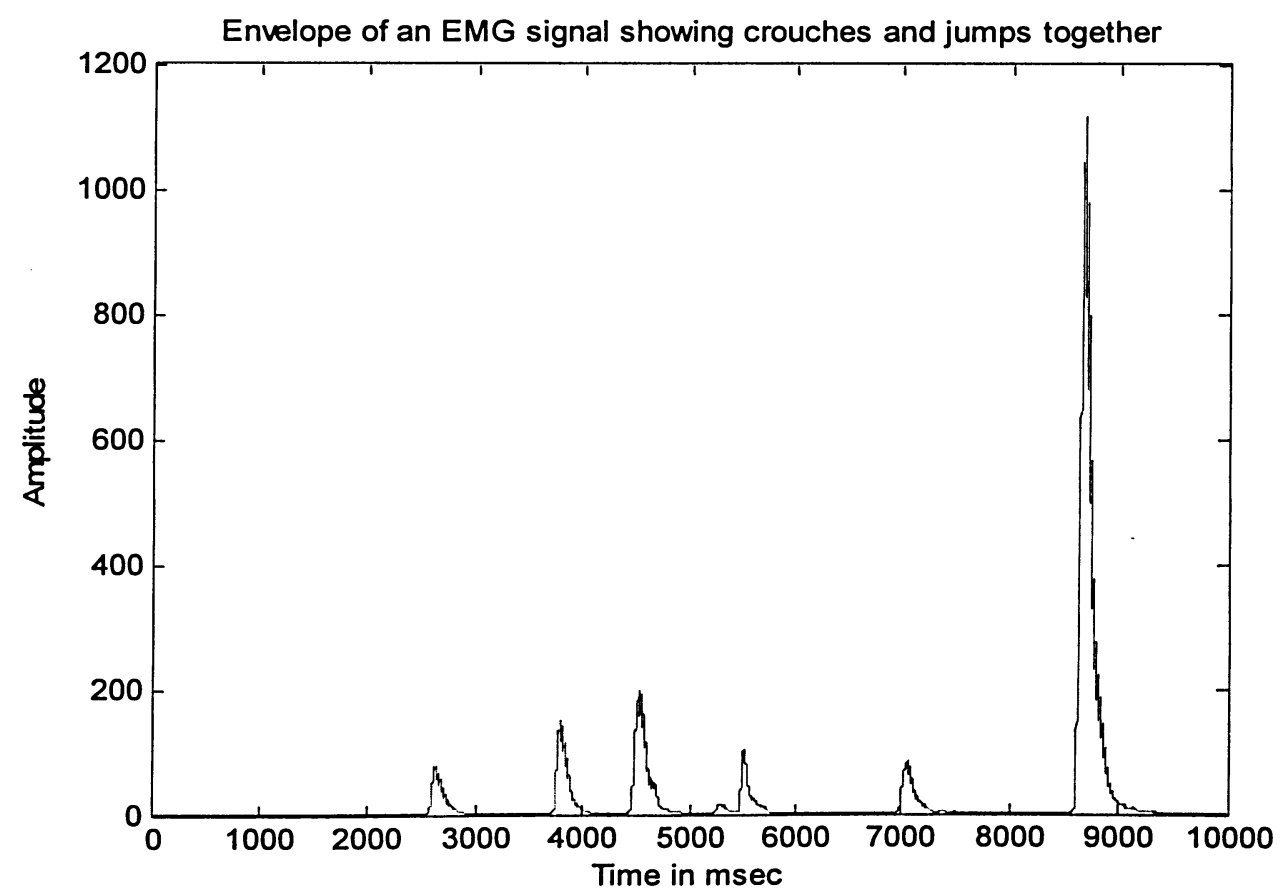

Figure 20: In this figure five crouches are visible followed by a big jump. Note the high amplitude of the jump as compared to the crouches. 
An interesting event in the experiment is the virtual death of an avatar. It is significant as it increases the anxiety and the effort of the user to save her- or himself. Figure 21 below shows EMG of one of the users in such a situation. Such a situation puts huge demand on the muscle as seen from the envelope and requires more effort for stable and effective posture in the virtual environment. This situation could be related to a real-world loss of balance.

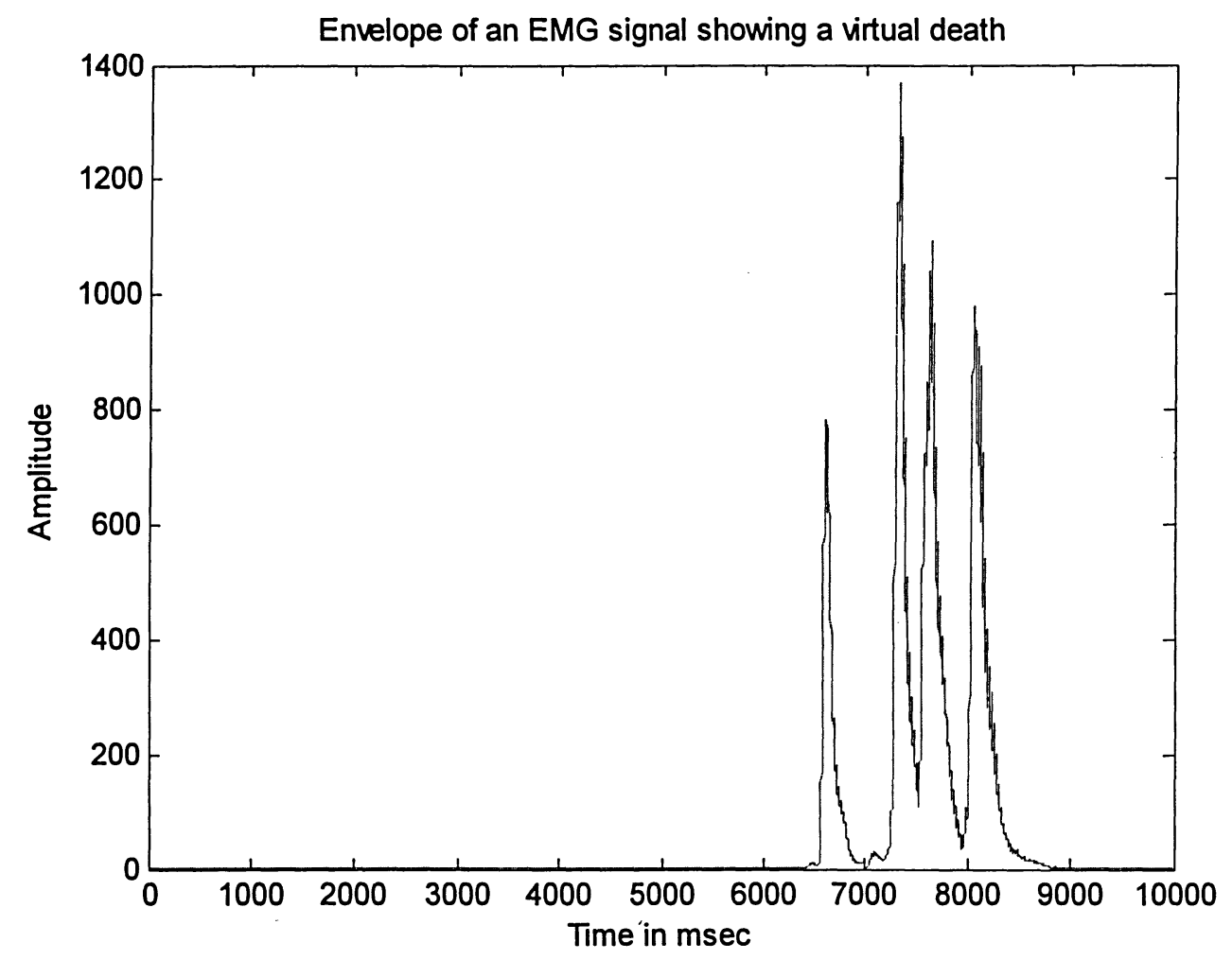

Figure 21: Figure shows EMG response to a virtual death of a user.

So, by looking at the envelope of the EMG signals (obtained during events from different subjects) it is clear that the virtual environment required different responses from the users during the course of the training. The following sections will put more light on what exactly was 
going on in terms of muscle activity and complexity during the different stages of 'action' in the experiment.

\subsubsection{Form Factor}

Another parameter that is a measure of signal activity is Form Factor and is based on the standard deviation of the signal. This method is used for segments of one second and longer and three parameters are computed [61]. The first parameter is the standard deviation [SD] (activity) of the signal. The second parameter is called mobility, $M$ (computed as the ratio of the activity of the first derivative of the signal to the activity of the original signal).

$$
M_{x}=\sigma_{x^{\prime}} / \sigma_{x}
$$

where $M_{x}$ is the mobility of the signal, $\sigma_{x^{\prime}}$ is the standard deviation of the first derivative of the signal, and $\sigma_{x}$ is the standard deviation of the signal.

The third parameter to measure activity in the muscle is called the complexity (or Form Factor, FF) and is defined as the ratio of the mobility of the first derivative of the signal to the mobility of the signal itself.

$$
\mathrm{FF}=\quad\left(\sigma_{x^{n}} / \sigma_{x^{\prime}}\right) /\left(\sigma_{x^{\prime}} / \sigma_{x}\right)
$$

where $\mathrm{FF}$ is the form factor, $\sigma_{x^{n}}$ is the standard deviation of the second derivative of the signal, $\sigma_{x^{\prime}}$ is the standard deviation of the first derivative of the signal, and $\sigma_{x}$ is the standard deviation of the signal. 
Table 1: The Form Factor (FF) and the standard deviation (SD) in the EMG signal obtained in the pre- and post trials on a balance board for the test subjects is shown in the table.

\begin{tabular}{|l|l|l|l|l|l|l|}
\hline Subject & Trial & SD & FF & Trial & SD & FF \\
\hline Sub1 & Pre & 0.0383 & 3.3766 & Post & 0.1641 & 3.405 \\
\hline Sub2 & Pre & 0.0950 & 4.2834 & Post & 0.0723 & 4.2150 \\
\hline Sub3 & Pre & 0.0831 & 4.0335 & Post & 0.0155 & 1.5463 \\
\hline Sub4 & Pre & 0.1499 & 3.9424 & Post & 0.2231 & 5.9187 \\
\hline Sub5 & Pre & 0.0236 & 4.2274 & Post & 0.1011 & 3.9268 \\
\hline Sub6 & Pre & 0.0205 & 4.6094 & Post & 0.0874 & 3.5509 \\
\hline Sub7 & Pre & 0.0253 & 5.0656 & Post & 0.0176 & 4.0802 \\
\hline Sub8 & Pre & 0.0334 & 4.2302 & Post & 0.1039 & 2.6895 \\
\hline
\end{tabular}

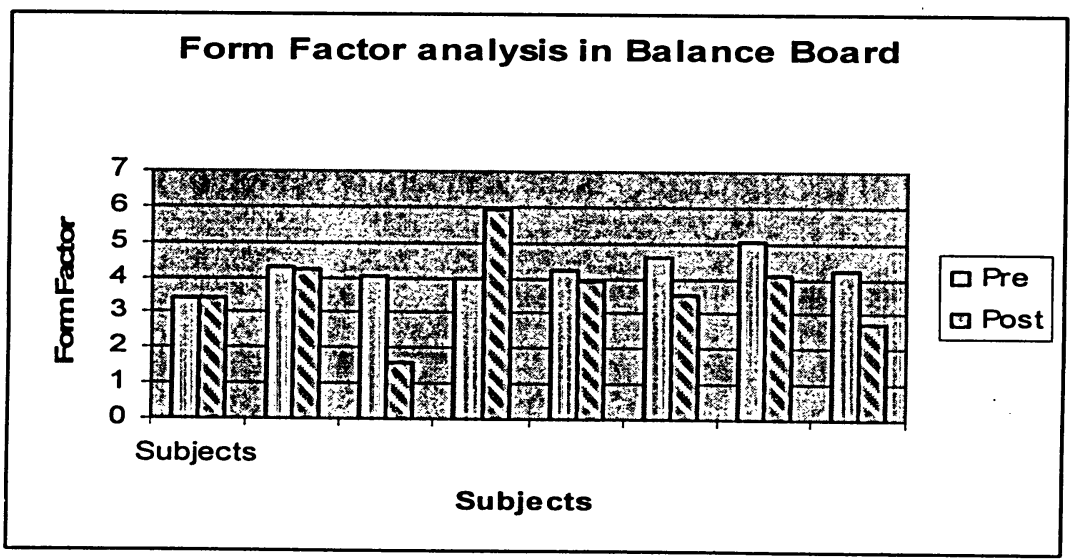

Figure 22: The Form Factor (FF) in the EMG signal obtained in the pre- and post trials on a balance board for the test subjects is shown in the figure. Note that the FF decreases in 5 out of 8 subjects. The decrease in the FF is consistent with the findings in the literature as described in the text.

Note: Data files for one of the subjects were corrupted for the post trial, so the graphs are omitted for that subject. 
Table 2: The Form Factor (FF) and the standard deviation (SD) in the EMG signal obtained in the pre- and post trials in the tandem Romberg for the test subjects is shown in the table.

\begin{tabular}{|l|l|l|l|l|l|l|}
\hline Subject & Trial & SD & FF & Trial & SD & FF \\
\hline Sub1 & Pre & 0.0078 & 1.7877 & Post & 0.0914 & 3.9879 \\
\hline Sub2 & Pre & 0.0388 & 3.8830 & Post & 0.0484 & 3.5335 \\
\hline Sub3 & Pre & 0.0503 & 3.4973 & Post & 0.0191 & 1.7402 \\
\hline Sub4 & Pre & 0.1323 & 2.7383 & Post & 0.0345 & 2.2447 \\
\hline Sub5 & Pre & 0.0393 & 3.9079 & Post & 2.4315 & 2.3875 \\
\hline Sub6 & Pre & 0.0490 & 4.9208 & Post & 0.5481 & 3.1831 \\
\hline Sub7 & Pre & 0.3082 & 1.6644 & Post & 0.0049 & 3.6760 \\
\hline Sub8 & Pre & 0.3306 & 5.1113 & Post & 0.0911 & 3.4821 \\
\hline
\end{tabular}

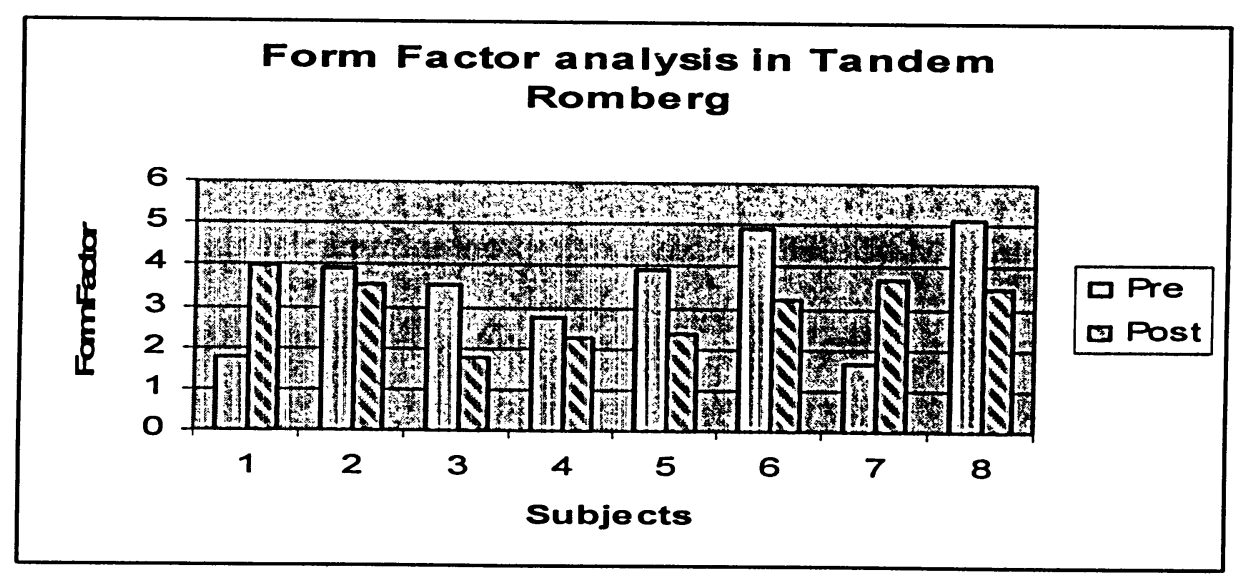

Figure 23: The Form Factor (FF) in the EMG signal obtained in the pre- and post trials in the tandem Romberg for the test subjects is shown in the figure. Note that the FF decreases for all except 2 subjects. The decrease in the FF is consistent with the findings in the literature as described in the text. 
Experiments by Osu et al. show that the there is decreased activity in the electromyogram when a subject learns the protocol in the environment [62]. The study also suggests that activity in EMG is higher in a novel environment than a normal environment. The EMG recordings in this thesis are only evaluated for the balance board and tandem Romberg tests as the one leg test did not show an improvement in times as described earlier. Refer to table 1 and 2 for the form factor calculated in the pre and post trials. For most of the subjects the form factor (activity in the muscle) decreased in balance board and tandem Romberg pre and post sessions.

It was also of interest to see any relationship between the various jumps and crouches recorded in the training. However, due to limitations of the hardware, there was a lack of samples for jumps and crouches for this set of experiment. In future, if there are enough samples of jumps and crouches for different subjects, recorded over different trials, the form factor and mobility could be used to classify and single out jumps and crouches in the EMG without actually marking them during the data collection. MATLAB or any other programming language could be used to "look" for specific responses in the EMG. This will help in achieving quantitative measures of training and ultimately relating to balance improvement in the real world.

Classification of jumps and crouches was done based on the relative amplitude of the EMG and they were extracted for different subjects in the earlier and later sessions (the envelope of the EMG was plotted and the significant events were identified by the experimenter manually; the data was classified into jumps and crouches based on the notes made when jumps and crouches were initially recorded in the experiment. This information was then used as a base to distinguish between jumps and crouches, along with the observation that the jumps had higher amplitude 
than the rest of the signal. In order to avoid any ambiguity, only those samples are discussed here in which the experimenter is certain that the significant events are jumps or crouches based on the notes). In Figure 24 and Figure 25, the crouches and jumps extracted from three subjects during random times in the training are shown and are plotted in terms of form factor. Similarly the jumps and crouches are plotted in terms of their mobility.

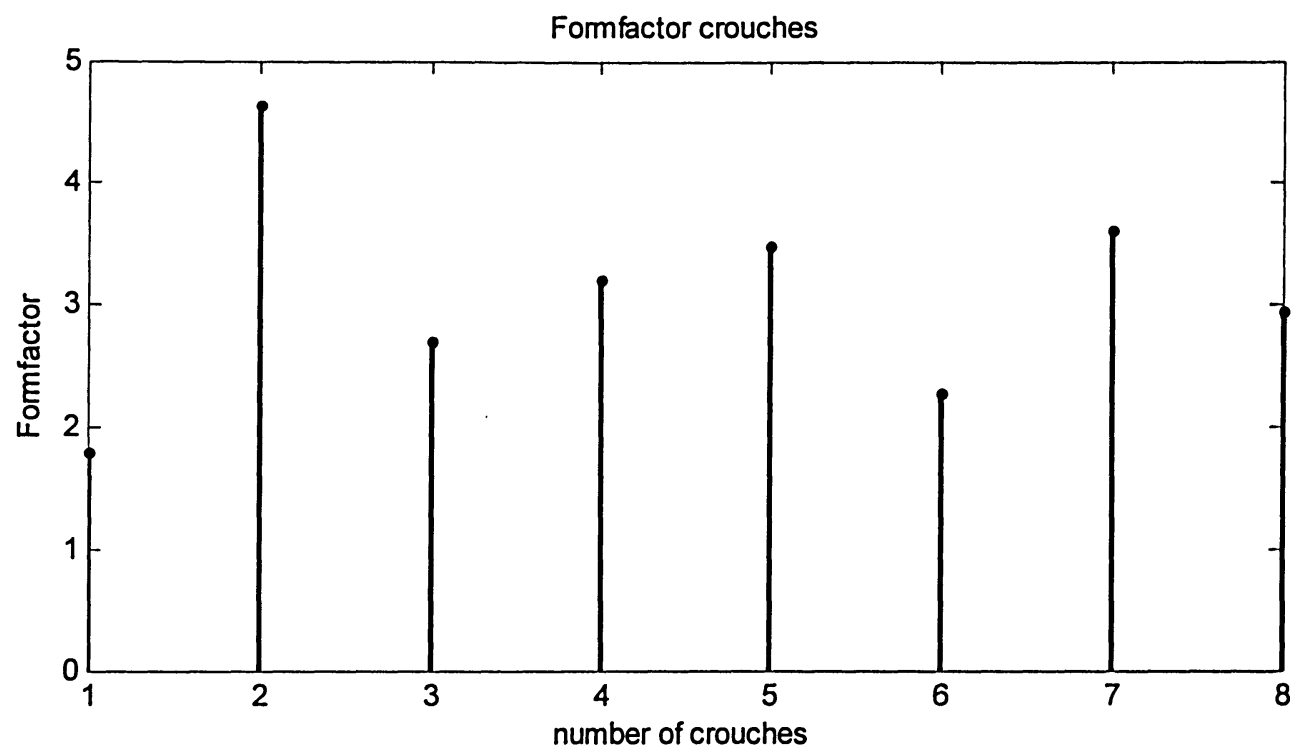

Figure 24: The figure shows the form factor calculated for eight crouches. The crouches were taken from three subjects at random times into the virtual environment. Crouch number 1 is Subject A, crouches 2 and 3 are Subject B, and crouches 4 through 8 are Subject $C$. 


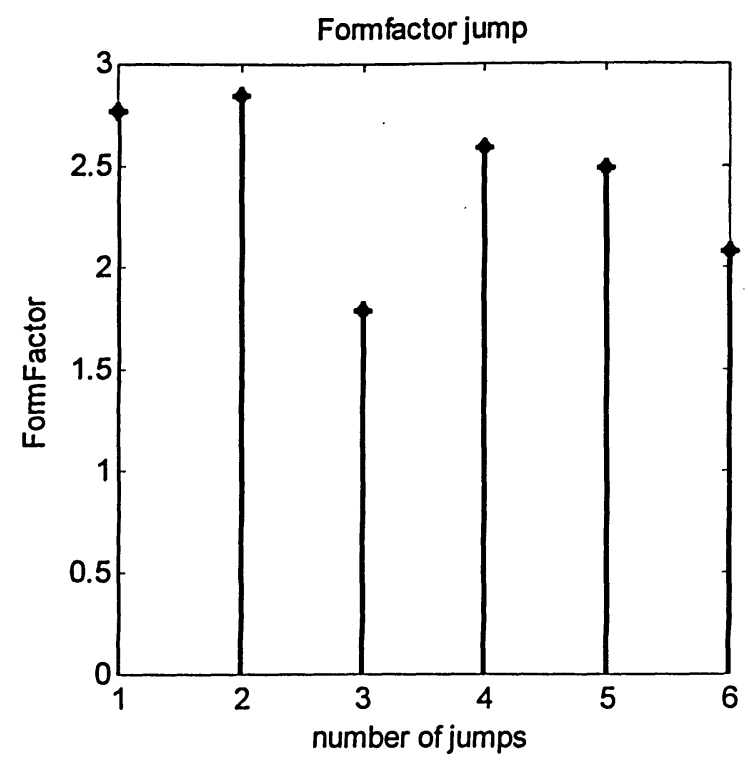

Figure 25: The figure shows the form factor calculated for six jumps. The jumps were taken from three subjects at random times into the virtual environment. Jump number 1 is Subject B, jump number 2 is subject $C$, jumps 3 and 4 are Subject D, and jumps 5 and 6 are Subject A.

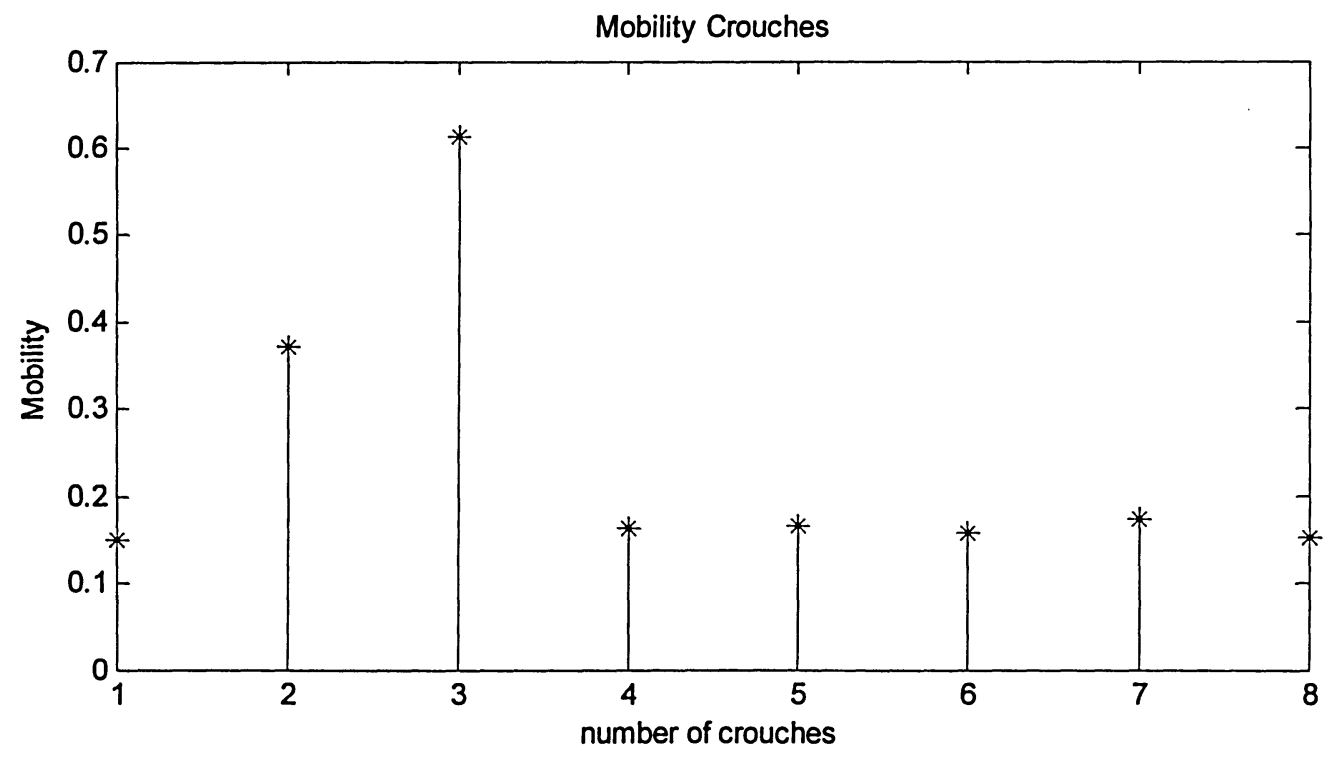

Figure 26: The figure shows the mobility calculated for eight crouches. The crouches were taken from three subjects at random times into the virtual environment. Crouch number 1 is Subject A, crouches 2 and 3 are Subject B, and crouches 4 through 8 are Subject C. 


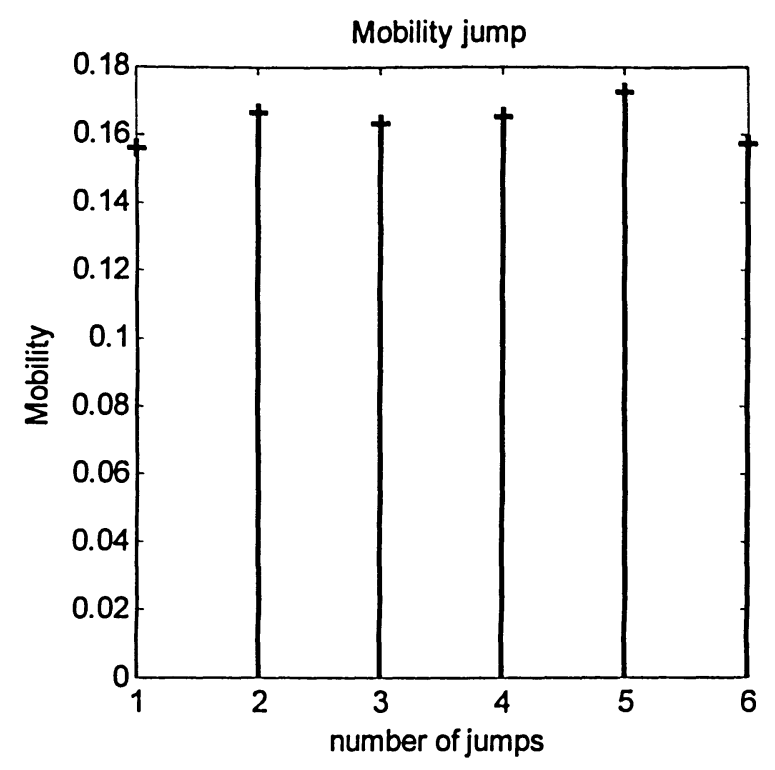

Figure 27: The figure shows the mobility calculated for six jumps. The jumps were taken from three subjects at random times into the virtual environment. Jump number 1 is Subject $B$, jump number 2 is subject $C$, jumps 3 and 4 are Subject $D$, and jumps 5 and 6 are Subject $A$.

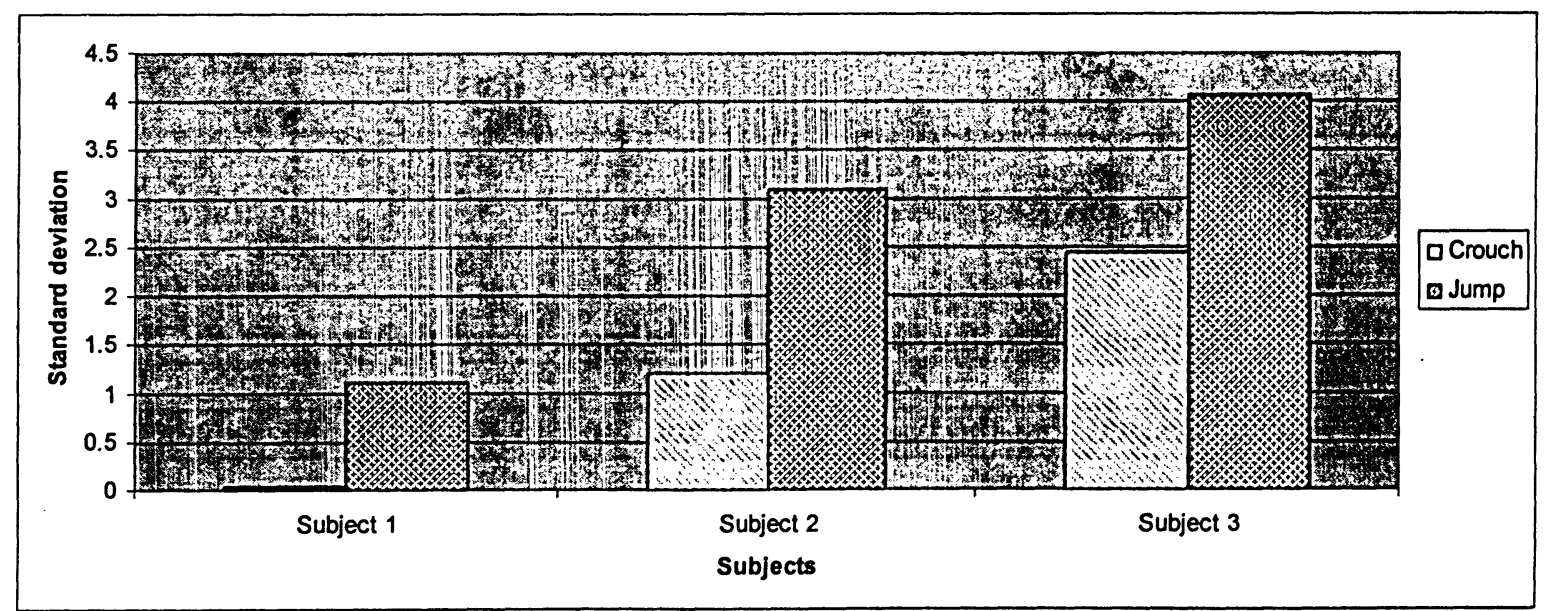

Figure 28: The standard deviation for both crouches and jumps for different subjects. 
To examine the effect of learning on EMG in the virtual environment, form factor in jumps (only jumps are considered here, as the previous results show jumps have more consistent form factor and mobility even for different subjects) were calculated from a session early in the training period and later in the training period. Contrary to previous findings, which showed that form factor decreased in the post sessions for balance board [table 1], in our case, the form factor increased for jumps in the later trials in the virtual environment.

Table 3: Table shows the form factor in the EMG signals that show jumps in the beginning of the training (session two, week one) and at the end of the training (session eight, week three).

\begin{tabular}{|r|r|r|}
\hline \multicolumn{1}{|c|}{ Subject } & Form Factor (session two) & Form Factor (session eight) \\
\hline 1 & 2.0909 & 4.1195 \\
\hline 2 & 3.6593 & 3.6913 \\
\hline 3 & 1.7268 & 2.7336 \\
\hline 4 & 2.8625 & 5.9475 \\
\hline
\end{tabular}

These findings are different from the data from the balance board (where post FF showed a decrease). It is hypothesized that the balance board and tandem Romberg data calculations for the form factor agree with the literature because the subject is in the same condition in both cases. However, the jumps in the VR (Table 3) are not the same jumps in session two and eight. For example, the jumps recorded in session eight likely took place at a different time and location in the VR than the jumps in session two. The sample size is also very small (one jump per subject) and the position and location of jump itself (for the same subject) is variable. Also, 
the response of the muscle might be different when a jump is followed by another jump or when a jump is followed by simple lateral movement or another crouch. This could explain why the FF during the VR training did not show a similar trend as in balance board and tandem Romberg data. These data suggest interesting areas for further experiments. The analysis results show that the Form Factor and Mobility can provide a standardized measure for progress in training as long as all the parameters are known and controlled.

\subsection{Head Movements}

Since the user uses head movements to guide the avatar in the VE, this requirement was hypothesized to encourage more head movements in everyday situations. The more the users move their head, the more they become aware of their surroundings. After the head movements were obtained and recorded in LabView, they were processed using MATLAB. A program was written to find the maximum angle in the left and the right direction. The following tables show the maximum head movements in the left and right direction for the nine users in an early session and a later session.

Table 4: Table shows the maximum and minimum displacement of head towards the left and right direction during the experiment in the second session.

\begin{tabular}{|r|r|r|}
\hline Subject & Right (degrees) & Left (degrees) \\
\hline 1 & 47 & 38 \\
\hline 2 & 67 & 85 \\
\hline 3 & 59 & 75 \\
\hline 4 & 88 & 86 \\
\hline 5 & 65 & 87 \\
\hline 6 & 66 & 88 \\
\hline 7 & 64 & 79 \\
\hline 8 & 31 & 76 \\
\hline 9 & 58 & 87 \\
\hline
\end{tabular}


Table 5: Table shows the maximum and minimum displacement of head towards the left and right direction during the experiment in the eighth session.

\begin{tabular}{|r|r|r|}
\hline Subject & Right (degrees) & \multicolumn{2}{|c|}{ Left (degrees) } \\
\hline 1 & 38 & 61 \\
\hline 2 & 63 & 85 \\
\hline 3 & 70 & 86 \\
\hline 4 & 26 & 72 \\
\hline 5 & 64 & 63 \\
\hline 6 & 78 & 88 \\
\hline 7 & 71 & 77 \\
\hline 8 & 70 & 63 \\
\hline 9 & 86 & 88 \\
\hline
\end{tabular}

From the tables above, it can be seen that for almost all the subjects the maximum head movements to the left and right are quite large (ranging from 26 to 88 degrees) at both the beginning and the end of the experiment. The head movements could lead to more awareness of the environment and better classification of the targets and obstacles.

Body and head movements coupled with gaze shifts as discussed earlier could help in alleviating the symptoms of vestibular dysfunction. The environment requires active gaze shifts from the users to identify potential pathways, obstacles and targets in the virtual reality and the game performance is based on how well they are able to locate and respond to those visual stimuli. The continuously changing visual field in the virtual environment and the appearance and disappearance of targets and obstacles, which in turn require quick response from the user, induce gaze shifts and hence could be used towards vestibular rehabilitation. The future applications of this experiment include the role of gaze shifts during training in improving balance responses. Ultimately, the impact of this training on elderly adults and adults with 
vestibular impairment will be tested. Even though this experiment was not conducted on these specific populations at this point, it provides preliminary support for using a cost effective VE in balance rehabilitation. 


\section{Chapter 5}

\section{Proposed Model and Simulations}

\subsection{Proposed Model}

It has been well established that visual cues are the most dominant cues in postural stabilization and they attenuate self-generated body sway by 50 percent $[41,42,43]$. In our day-to-day activities, we are moving in the environment, and the environment is stable for the most part. However, to study sensory integration, the experiments are often done in moving visual fields, where the subject is stationary and the visual field moves around him or her. This helps the researchers understand the response of an individual to the visual stimulus and to a larger extent is thought to be mimicking the response in the real world.

Our experiment was conducted in a virtual environment in which the visual cues are most dominant; however, the other inputs are also present. The vestibular, proprioceptive and somatosensory cues are self generated. In the experiment, the response of the visual stimulus was seen in terms of EMG signals taken from the soleus muscle of the subjects, and the head movements that the subject generated in the virtual environment.

The purpose of the proposed model is to predict the EMG response in a postural muscle of an individual in a virtual environment. Since only one muscle was used, the objective is simply to correlate the activation pattern of the response (indicating amount of body movement) to the 
dynamic characteristics of the visual display with an emphasis on its context or meaning to the user. The goal of the model is to characterize the EMG responses at different stages of training, so that individual progress can be quantified against a standard. The block diagram of the model is shown in Figure 29, and is described in detail below. It is acknowledged that there are various other ways to simulate the results, and numerous similar models exist in the literature $[31,32,21$, $63,64,65]$. This model is a simple yet accurate attempt to address the observed activation of the postural muscle in response to visual input with reference to responses stored in memory.

In different postural models in the literature, the input signal could be as complex as a combination of inputs from different sensors (as in Figure 1), or the input signal could be as simple as a combination of a visual and non-visual signal [71]. The signals may be corrupted by noise to account for malfunction of the sensors due to age or disease. Every sensor has its own transfer function to filter the input signal. The transfer function can be as simple as a low pass/high pass filter or simply a factor of 1 based on the experiment and the environment. After passing through respective sensor transfer functions, the input signals are then usually weighted based on thresholds and fixed gains selected by the researcher. The final response of the model could range from eye movements, or postural sway, to subjective vertical etc.

The simplified model is an attempt for effectively correlating the observed visual input with a stored pattern in memory, and assigning the appropriate output response multiplied by a scaling factor. The visual signal could be corrupted with noise to indicate any disease of visual system or to represent poor resolution or glare on the screen. For simplicity, the fixed gain is assumed to be 
1 as the only external input is a visual signal which is also the dominant signal. The output response is measured in terms of EMG.

As mentioned earlier, EMG signals are of importance to study the postural response; an attempt in the model is made to simulate similar responses as in the actual experiment. The input to the model is the visual signal that the user sees in the virtual environment. The signal is then compared to the database stored in memory to initiate an appropriate response. It is assumed that the data is stored in memory when the subject undergoes the tutorial before the first training session. He or she then identifies the targets, rings, obstacles, rails etc. in the environment and stores the correct response based on the prompt by the instructor in the demo.

The block diagram is shown in Figure 29. The input to the model is the visual signal. The other input signals (proprioceptive, somatosensory, cutaneous and vestibular) were self generated in the VR training environment, and therefore are not included in this analysis. The other signals can be included in the future to extend the model. Usually in orientation models in the literature, the input signals are weighted based on the accuracy of the signals or as determined by the researcher in the experiment (see Figure 9). Since there is only one input to the model, it is weighted $100 \%$. The model is unique from the other models in the literature as this model incorporates the memory component. It is assumed that the user had some prior knowledge of the obstacles, targets, rails etc. in the VE, from the tutorial in the first session. This enables users to classify those "events" (referring to different situations in the VE: in the model the event inputs refer to targets, take off ramps, a series of crouches, and a launch pad ahead of air borne rings, see Figure 30). The input signal is then compared with the database stored in the memory. A 
desired response (jump, crouch, lateral movement etc.) is generated after cross-correlating the significant events in memory with the visual signal. The cross correlation is done in MATLAB with a simple cross-correlation algorithm. In the future, if more events are stored in the model's memory database, advanced algorithms might be needed to classify and recognize the different events.

After classifying the event based on the cross correlation coefficient, the desired EMG response is generated in the model. For example, we know from the experiment that jumps have higher magnitude than the crouches, or the response when hitting the targets is different from random lateral movement, so a response is generated based on the classification (see equation 7). The individual gain W adjusts the amplitude of the EMG signals for different subjects (see equation 8). Notice that there is no feedback loop in the simplified model as the user/subject progresses in the virtual environment and does not encounter the same scene unless he or she repeats the course. There is some auditory feedback when a target is struck or the user is on the wrong path, but it is not considered in the simplified model. This feedback is the basis for the learning that the subject undergoes over the sessions, and is involved in better choices and options in the subsequent trials. This is reflected in updating the motor memory for subsequent sessions. 


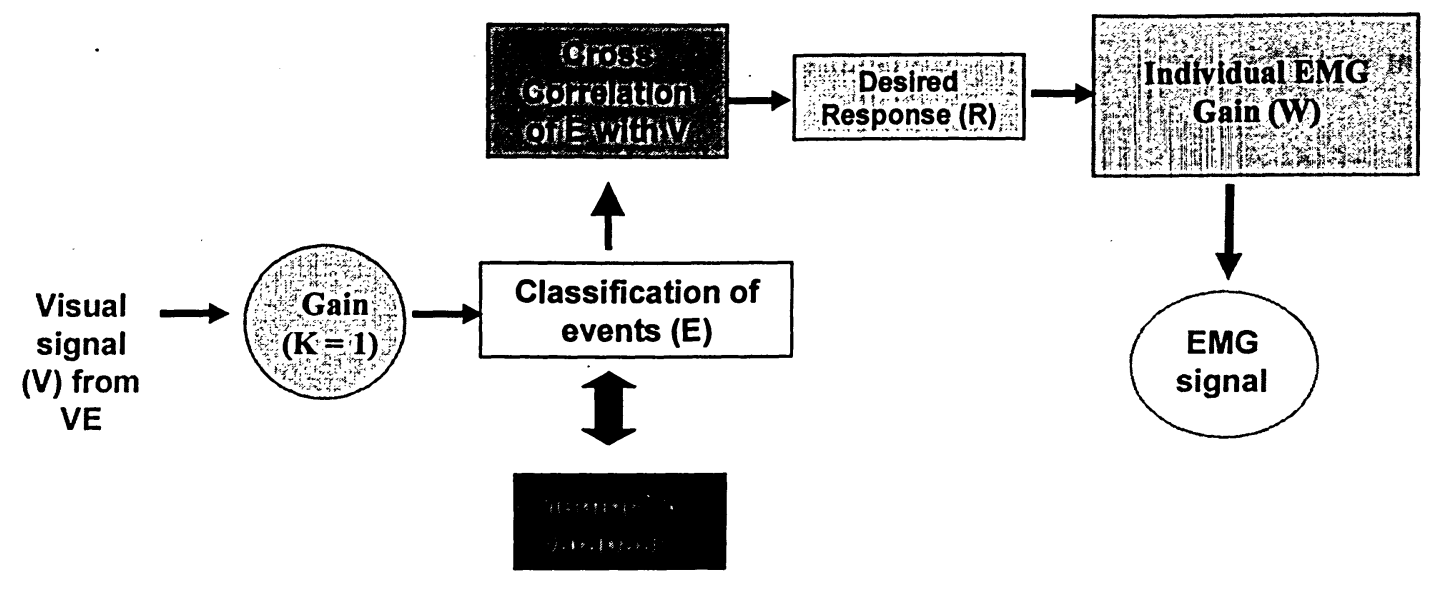

Figure 29: A simplified postural model for the virtual environment training showing the EMG response. The input to the model is the visual signal that the user sees in the virtual environment. The database stored in memory is used to classify significant events (targets, obstacles, rings, launch pads etc). A desired response (jump, crouch, lateral movement etc.) is generated after cross-correlating the significant events with the visual signal. The individual gain $\mathrm{W}$, a fixed gain for every individual, scales the simulated results to match the experimental parameters.

\subsection{Simulations}

The visual input that requires the subject to move laterally, jump, crouch, duck from obstacles, hit the targets etc. must be characterized as a signal in order to test the model. The visual input was characterized by the speed at which the image of a significant object moved across the retina. Figure 30 shows the spatial location of an object of interest as a function of time (each course in the virtual environment was roughly 5-6 minutes, so same time scale is used (400 seconds)), where the object of interest is defined as a target to strike or to avoid, i.e. the context or meaning of the image is obtained from memory leading to appropriate action. We characterized the series of targets to be struck by the user's hand movements on the sides on the rails as a sine wave input (targets are arranged starting from the bottom to the top and back down to the bottom). An example is shown as the first event in Figure 30. The ramp represents the 
take-off ramps, which launch the avatar into the air as a function of the speed of vertical head movement; the higher the jump, the more tricks can be performed for a higher score. This is shown as the second event in Figure 30. The series of pulses is a series of crouches to avoid obstacles. Finally the fourth event in Figure 30 is a step input representing the launch pad ahead of the airborne rings, and immediately superimposed on it is a sine wave, which represents the approach of the first ring. It is truncated at the point that the avatar passes the ring. It is acknowledged that this signal is highly simplified; it represents a cognitive signal reflecting the meaning of the image combined with the spatial motion properties, rather than a direct retinal map of the visual input.

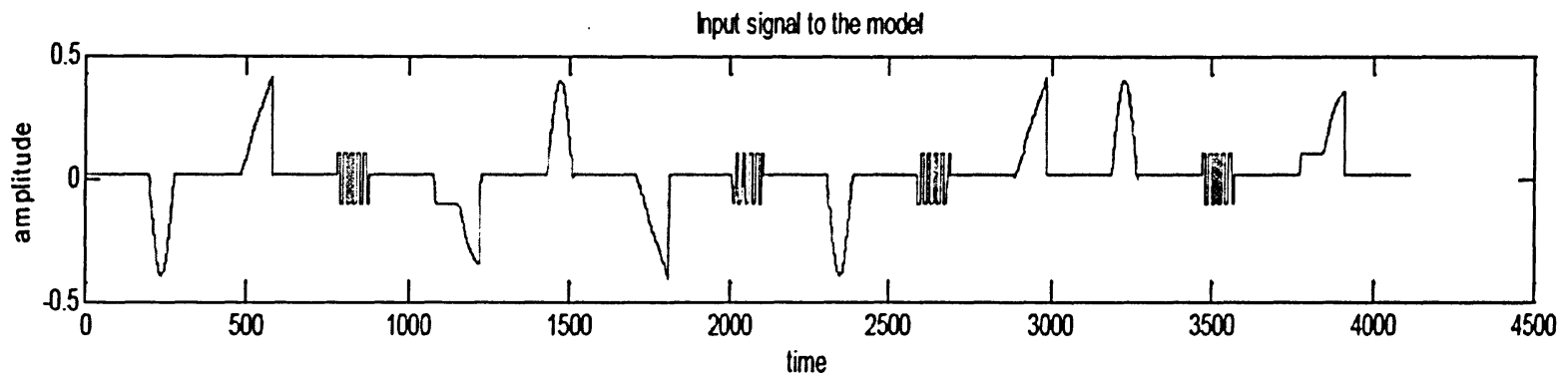

Figure 30: Figure shows the inputs to the visual system. The sine wave represents the targets that are arranged starting from the bottom to the top and back down to the bottom. The ramp represents the take-off ramps. The series of pulses is a series of crouches to avoid obstacles. Finally the fourth event is a step input representing the launch pad ahead of the airborne rings, and immediately superimposed on it is a sine wave, which represents the approach of the first ring. It is truncated at the point that the avatar passes the ring.

The database in memory consists of different events: the targets, the take-off ramps, series of crouches and launch pads. The database could be expanded to include more significant events as required by the experimenter. These events are classified from the signal based on memory and 
then cross-correlated with the input signal to generate a desired response. The cross-correlation is done as per equation (6), where $f$ and $g$ are discrete functions.

$$
(f \star g)_{i} \stackrel{\text { def }}{=} \sum_{j} f_{j}^{*} g_{i+j}
$$

(Cross-correlation is a measure of similarity of two signals, commonly used to find features in an unknown signal by comparing it to a known signal.)

We know from the experiment that an obstacle on the road requires a subject to dodge, a low obstacle on the rails initiates a jump, and a high obstacle initiates a crouch. Similarly, the ramp initiates a higher and more demanding response as seen in terms of the EMG of the subject's muscle, and navigating on the rails and on the course in the virtual environment induces appropriate lateral movement in the subject. So the desired responses are generated as sinusoidal signals in the model. The generic form of such a response is shown in equation (7):

$$
h(t)=a^{*} \sin \left(\omega_{1} t\right)+b^{*} \sin \left(\omega_{2} t\right)+c^{*} \sin \left(\omega_{3} t\right)
$$

where $\omega_{i}$ 's are the frequencies associated with each event, and $a, b, c$ are scaling factors for the amplitudes. Once the desired response is generated based on the significant event, it can be modified (amplitude and frequency) with individual EMG gain W, 


$$
w=u^{*} e^{j \omega_{0} t}
$$

where $u$ is the scaled amplitude and and $\omega_{0}$ is the scaled frequency.

The final output of the model is shown in Figure 31. Figure 32 shows the actual response of the subject to the visual input as recorded in the experiment by the experimenter (a note was made for the same or similar event).

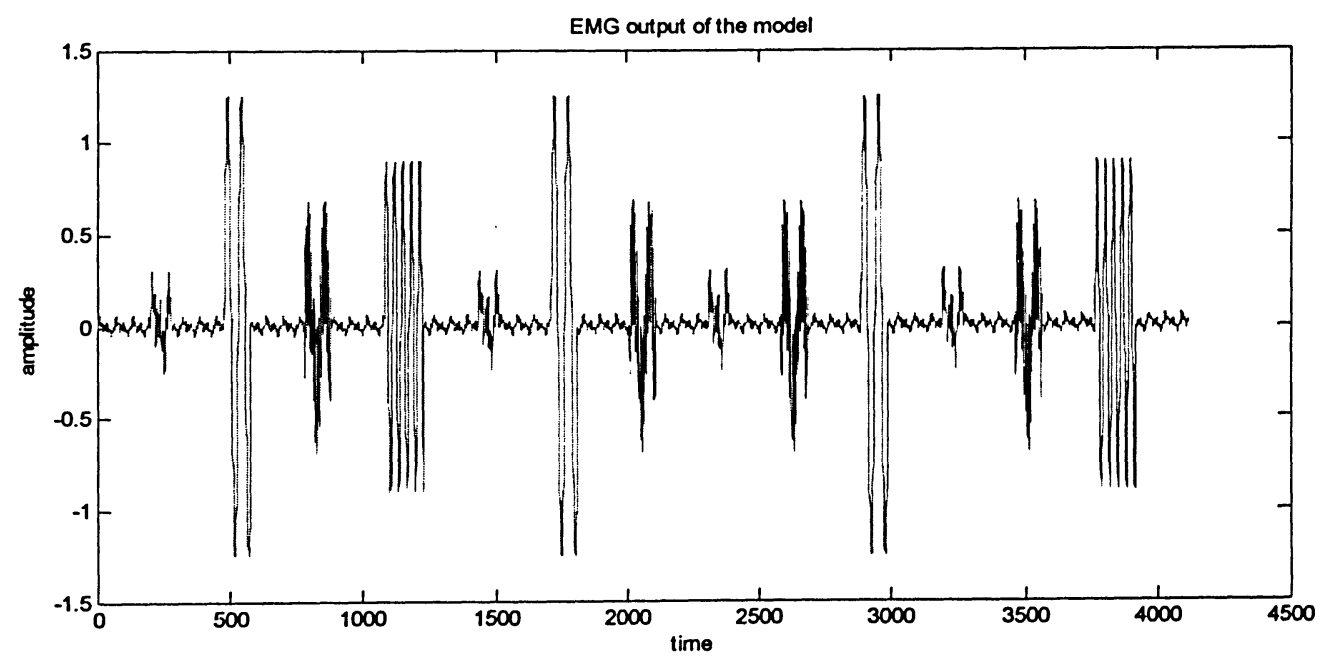

Figure 31: The response of the model to the input signal (as shown in Figure 30). 


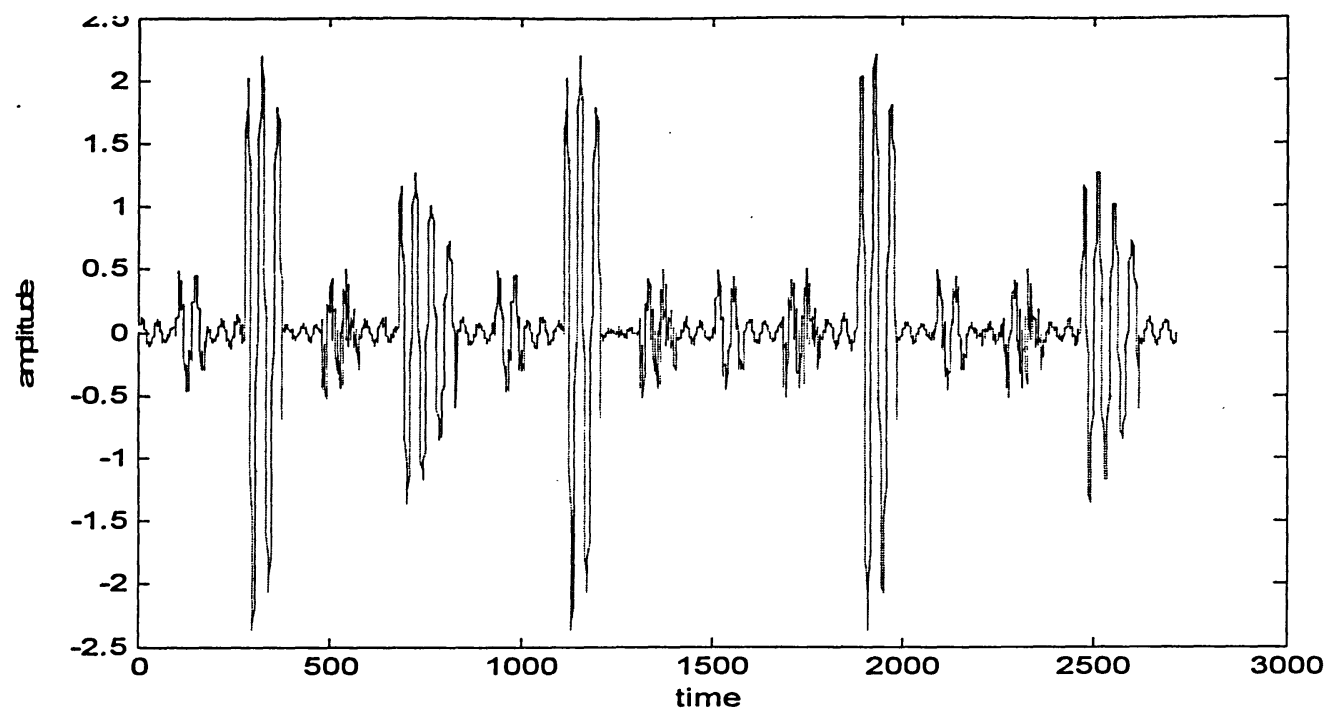

Figure 32: The actual response of a subject as recorded in his EMG signal to the input signal (as shown in Figure 30 ).

Figure 32 shows the response of a subject to the visual input. In the absence of any "events" (events corresponding to jumps, targets, rails, obstacles etc in the environment) there is some random muscle activation as seen in the EMG of the subject. However, the subject responds differently to the event inputs. For example, for the sine wave input, which is assumed to represent the targets on the sides on the rails in the virtual environment, the EMG shows different activity than the normal lateral movement. Similarly, for the ramp, which represents the take-off ramps, the response in the EMG is much higher in amplitude. For the pulse signal, that is a series of crouches, the response in the experimental EMG is very similar to that for the targets, but this EMG signal has a different frequency. For the input sine wave riding on a pulse that represents approach of the first ring, the EMG has higher amplitude than the other responses but a lower response than the take-off ramp. 


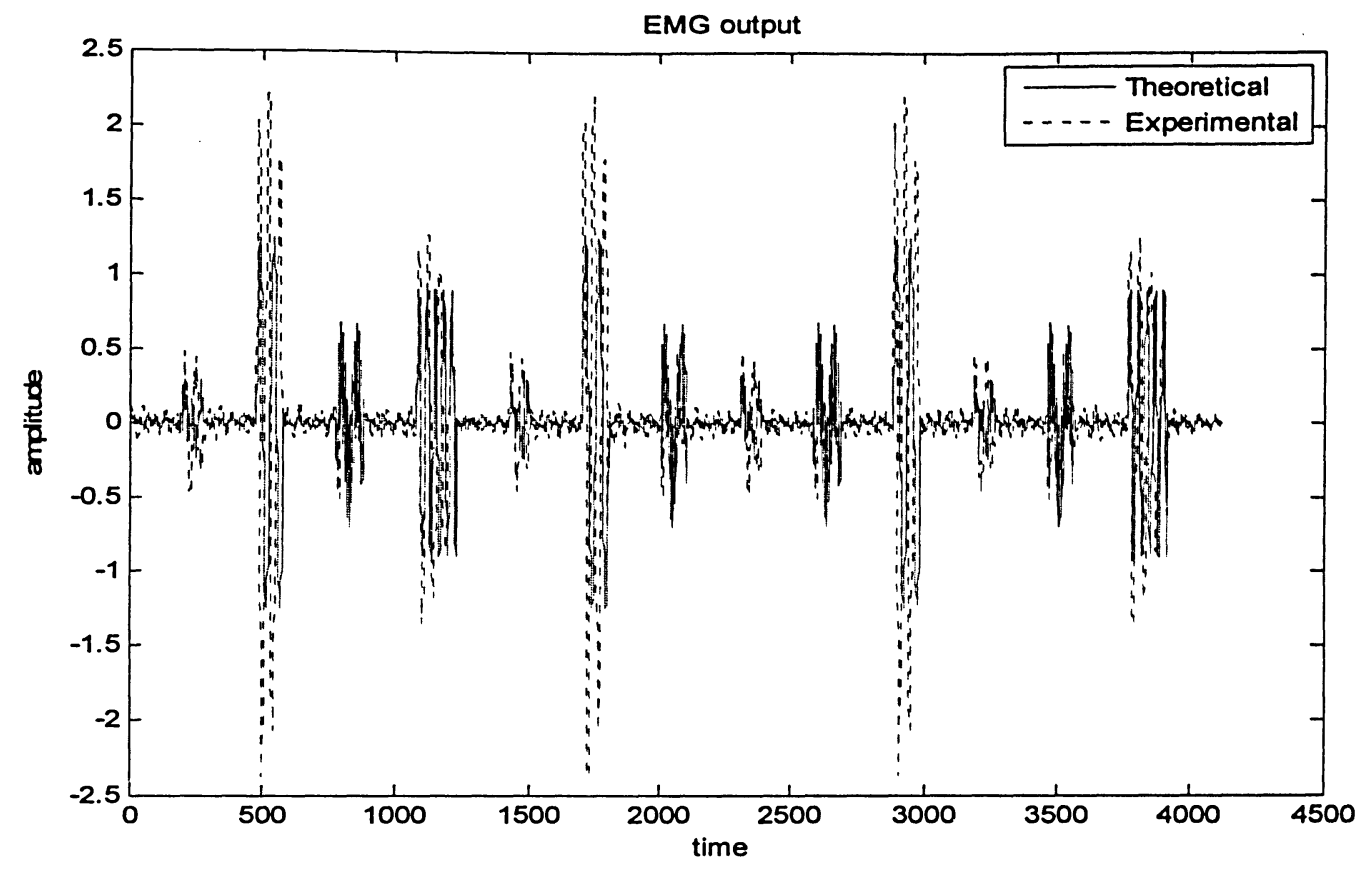

Figure 33: Results of the experimental EMG and theoretical EMG (predicted by the model) superimposed on each other. The blue graph shows the EMG predicted by the model, and the red graph shows the EMG recorded during the virtual environment immersion. Note that the results obtained from both the methods are very consistent.

The model response fits the experimental data well. The model recognizes the input and selects the desired response. This model serves as a basis for future applications and extensions.

\subsection{Model and Future Work}

In the future, the model could be extended to include feedback, vestibular and somatosensory inputs, modified signal to noise ratio of the sensory signals, and could be used to predict the motor learning response in EMG. The model could serve as an extension to existing models by taking into account memory and spatial information. Externally generated multi-sensory inputs are an important extension of the model. As discussed earlier, the visual inputs are dominant in spatial orientation and can be characterized by the frequency and position of the visual scene. Vestibular inputs can be measured by recording the head movements. Proprioceptive inputs can 
be obtained as the angles that the foot and the body make with respect to the surroundings and the body itself. Corrupted signals (representing illness or sensory impairments) can be simulated by adding random noise to the signals. Usually, all these inputs in the literature are measured in terms of angles so that the addition is facilitated in the model, but this usually requires specific experimental set-up to measure the angles precisely.

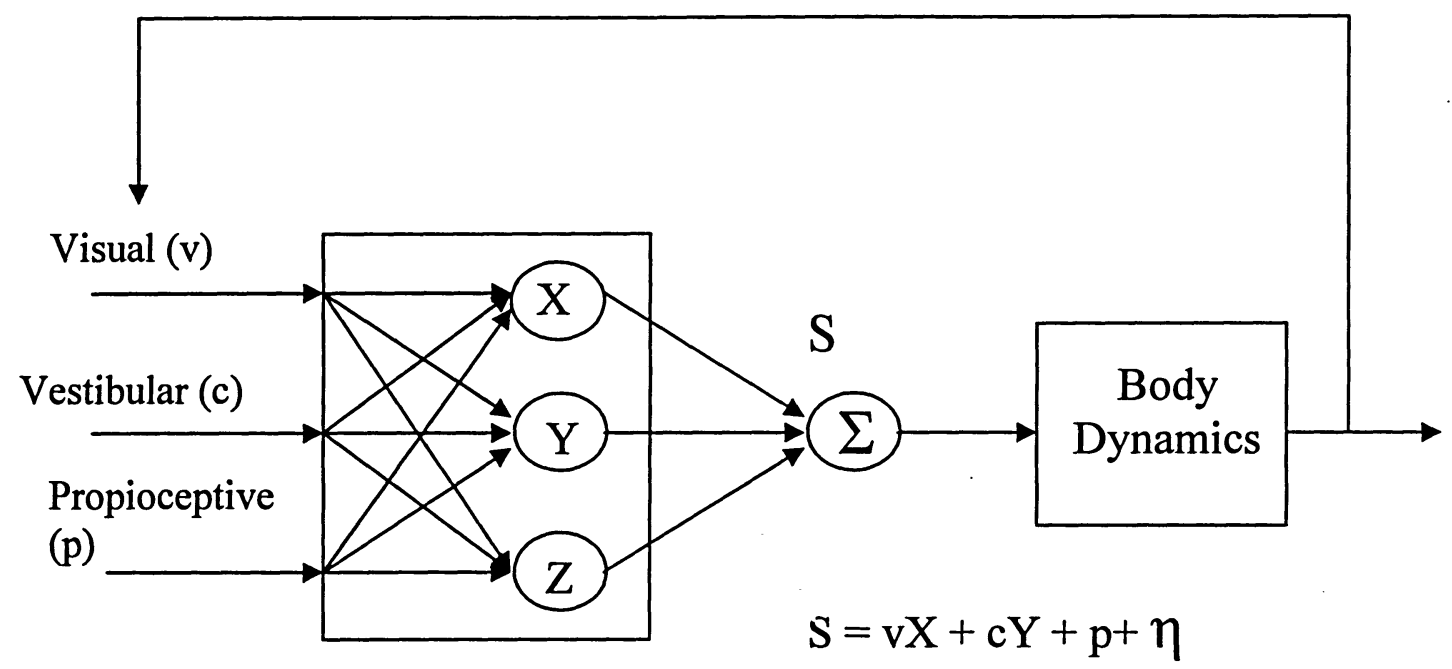

Figure 34: Comparator model of postural control: Sensory inputs are compared to each other for consistency, and a matrix of gains [v,c,p] is assigned accordingly. The weighted inputs are combined as $S$ which is the total sensed self-orientation with noise $\eta$ added. This results in a compensatory motor output through body dynamics. Feedback is provided through the senses.

The model could be expanded to explain multisensory interactions with feedback as shown in Figure 34. This figure is a general representation of an orientation model. The expanded model could be used to predict responses even when one of the sensors is giving a conflicting input or the information coming to the CNS from that particular sensor is not reliable. Under normal circumstances, vision is the dominant factor as we make judgments and responses based on what 
we "see". However, when there is a conflict or the visual information is limited (by fog or dim or no lights) we have to rely on vestibular or other cues that provide more consistent information. Additionally, we have seen from the literature that postural control is enhanced with greater reliance on non-visual inputs.

In certain cases where we know the environment or there is some prior knowledge of the environment in our memory, we tend to compare the response strategy to a previous experience we might have encountered. For example, it is easy for us to walk down the stairs of our basement even when there is no light because we have some spatial knowledge of that environment in our memory. So the model compares the coming inputs to a database of inputs built in the memory to execute a response. If it does not find any, we can extend the model to explain responses to novel inputs (without training). The CNS may assess reliability of different inputs using the SNR of the signals. The signal with highest SNR gets the most weight and the signal with lowest SNR gets the least weighting. It is interesting to mention here that we can not simply ignore a signal even when it is corrupted as the sensors will keep on picking up the signals unless they are physically blocked (for example the eyes will keep on picking up signals in a dim room unless a person shuts his or her eyes to block any signal). It is clear that many areas remain to be explored in the understanding of how the CNS recognizes and characterizes inputs and generates appropriate outputs.

The summation of the inputs could be treated as a linear or non-linear summation depending on the requirements of the experiment. Studies show that when visual inputs are doubled, the postural response saturates instead of doubling [21]. However, most researchers use a linear 
model for simplicity. Since all the models include the summation of the inputs, it is important that the inputs are measured in similar units. In most cases the inputs are measured in terms of angles, and the final output of the model is then position, velocity, or acceleration of the body joints, which in turn is a measure of dynamic posture control. To measure the effect of vestibular inputs on human posture, subjects with vestibular impairment are tested. Other sensory inputs can be disabled by adopting different measures. For example, the visual cues are blocked by conducting experiments in the dark, proprioceptive cues are disabled by adopting the 1:1 body sway referencing technique, and somatosensory cues are disabled by asking the subjects to stand on rubber foam or by applying ice to their feet and numbing the response. The transfer functions of the various sensors are documented in the literature [33-37]. The thresholds and the weights for the input signals are determined by the researcher to match the theoretical results with experimental findings. The optimal response of the models can be obtained by using optimization theory [34], implementing Kalman filter [31] to predict the postural response, or by using a simple PID (Proportional, Integral, Derivative) block [6]. The output of the models in the literature can range from EMG signals, to acceleration, velocity or position of the body joints, to subjective sensation of changes in vertical direction etc. The model could be extended to include visual vestibular interactions or to include memory and attention [32]. 


\section{Chapter 6}

\section{Conclusions}

Falls are a major healthcare challenge facing the elderly population. Falls can be caused by many factors such as vestibular impairment, disorientation, or by inadequate recovery reactions upon loss of balance. Other populations at risk of falls are those who work in physically challenging environments like athletes, nurses, firefighters and construction workers. Additionally, a lot of people also suffer from simulator sickness, which can include disorientation, in virtual environments, which range from advanced flight simulators for pilot training to home based entertainment.

In our research it is proposed that training in a virtual environment could help in improving balance, and could be used for vestibular rehabilitation, for alleviating simulator sickness symptoms, and for enhancing spatial knowledge and memory. Based on experimental results, a model was proposed that will help in understanding dynamic postural responses, which can be expanded in many ways, thereby shedding more light on strategies for balance and posture control in humans.

Experiments were conducted in a virtual environment to study the responses relating to balance and training, which was represented in the model as an updated database in memory. A low cost, off the shelf virtual reality environment was evaluated with respect to its effect on balance tests, 
head movements, EMGs, and game score improvement (reflecting improved reaction times and updated memory through learning the environment). Two levels of difficulty were used in the experiment, which was conducted in nine sessions over the course of three weeks. It was found that subjects felt very immersed in the environment and that user performance increased gradually in the environment. Head movements were required by the game consistently throughout the experiment period and between difficulty levels, and were recorded up to $88^{\circ}$ left and right. Balance test results indicated that the subjects were able to improve their performance on the balance tests after training. The environment also puts demands on users' cognitive and spatial skills, making it ideal for use in vestibular and motor rehabilitation. The EMG showed how the leg muscle behaved under unpredictable and challenging situations. By analyzing the EMG signals, it was possible to correlate the activity in the muscle with specific events in the training game. The model simulation results matched the experimental results very closely. The model could be extended to study multisensory interactions thereby shedding more light on real world responses and specific conditions such as motion sickness and disorientation.

The experiment and the training in virtual environment in particular were rated as enjoyable by all the subjects. There were some mild symptoms of simulator sickness in the beginning but the symptoms soon subsided as the training progressed. The experimental data shows that the visual inputs and the body motion were tolerated well by the healthy subjects; it is hypothesized that these findings provide a basis to conduct the experiment in elderly population and in people with vestibular impairment (with appropriate modifications). The VE chosen for this study is a challenging and enjoyable game with progressive difficulty levels. As an inexpensive solution 
that could be applied for home use, it has the advantage of allowing those concerned about their risk of falls to train themselves in a safe home environment.

As the research continues, the focus on establishing a home based rehabilitation capability for elderly and people with vestibular impairment will reduce the number of trips to a therapist and will keep them motivated to perform their exercises, subsequently leading to improved balance, reduced risk of falls, and a better quality of life. The usefulness of the work will also be seen in the development of testing protocols, which can predict or flag risks and problems, in the development of preventative strategies, and ultimately in the reduction of the number of falls experienced by the elderly or by people having sensory-motor impairment. 


\section{Appendix A}

\section{Questionnaire}

1. Age:

2. Sex: Male Female

3. Height:

4. Weight: Feet lbs Inches

5. Are you considered yourself in a "relatively good health" condition? YES NO

6. Are you active? YES NO Describe your activity.

7. Have you fallen more than once in the past year? YES NO

8. Do you feel dizzy or unsteady if you make sudden changes in movement such as bending down or quickly turning? YES NO

9. Do you have black-outs or seizures? YES NO

10. Have you experienced a stroke or other neurological problem that has affected your balance? YES NO

11. Do you experience numbness or loss of sensation in your legs and/or feet? YES NO

12. Are you inactive? (Answer yes if you do not participate in a regular form of exercise, such as walking or exercising 20-30 minutes at least three times a week.) YES NO

13. Do you feel unsteady when you are walking, climbing stairs? YES NO

14. Do you have difficulty sitting down or rising from a seated or lying position? YES NO

If you answered "yes" to one or more of the questions 7-14, then you could have a balance problem.

Source: www.BalanceandMobility.com 


\section{Appendix B}

\section{Simulator Sickness Questionnaire}

Please indicate the degree to which you are experiencing the following symptoms:

General discomfort

Fatigue

Headache

Eyestrain

Difficulty focusing

Increased salivation

Sweating

Nausea

Difficulty concentrating

Fullness of head ${ }^{1}$

Blurred vision

Dizzy (eyes open)

Dizzy (eyes closed)

Vertigo 2

Stomach awareness

Burping

$\begin{array}{cccc}\text { None } & \text { Slight } & \text { Moderate } & \text { Severe } \\ \text { None } & \text { Slight } & \text { Moderate } & \text { Severe } \\ \text { None } & \text { Slight } & \text { Moderate } & \text { Severe } \\ \text { None } & \text { Slight } & \text { Moderate } & \text { Severe } \\ \text { None } & \text { Slight } & \text { Moderate } & \text { Severe } \\ \text { None } & \text { Slight } & \text { Moderate } & \text { Severe } \\ \text { None } & \text { Slight } & \text { Moderate } & \text { Severe } \\ \text { None } & \text { Slight } & \text { Moderate } & \text { Severe } \\ \text { None } & \text { Slight } & \text { Moderate } & \text { Severe } \\ \text { None } & \text { Slight } & \text { Moderate } & \text { Severe } \\ \text { None } & \text { Slight } & \text { Moderate } & \text { Severe } \\ \text { None } & \text { Slight } & \text { Moderate } & \text { Severe } \\ \text { None } & \text { Slight } & \text { Moderate } & \text { Severe } \\ \text { None } & \text { Slight } & \text { Moderate } & \text { Severe } \\ \text { None } & \text { Slight } & \text { Moderate } & \text { Severe } \\ \text { None } & \text { Slight } & \text { Moderate } & \text { Severe }\end{array}$

1 Fullness of head refers to an awareness of pressure in the head.

2 Vertigo refers to a loss of orientation with respect to vertical or upright.

Source: Kennedy et al (1993) 


\title{
Appendix C
}

\section{Consent Form}

\section{Virtual Environment System Training for Improved Balance}

\author{
Research Ethics Board \\ Ryerson University
}

Before you give your consent to be a volunteer in this study, it is important that you read the following information and ask as many questions as necessary to be sure you understand what you will be asked to do.

\section{Investigators:}

Dr. Kristiina McConville : Ph.D., P.Eng., Associate Professor, Ryerson University Sumandeep Virk: M.A.Sc. Student (II year, Electrical Engineering, Ryerson University)

\section{Purpose of the Study:}

The study will evaluate the potential for this specific virtual reality training in improving balance.

\section{Description of the Study:}

You will be randomly assigned to the experimental group or control group. The subjects in the experimental group will undergo training, whereas the control subjects will only be doing the before, during and after balance tests, and will thus have fewer sessions.

The study is designed to train people to improve their balance. You will be trained in a virtual environment, in which you play a game on Sony's PlayStation II. This is a skateboard/hoverboard game in which you move and interact with a virtual environment through a cartoon character representing you in the game. There are two levels in the game that you are required to interact with. You will pass to level II if you beat the desired score in level I. In the real environment, you will stand on firm ground and will move your body, arms and head. This motion is detected by the Sony camera. You are also required to reach for targets with your hands and avoid obstacles by crouching or jumping, and your success rate will be recorded along with your speed of travel. You can control the speed of travel by crouching and standing. The eligibility criteria for the study are that subjects will be between the ages of 18-55, healthy, without any history of falls, or impairment that could affect balance as determined by the initial questionnaire. Twenty subjects will be recruited. 
On the first day, you will be asked to fill out a questionnaire, which will be used to determine your eligibility for the study. This will not take more than fifteen minutes. If you are eligible, you will continue on the same day. There will be three balance tests: In the first test, you will be asked to achieve and maintain balance on a balance board. The second test is a static posture test: the "tandem Romberg" test. In this you will stand heel to toe, with shoes off, head straight ahead, arms folded against chest. In the third test, you will be asked to stand on one foot with eyes closed. Your performance on these tests will be timed.

Then you will be trained in the virtual environment (VE) game. There will be two training periods of 15 minutes in each session. A rest break of 10 minutes will be provided between these two periods. In the second training period, based on your performance in the game, you will be moved to the next level. Otherwise, you play level I again.

In some virtual environments subjects have reported discomfort similar to motion sickness. This occurs more often when a head-mounted display is used. To reduce any chance of discomfort, you will be asked to complete a Simulator Sickness Questionnaire during the break and after the training.

Throughout the testing and training, electromyographic (EMG) signals from two muscles, head movement, and foot pressure distribution will be recorded. The EMG recording will involve adhesion of two pre-gelled electrodes. These electrodes passively record the electrical activity naturally present within your muscles and do not conduct any electrical current or voltage to you. The other recordings will all be electrically isolated from you (head movement is based on accelerometers and foot pressure is based on pressure exerted through your feet). Your training will be recorded on the Sony Playstation system, and additional video recording may also be performed during the training and balance testing for quantitative analysis.

You will be asked to come three times a week (about 1 hour each session), for three weeks to Ryerson University. The research will be conducted in a laboratory environment at Ryerson. The experimenter will work around your schedule for setting up the experimental dates, but three sessions per week with reasonable spacing are required. You will have to undergo the balance tests after the fourth session and again after the last training session.

\section{What is Experimental in this Study:}

The major aim of this study is to gather of information for the purpose of analysis. The experimental procedure of the study is to evaluate whether the training is an effective tool for improving balance. The hypothesis to be tested is that the virtual environment training will result in better balance strategies.

\section{Risks or Discomforts:}

The potential risks with the experiment are: 1 . loss of balance and falling, 2. electrical contact with instrumentation, and 3. simulator sickness, which is similar to motion sickness. The risks are considered minimal as 1 . only young healthy subjects will be used with minimal challenge to balance, and you will have tables around you to grab for support if you lose your balance during 
the posture testing, 2 . electrical contact with any power source is very unlikely, and 3 . the virtual environment is a visual display only (you will not be on a moving platform), and you will not be permitted to continue if you experience any simulator sickness symptoms. You are free to discontinue participation at any time.

\section{Benefits of the Study:}

This study will benefit a large amount of population: elderly, people who are at risk of falling, people who have fear of falling or have history of falling, and will also help to reduce the number of occupational falls. The data collected will give an insight into using virtual reality in treating balance problems and hence will help in improving balance and reducing falls. There are no benefits as such for the subjects who participate in the study.

\section{Confidentiality:}

No names of the participants will be disclosed in any publication. Subjects will be referred to by an assigned number during analysis and publication of results. The researchers will have the only access to data. It will be stored on Ryerson computers accessible by user ID and password authentication, and any paper copies will be locked in the researcher's office. The graduate student researchers will only have access to data for the period of their study. The supervisor (K. $\mathrm{McC}$ Conville will keep the data for 5 years after which it will be destroyed). The trials may be video-taped. These tapes will be used only for quantitative assessment and confirmation of performance during training and testing. Only the researchers (supervisor and graduate students (S.Virk and L. Kant) will have the access to these. The subjects will not be able to review or edit the tapes prior to any publication. The cartoon character recordings may be shown referencing subjects only by number code during presentations of the data. Video recordings of the actual subjects will never be shown to anyone other than the three stated researchers without separate written consent by the subject.

\section{Incentives to Participate:}

The participants will be paid an honorarium of $\$ 50$ at the completion of this study. If the entire study is not completed, no payment will be provided. If the study is discontinued due to injury or discomfort as determined by the researcher or subject, then payment will be prorated based on $\$ 5$ per session.

\section{Costs and/or Compensation for Participation:}

There are no costs (other than travel to and from the University) or compensation other than the incentive described above associated with the participation.

\section{Compensation for Injury:}

It is unlikely that participation in this project will result in harm to participants. If any complications arise, we will assist you in obtaining appropriate attention. We will provide transportation to home in the case of illness or to a medical facility in the case of injury. While 
every measure will be taken to avoid any possible injury or risk of injury, if any should occur, the researchers will refer the participant to any necessary medical care, such as the Occupational Health Clinic at Ryerson University, the local hospital or physician.

\section{Voluntary Nature of Participation:}

Participation in this study is voluntary. Your choice of whether or not to participate will not influence your future relations with Ryerson University. If you decide to participate, you are free to withdraw your consent and to stop your participation at any time.

At any particular point in the study, you may refuse to answer any particular question or stop participation altogether.

\section{Questions about the Study:}

If you have any questions about the research now, please ask. If you have questions later about the research, you may contact.

$$
\begin{aligned}
& \text { Kristiina McConville } \\
& \text { Ryerson University } \\
& \text { (416) 979-5000 X } 6085
\end{aligned}
$$

If you have questions regarding your rights as a human subject and participant in this study, you may contact the Ryerson University Research Ethics Board for information.

Research Ethics Board c/o Office of the Associate Vice President, Academic

Ryerson University

350 Victoria Street

Toronto, ON M5B 2K3

416-979-5042

\section{Agreement:}

Your signature below indicates that you have read the information in this agreement and have had a chance to ask any questions you have about the study. Your signature also indicates that you agree to be in the study and have been told that you can change your mind and withdraw your consent to participate at any time. You have been given a copy of this agreement.

You have been told that by signing this consent agreement you are not giving up any of your legal rights.

Name of Participant (please print)

Signature of Participant

Date 


\section{Agreement to be videotaped:}

Your signature below indicates that you agree to be videotaped during the study. These video recordings will be used for quantitative assessment by the researchers only, for example to validate the data obtained through head movement or muscle or foot pressure recordings. This agreement is voluntary and does not affect your participation in the study.

Name of Participant (please print)

Signature of Participant Date

Signature of Investigator

Date

\section{Agreement for videotape being shown:}

Your signature below indicates that you agree that videotapes taken of you during the study may be shown during research presentations or for educational purposes. This agreement is voluntary and does not affect your participation in the study.

Name of Participant (please print)

Signature of Participant

Date

Signature of Investigator

Date 


\section{Appendix D}

\section{Block Diagrams}

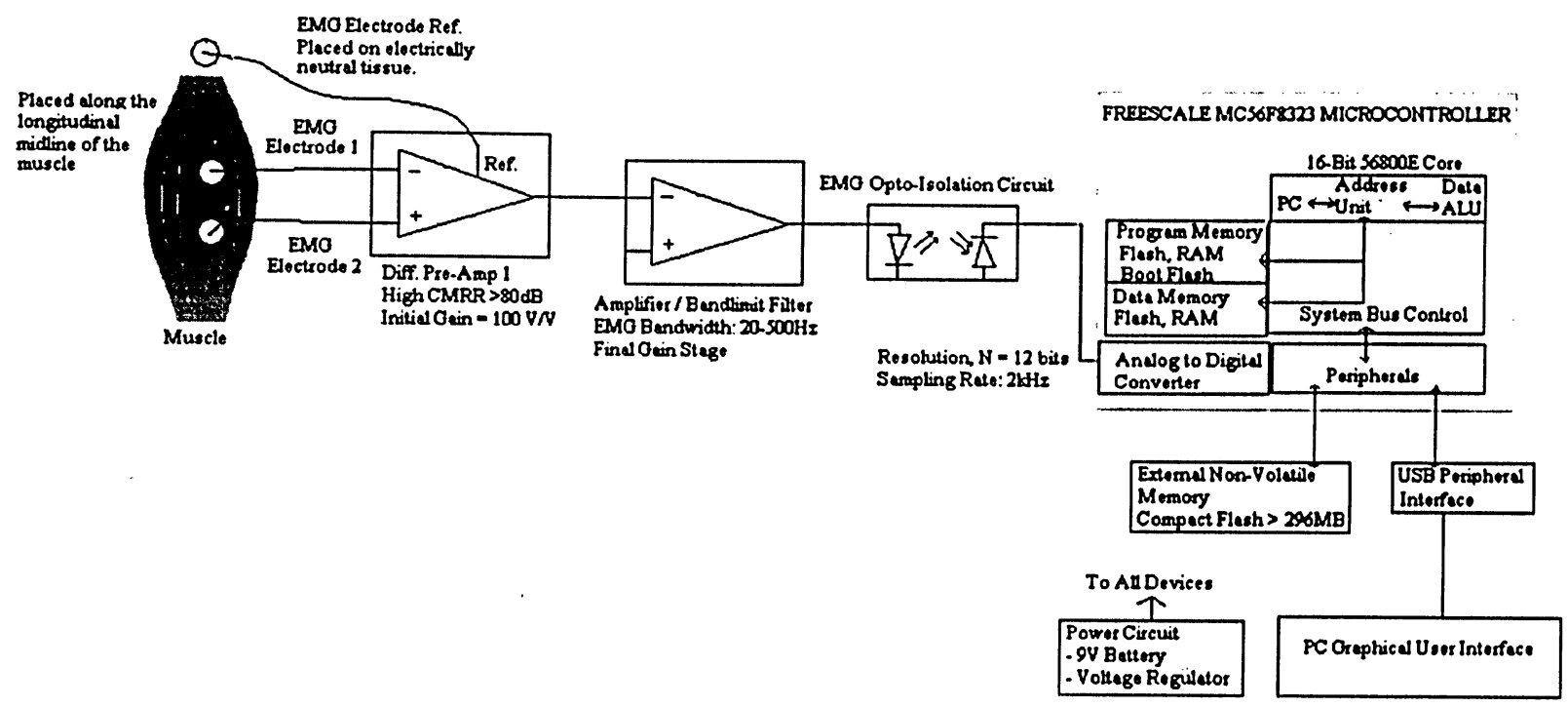

Figure D1: EMG system Block Diagram. Adapted from [69].

Head Movements Block Diagram

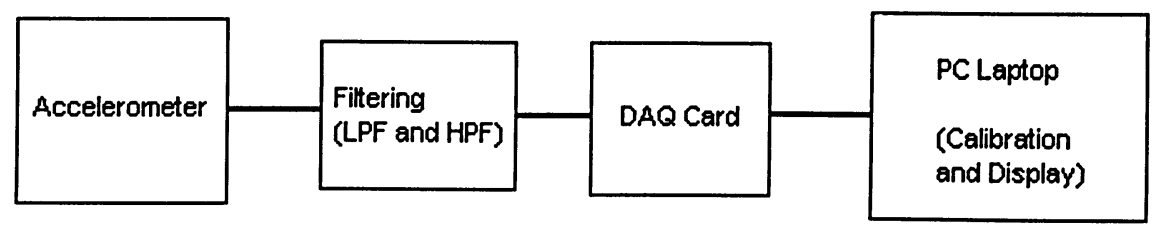

Figure D2: Block Diagram off Head movement system. Adapted from [70]. 


\section{Appendix E}

\section{Statistics}

\section{Balance Board Correlations and T-Tests}

\section{Paired Samples Correlations}

\begin{tabular}{|ll|r|r|r|}
\hline & $\mathrm{N}$ & \multicolumn{1}{|c|}{ Correlation } & \multicolumn{1}{c|}{ Sig. } \\
\hline TEST & BBPre \& BBPost & 9 & .688 & .041 \\
\hline CONT & BBPre \& BBPost & 6 & .934 & .006 \\
\hline
\end{tabular}

Paired Samples Statistics

\begin{tabular}{|rl|r|r|r|r|}
\hline & & Mean & N & Std. Deviation & \multicolumn{1}{c|}{$\begin{array}{c}\text { Std. Error } \\
\text { Mean }\end{array}$} \\
\hline TEST & BBPre & 71.000 & 9 & 36.6538 & 12.2179 \\
& BBPost & 150.778 & 9 & 46.0591 & 15.3530 \\
\hline CONT & BBPre & 104.917 & 6 & 82.9713 & 33.8729 \\
& BBPost & 90.917 & 6 & 77.3100 & 31.5617 \\
\hline
\end{tabular}

Paired Samples t-tests

\begin{tabular}{|l|l|r|r|r|r|r|}
\hline & \multicolumn{1}{|c|}{$\begin{array}{c}\text { Paired } \\
\text { Differences } \\
\text { Mean }\end{array}$} & $\begin{array}{c}\text { Paired } \\
\text { Differences } \\
\text { Std. } \\
\text { Deviation }\end{array}$ & \multicolumn{1}{c|}{$t$} & df & Sig. (2-tailed) \\
\hline TEST & $\begin{array}{l}\text { BBPre - } \\
\text { BBPost }\end{array}$ & -79.7778 & 33.8149 & -7.078 & 8 & 0.001 \\
\hline CONT & $\begin{array}{l}\text { BBPre - } \\
\text { BBPost }\end{array}$ & 14 & 29.6041 & 1.158 & 5 & 0.299 \\
\hline
\end{tabular}




\section{Tandem-Romberg Tests (TR) Correlations and T-Tests}

Paired Samples Correlations

\begin{tabular}{|ll|r|r|r|}
\hline & \multicolumn{1}{|c|}{ N } & \multicolumn{1}{c|}{ Correlation } & \multicolumn{1}{c|}{ Sig. } \\
\hline TEST & TRPre \& TRPost & 9 & -.353 & .352 \\
\hline & & & & \\
\hline CONT & TRPre \& TRPost & 6 & .971 & .001 \\
\hline
\end{tabular}

Paired Samples Statistics

\begin{tabular}{|c|c|c|c|c|c|}
\hline & & Mean & $\mathrm{N}$ & Std. Deviation & $\begin{array}{l}\text { Std. Error } \\
\text { Mean }\end{array}$ \\
\hline \multirow[t]{2}{*}{ TEST } & TRPre & 37.333 & 9 & 22.5333 & 7.5111 \\
\hline & TRPost & 106.444 & 9 & 28.8752 & 9.6251 \\
\hline \multirow[t]{2}{*}{ CONT } & TRPre & 64.528 & 6 & 48.9463 & 19.9822 \\
\hline & TRPost & 69.667 & 6 & 44.9240 & 18.3402 \\
\hline
\end{tabular}

Paired Samples t-tests

\begin{tabular}{|l|l|r|r|r|r|r|}
\hline & & $\begin{array}{c}\text { Paired } \\
\text { Differences Mean }\end{array}$ & $\begin{array}{c}\text { Paired Differences } \\
\text { Std. Deviation }\end{array}$ & \multicolumn{1}{c|}{$t$} & \multicolumn{1}{c|}{ df } & $\begin{array}{c}\text { Sig. (2- } \\
\text { tailed) }\end{array}$ \\
\hline TEST & TRPre - TRPost & -69.1111 & -42.4307 & 4.886 & 8 & 0.001 \\
\hline CONT & TRPre - TRPost & -5.1389 & 12.0326 & 1.046 & 5 & 0.343 \\
\hline
\end{tabular}




\section{One Leg Correlations and T-Tests}

Paired Samples Correlations

\begin{tabular}{|ll|r|r|r|}
\hline & N & \multicolumn{1}{|c|}{ Correlation } & \multicolumn{1}{c|}{ Sig. } \\
\hline TEST OLPre \& OLPost & 9 & .822 & .007 \\
\hline & & & & \\
\hline CONT & OLPre \& OLPost & & .460 & .359 \\
\hline
\end{tabular}

Paired Samples Statistics

\begin{tabular}{|c|c|c|c|c|c|}
\hline & & Mean & $\mathbf{N}$ & Std. Deviation & $\begin{array}{l}\text { Std. Error } \\
\text { Mean }\end{array}$ \\
\hline \multirow[t]{2}{*}{ TEST } & OLPre & 14.444 & 9 & 11.0918 & 3.6973 \\
\hline & OLPost & 27.111 & 9 & 15.4632 & 5.1544 \\
\hline \multirow[t]{2}{*}{ CONT } & OLPre & 26.361 & 6 & 16.8128 & 6.8638 \\
\hline & OLPOSt & 25.667 & 6 & 20.2180 & 8.2540 \\
\hline
\end{tabular}

Paired Samples t-tests

\begin{tabular}{|l|l|r|r|r|r|r|}
\hline & $\begin{array}{c}\text { Paired } \\
\text { Differences } \\
\text { Mean }\end{array}$ & $\begin{array}{c}\text { Paired } \\
\text { Differences } \\
\text { Std. Deviation }\end{array}$ & \multicolumn{1}{c|}{ T } & df & $\begin{array}{c}\text { Sig. (2- } \\
\text { tailed) }\end{array}$ \\
\hline TEST & OLPre - OL_Post & -12.6667 & 8.9582 & -4.242 & 8 & 0.003 \\
\hline CONT & OLPre - OLPost & 0.6944 & 19.4666 & 0.087 & 5 & 0.934 \\
\hline
\end{tabular}


Anova: Single Factor

\section{BALANCE BOARD}

SUMMARY

\begin{tabular}{crrrr}
\hline Groups & \multicolumn{1}{c}{ Count } & \multicolumn{1}{c}{ Sum } & Average & Variance \\
\hline Column 1 & 9 & 718 & 79.77778 & 1143.444 \\
Column 2 & 7 & -45 & -6.42857 & 1131.619 \\
\hline
\end{tabular}

COLUMN $1=$ TEST

COLUMN $2=$ CONTROL

ANOVA

\begin{tabular}{lcrrrrr}
\hline rce of Varie & SS & $d f$ & MS & $F$ & P-value & F crit \\
\hline Between C & 29261.67 & 1 & 29261.67 & 25.70474 & 0.000171 & 4.60011 \\
Within Gro & 15937.27 & 14 & 1138.376 & & & \\
Total & 45198.94 & 15 & & & F crit is < than F, so statistically significant \\
\hline
\end{tabular}

\section{TANDEM ROMBERG}

Anova: Single Factor

SUMMARY

\begin{tabular}{crrrr}
\hline Groups & \multicolumn{1}{c}{ Count } & \multicolumn{1}{c}{ Sum } & Average & Variance \\
\hline Column 1 & 9 & 622 & 69.11111 & 1800.361 \\
Column 2 & 7 & 31.83333 & 4.547619 & 123.0992 \\
\hline
\end{tabular}

ANOVA

\begin{tabular}{lcrrrrr}
\hline rce of Varie & $S S$ & $d f$ & \multicolumn{1}{c}{$M S$} & $F$ & $P$-value & $F$ crit \\
\hline Between C & 16413.25 & 1 & 16413.25 & 15.17589 & 0.001616 & 4.60011 \\
Within Gro & 15141.48 & 14 & 1081.535 & & & \\
Total & 31554.73 & 15 & & & & \\
\hline
\end{tabular}

\section{ONE LEG}

Anova: Single Factor

SUMMARY

\begin{tabular}{crrrr}
\hline Groups & \multicolumn{1}{c}{ Count } & \multicolumn{1}{c}{ Sum } & Average & Variance \\
\hline Column 1 & 9 & 68 & 7.555556 & 200.0278 \\
Column 2 & 7 & -38.1667 & -5.45238 & 463.1733 \\
\hline
\end{tabular}

ANOVA

\begin{tabular}{|c|c|c|c|c|c|c|}
\hline rce of Varie & SS & $d f$ & MS & $F$ & $P$-value & F crit \\
\hline Between G & 666.2502 & 1 & 666.2502 & 2.129926 & 0.16652 & 4.60011 \\
\hline Within Gro & 4379.262 & 14 & 312.8044 & & & \\
\hline
\end{tabular}

Within Gro 4379.262

$F$ crit is $>$ than $F$, so statistically not significant 


\section{Appendix F}

\section{Detailed Results of EMG Analysis of}

\section{Subjects}

In this appendix, detailed results of analysis of EMG signals obtained from jumps and crouches are shown. The purpose of this data was to help cluster jumps or crouches based on their form factor, mobility and standard deviation. The main reason to do that was to classify jumps and crouches from EMG signals based on these parameters and to look for these factors when looking for the subject's EMG in training. While some of the graphs suggest clustering, more data is required to determine the feasibility of any of these measures.

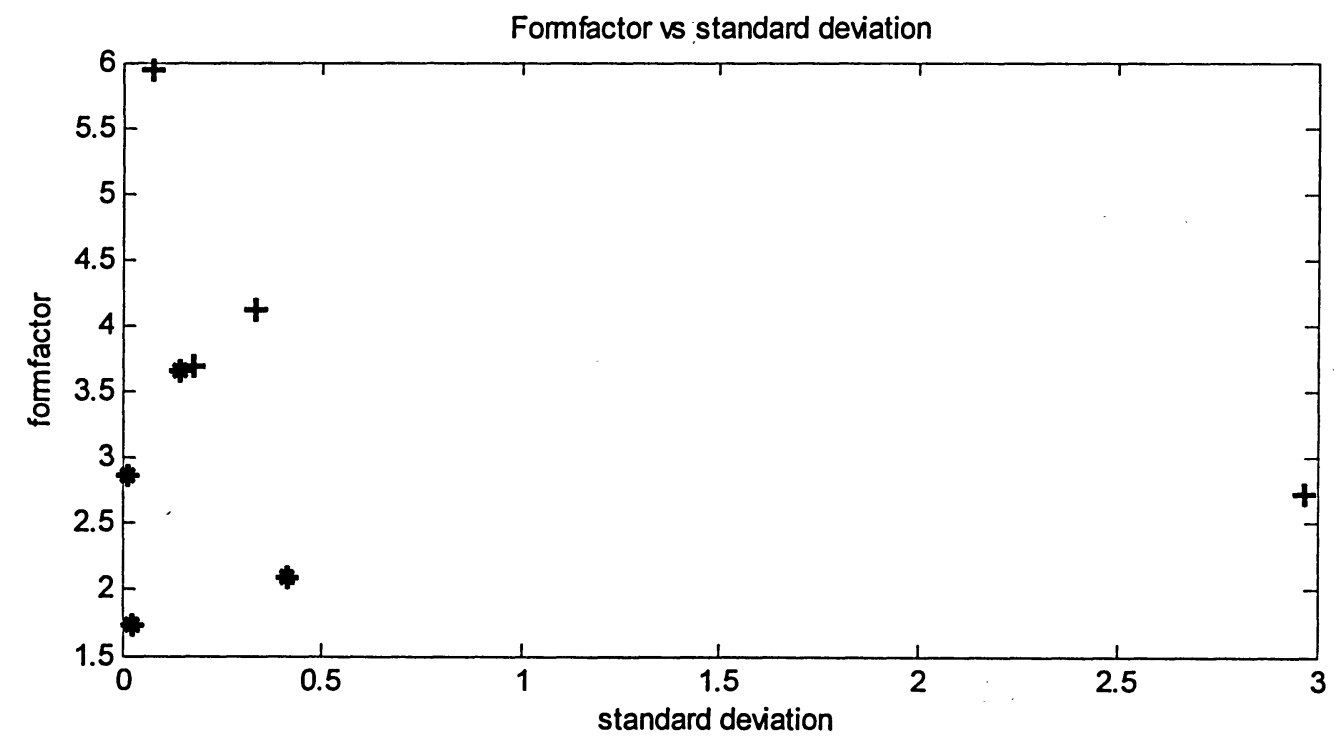

Figure F.1: The figure shows the form factor plotted against standard deviation for jumps in the early and later trials. The crosses are jumps from earlier trial and the stars are jumps from later trial in the training. 


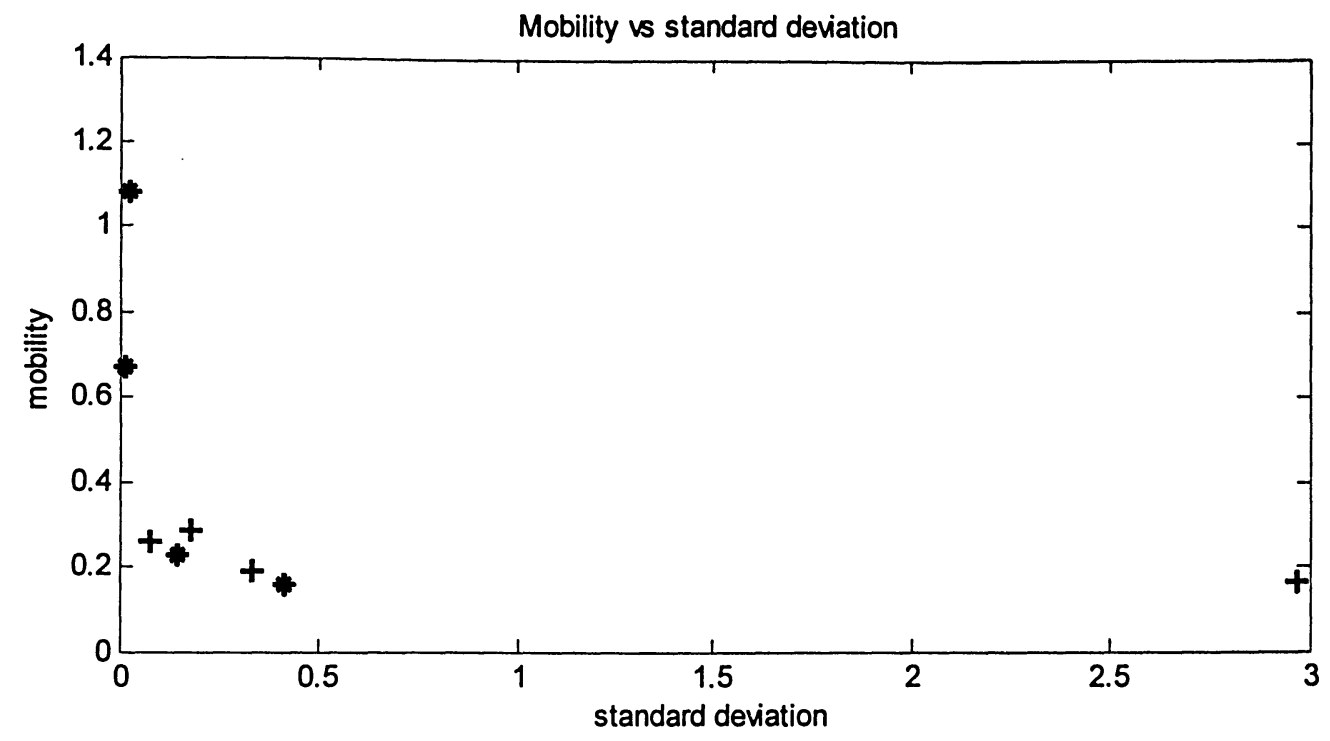

Figure F.2: The figure shows the mobility plotted against standard deviation. The crosses are jumps from earlier trial and the stars are jumps from later trial in the training.

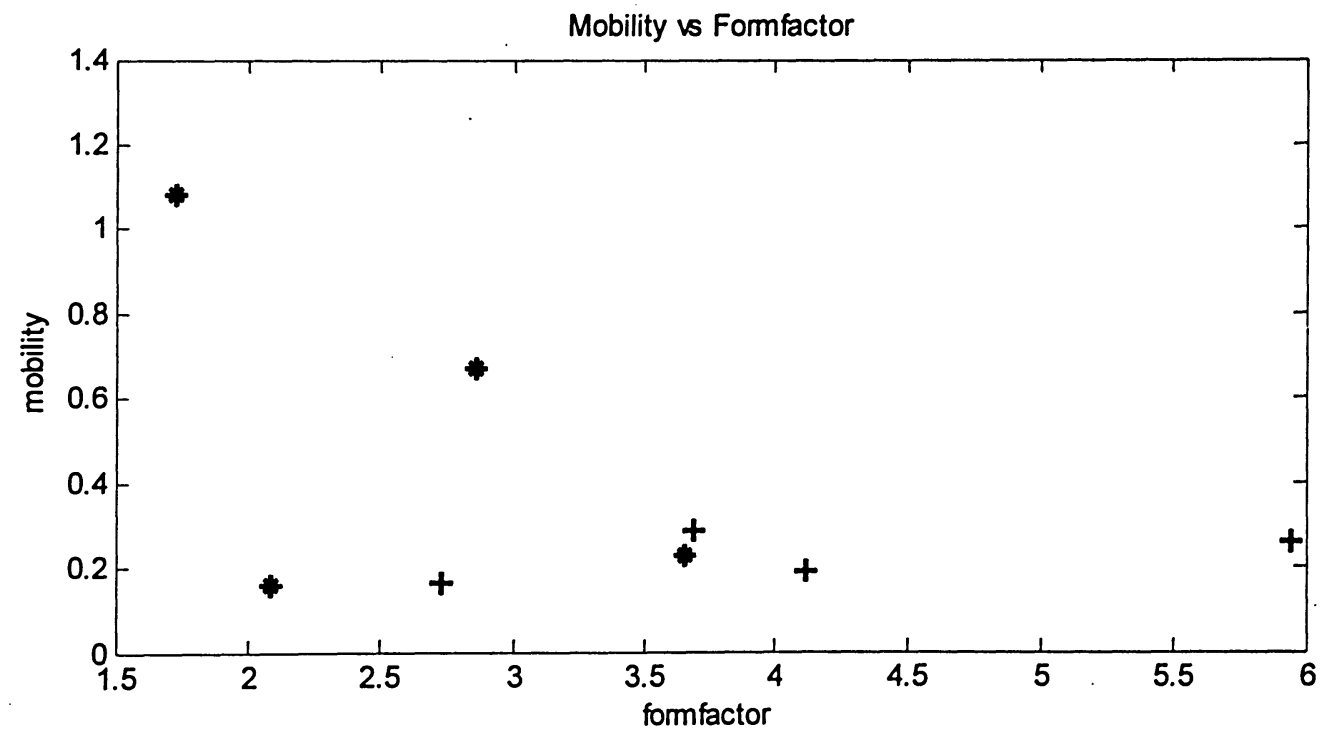

Figure F.3: The figure shows the mobility plotted against form factor. The crosses are jumps from earlier trial and the stars are jumps from later trial in the training. 


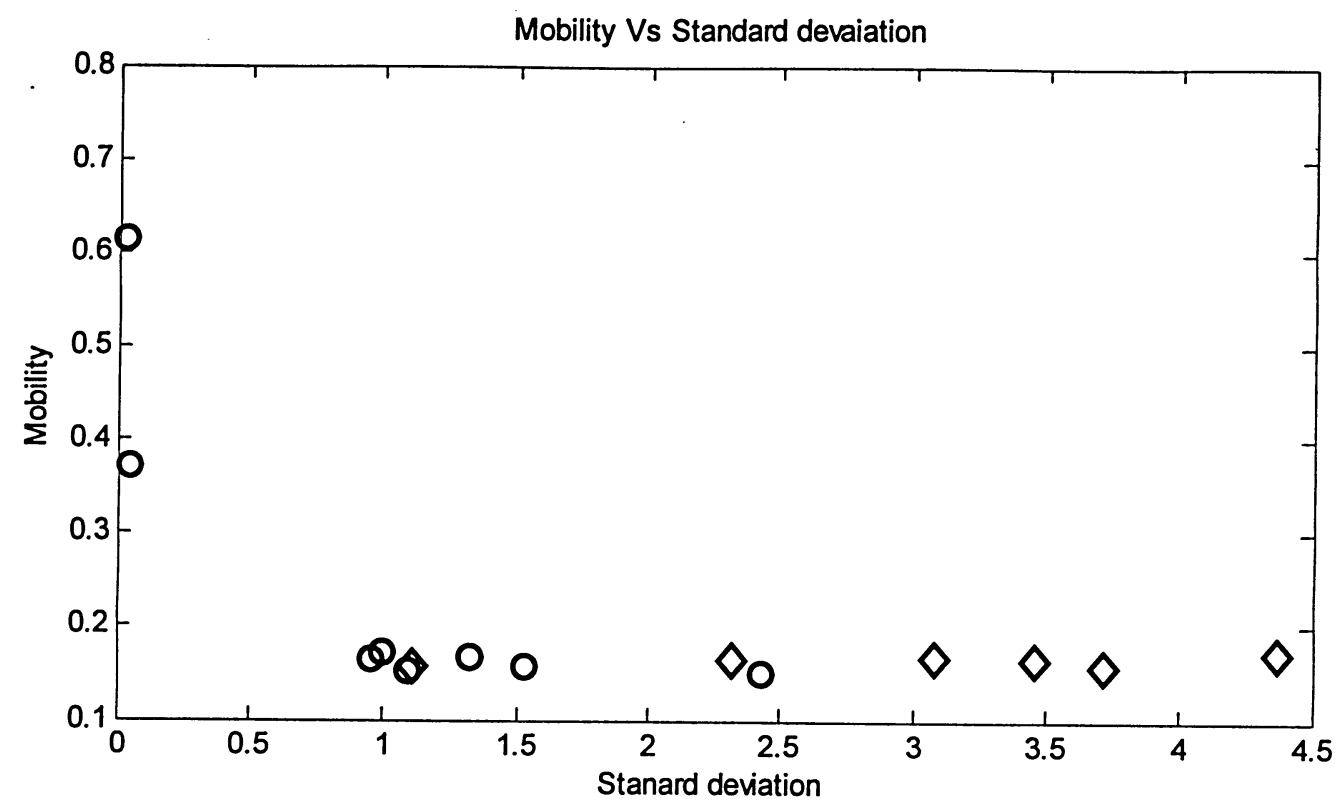

Figure F.4: The figure shows the mobility plotted against standard deviation for crouches and jumps. The diamonds are jumps and the circles are crouches. Both jumps and crouches are taken from random trials in the virtual environment.

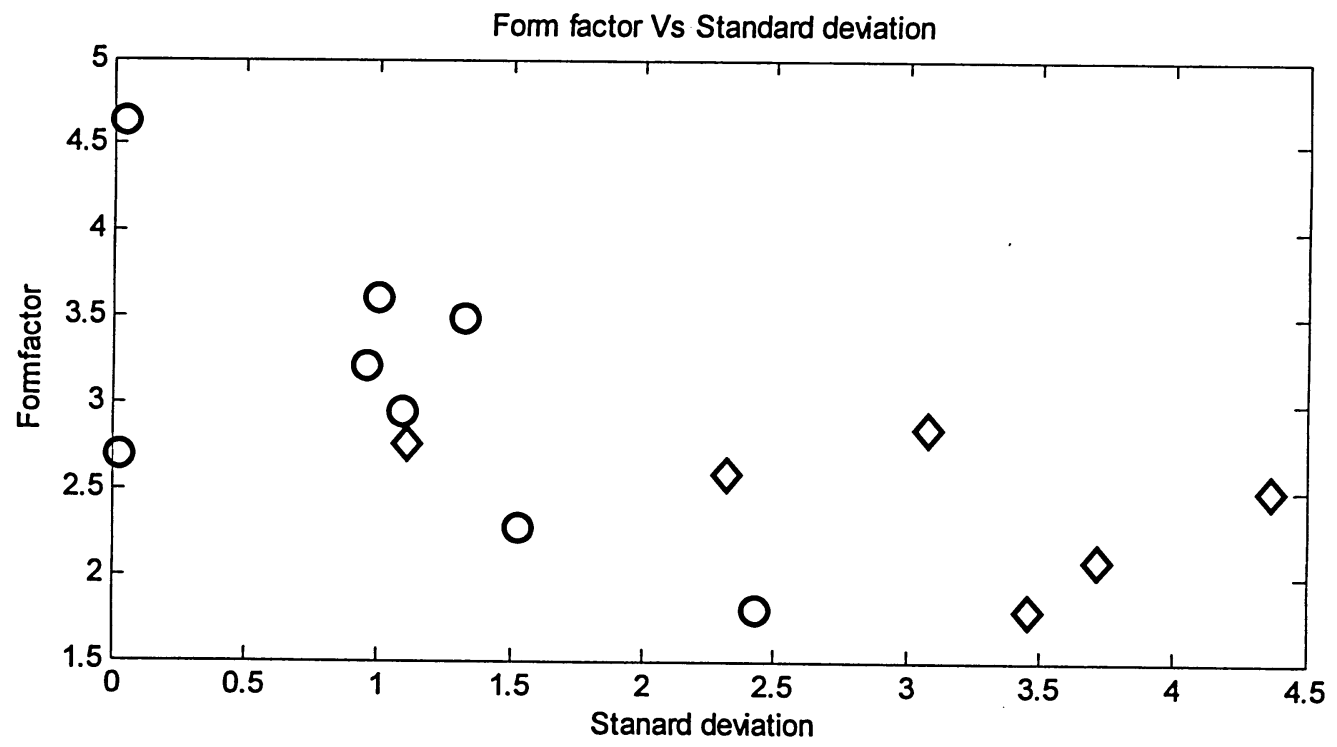

Figure F.5: The figure shows the form factor plotted against standard deviation for crouches and jumps. The diamonds are jumps and the circles are crouches. Both jumps and crouches are taken from random trials in the virtual environment. 


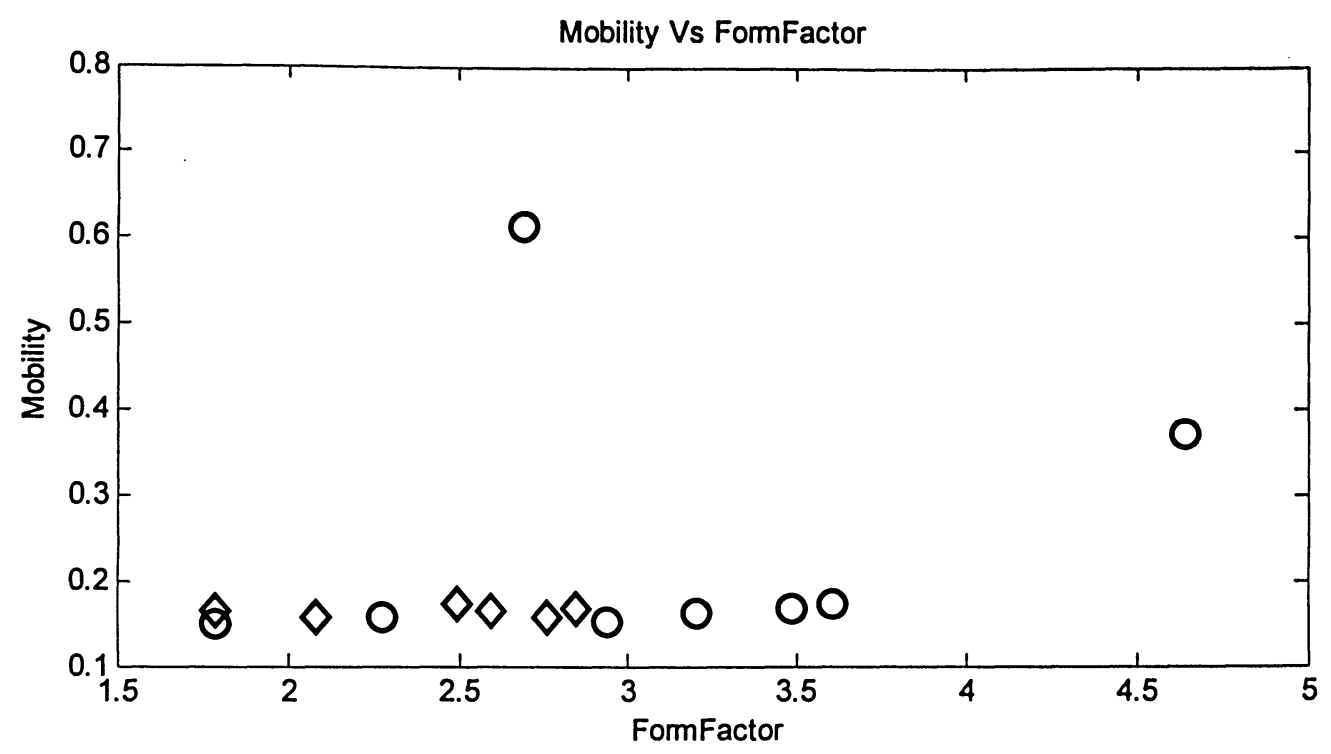

Figure F.6: The figure shows the mobility plotted against form factor for crouches and jumps. The diamonds are jumps and the circles are crouches. Both jumps and crouches are taken from random trials in the virtual environment. 


\section{Appendix G}

\section{Detailed graphs of cross-correlation of}

\section{visual signal to the buffer signals}

In this appendix graphs showing the cross correlation of visual signal (used for the model, chapter 5, Figure [30]) with the data stored in the memory (individual signals as explained in Figure [30]) are shown.

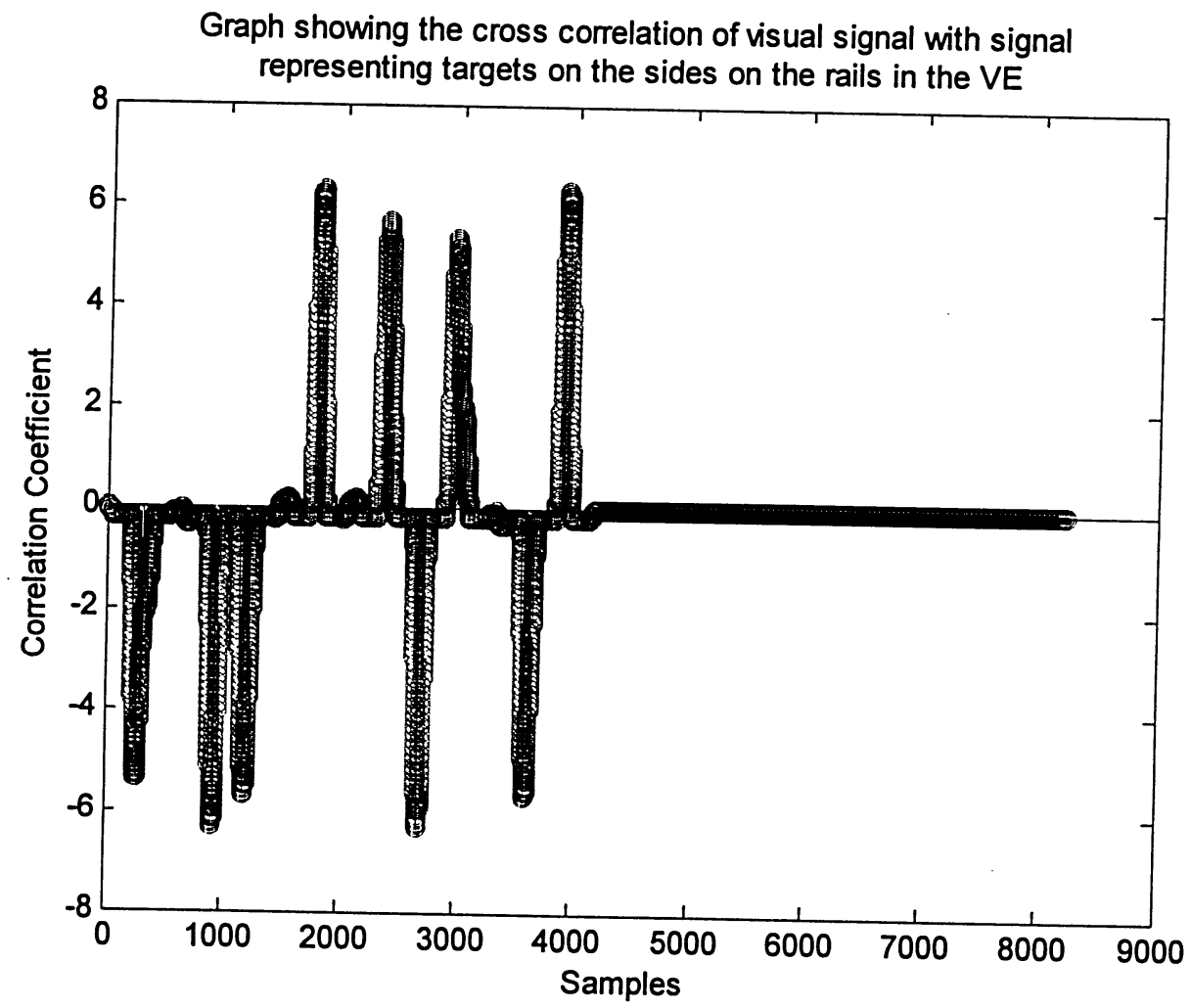

Figure G.1: Graph showing the cross correlation of visual signal with signal representing targets on the sides on the rails in the VE. 


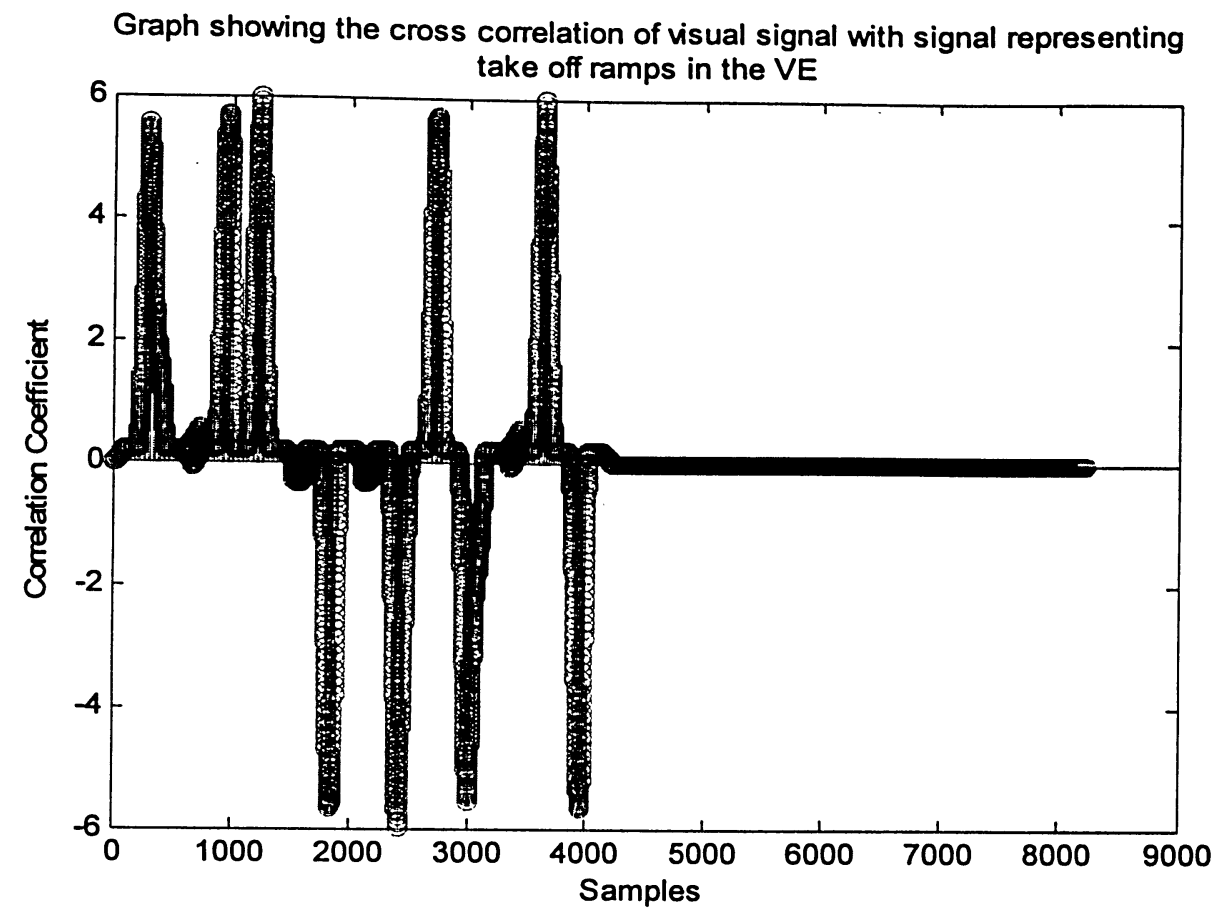

Figure G.2: Graph showing the cross correlation of visual signal with signal representing take off ramps in the VE.

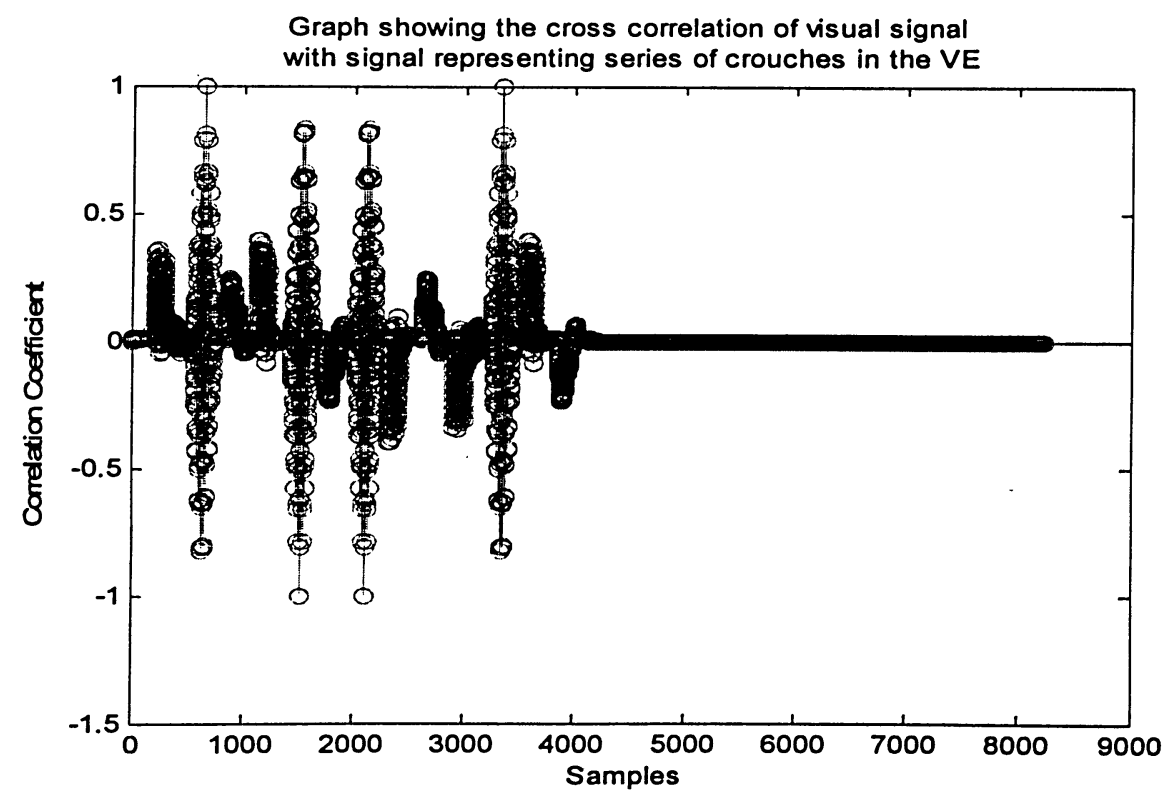

Figure G.3: Graph showing the cross correlation of visual signal with signal representing series of crouches in the VE. 


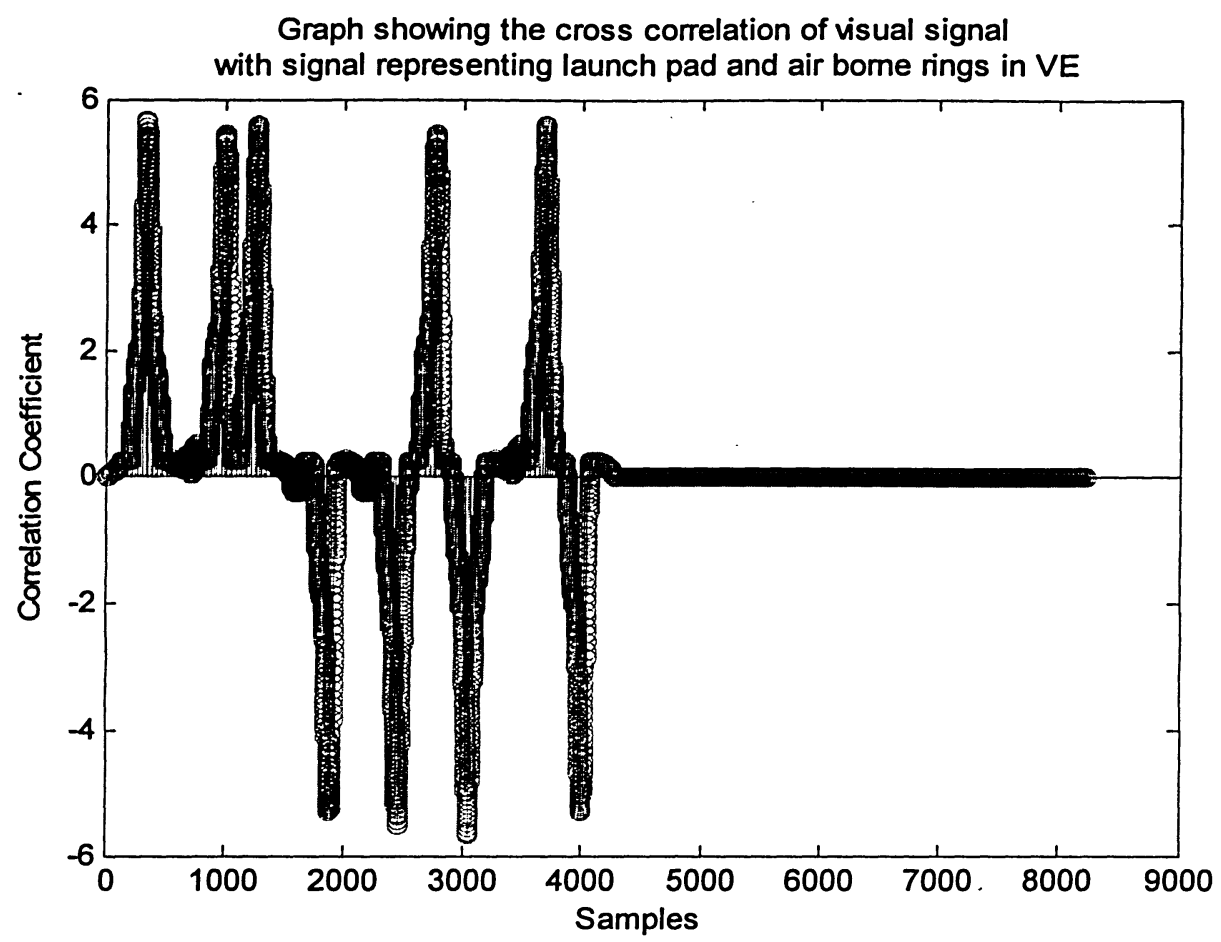

Figure G.4: Graph showing the cross correlation of visual signal with signal representing launch pad and air borne rings in VE. 


\section{Publications}

1) Sumandeep Virk and Kristiina McConville. Human sensory systems and Balance Stability. ICUE, International Conference for Upcoming Engineers, Waterloo, May 13-14, 2006.

2) Sumandeep Virk and Kristiina McConville. Applications of virtual reality in occupational falls rehabilitation. OOHNA, Ontario Occupational Health Nurses Association, Toronto, May 30-June 2, 2006.

3) Sumandeep Virk and Kristiina McConville. Virtual Reality Applications in Improving Postural Control and Minimizing Falls. EMBS, Engineering in Medicine and Biology Society, NY, Aug 30- Sept. 3, 2006.

4) Sumandeep Virk and Kristiina McConville. Virtual Reality: Applications in minimizing occupational falls. ACE, Association of Canadian Ergonomists, Banff, October 22- 25, 2006.

5) Sumandeep Virk and Kristiina McConville. Head movements and balance analysis in a virtual environment for reduced falls and a safer occupational environment. IAPA, Industrial Accident Prevention Association, Toronto, April 16-18, 2007.

6) Kristiina M. Valter McConville.and Sumandeep Virk. Visual motion, head and body movements evaluated in a virtual environment for vestibular rehabilitation. IEEE EMBS Conference on Neural Engineering. Hawaii, May 2-5, 2007. 
7) S. Virk, C.-C. (L.) Kant, M. Milosevic, D.M. Mravyan, B. Grabovac, M. Khodaei, C. Chao, R. Karkokli, R. Li, K. Valter McConville. Improvement in balance with a costeffective virtual reality environment.FICCDAT, Festival of international conferences on care giving, disability, aging and technology, Toronto, June 16-19, 2007. 


\section{Bibliography}

[1] Hayes ,W.C., Myers,E.R., Robinovitc, S.N. h, Kroonenberg, A. Van Den., Courtney,A.C., and Mcmahon, T.A. Etiology and prevention of age-related hip fractures. Bone. Vol. 18, 77S86S, 1996.

[2] Maki , B., Mcllroy W. Change in Support Balance Reactions in Older Persons: An Emerging Research Area of Clinical Importance. Neurol Clin 23 (2005) 751-783.

[3] Tinetti, M.E .,and Williams , C.S. Falls, injuries due to falls, and the risk of admission to a nursing home. New Engl. J. Med. Vol 337, 1279-1284, 1997.

[4] Yim-Chiplis K P, Talbot A. L. Defining and Measuring Balance in Adults. Biological Research for Nursing. Vol 1, No. 4, April 2004, 321-331.

[5] Bles W, Vianney de Jong JM, de Wit G (1983) Compensation for labyrinthine defects examined by use of a tilting room. Acta Otolaryngol 95:576-579.

[6] Peterka RJ (2002) Sensorimotor integration in human postural control. J Neurophysiol 88:1097-1118.

[7] Peterka RJ, Benolken MS (1995) Role of somatosensory and vestibular cues in attenuating visually induced human postural sway. Exp Brain Res 105:101-110. 
[8] Mergner T., Schweigart G., Maurer C., Blümle A. Human postural responses to motion of real and virtual visual environments under different support base conditions, Experimental Brain Research, Volume 167, Issue 4, Dec 2005, Pages 535 - 556.

[9] Bos E. J, Bles W, Hosman R, Groen L E. The cause of spatial disorientation. RTO HFM symposium on "Spatial Disorientation in Military Vehicles: Causes, Consequences and Cures". Spain 15-17 April 2002.

[10] Proudlock F A, Shekhar H, Gottlob I. Age related changes in head and eye coordination. Neurobiology of aging 25 (2004) 1377-1385.

[11] Paulus W.M, Straube A, Brandt Th. Visual stabilization of posture: Physiological stimulus characteristics and clinical aspects. Brain 1984, 107(4):1143-1163.

[12] Fetter M, Zee DS. Recovery from unilateral labryrinthectomy in rhesus monkey. Journal of Neurophysiology 1988, 59 (2): 370-393.

[13] Miles FA, Eighmy BB. Long term adaptive changes in primate vestibuloocular reflex. I. Behavioral observations. Journal of Neurophysiology 1980, 43 (5):1406-1425.

[14] Tinetti ME, Speechley M, Ginter SF. Risk factors for falls among elderly persons living in the community. N Engl J Med 1988; 319:1701-7. 
[15] Overstall PW, Exton-Smith AN, Imms FJ, Johnson AL. Falls in the elderly related to posture imbalance. Br Med J 1977; 1:261-4.

[16] Helen Cohen. Changes in sensory organization test scores with age. Age and Ageing. Jan 1996. FindArticles.com. 22 Jan. 2007.

[17] Paul F. Smith, Yiwen Zheng, Arata Horii , Cynthia L. Darlington .Does vestibular damage cause cognitive dysfunction in humans. Journal of Vestibular Research Issue: Volume 15, Number 1 / 2005 Pages: $1-9$.

[18] Quant S, Adkin AL, Staines WR, Maki BE, Mcllroy WE. The effect of a concurrent - cognitive task on cortical potentials evoked by unpredictable balance perturbations. BMC Neurosci. May 17;5:18, 2004.

[19] John L. Zettel, Andrea Holbeche, William E. McIlroy, Brian E. Maki, Redirection of gaze and switching of attention during rapid stepping reactions evoked by unpredictable postural perturbation, Experimental Brain Research, Volume 165, Issue 3, Sep 2005, Pages 392 - 401.

[20] Keshner, E. A., \& Kenyon, R. V. (2000). The influence of an immersive virtual environment on the segmental organization of postural stabilizing responses. Journal of VestibularResearch, 10, 201-219. 
[21] Mergner T., Schweigart G., Maurer C., Blümle A. Human postural responses to motion of real and virtual visual environments under different support base conditions, Experimental Brain Research, Volume 167, Issue 4, Dec 2005, Pages 535 - 556.

[22] Sparto P, Whitney S, Hodges L, Furman J, Redfern M. Simulator sickness when performing gaze shifts within a wide field of view optic flow environment: preliminary evidence for using virtual reality in vestibular rehabilitation. J NeuroEngg and Rehabilitation 2004; 1:14.

[23] Lestienne F, Soechting J, Berthoz A. Postural readjustments induced by linear motion of visual scenes. Exp Brain Res 28:363-384.

[24] Miles FA, Eighmy BB. Long term adaptive changes in primate vesibuloocular reflex. I. Behavioral observations. J Neurophysiol 1980, 43(5). 1406-1425.

[25] Fetter M, Zee DS. Recovery from unilateral labyrinthectomy in rheus monkey. J Neurophysiol 1988, 59 (2), 370-393.

[26] Shepard NT, Telian SA, Smith -Wheelock M. Habituation and balance retraining therapy. A retrospective review. Neural Clin 1980, 8(2). 459-475.

[27] Jacob RG, Woody SR, Clark DB, Lilienfeld SO. Discomfort with space and motion. A possible marker of vestibular dysfunction assessed by the situational characteristic questionnaire. Journal of Psychopathology \& Behavioral Assessment 1993, 15:299-324. 
[28] Bronstein AM: The visual vertigo syndrome. Acta Otaloryngol (Stockh) 1995:45-48.

[29] Lee DN, Lishman JR. Visual proprioceptive control of stance. J Hum Mov Stud 1:87-95. 1975.

[30] Hayashi R, Miyake A, Watanabe S. The functional role of sensory inputs from the foot: stabilizing human standing posture during voluntary and vibration-induced sway. Neurosci. Res 1998; 5:203-213.

[31] Kooij H.V, Jacobs R, Koopman B, Helm F, V. An adaptive model of sensory integration in a dynamic environment applied to human stance control. Biol. Cybern 84, 103-115 (2001).

[32] Bos J, E., and Bless W. Theoretical considerations on canal-otolith interaction and an observer model. Biol. Cybern 86, 191-207(2002).

[33] Bles W, Vianney JM, De Wit G. Compensation for labyrinthine defects examined by use of tilting room. Acta Otolaryngol. 95:576-579. 1983.

[34] Kuo D. A. An optimal state estimation model of sensory integration in human postural balance. J neural Engg 2 (2005) S235-S249.

[35] Kooij V H., Jacobs R, Koopman B, Grttoenboer H. A multisensory integration model of human stance control. Biol. Cybern. 80, 299-308(1999). 
[36] Oie K, Kiemel T, Jeka J J. Multisensory fusion: Simultaneous re-weighting of vision and touch for the control of human posture. Cognitive Brain Research 14 (2002) 164-176.

[37] Carver S, Kiemel T, Jeka J. Modeling the dynamics of sensory reweighting. Biol. Cybern. 95, 123-134(2006).

[38] Musolino M. Postural Sway and sway-adaptation during exposure to optic flow: The effect of stimulus periodicity and concurrent cognitive tasks. Thesis (Ph.D). School of Engineering, University of Pittsburg. 2006.

[39] Davidson PR, Jones RD, Andrea JH, and Sirisena HR. Simulating closed and open loop voluntary movement: a non Inear control systems approach. IEEE Transactions on Biomedical Engineering 49(11), 1242-52.2002.

[40] Fitzpatrick R, Burke D and Gandevia SC. Loop gain of reflexes controlling human standing measured with the use of postural and vestibular disturbances. J Neurophysiol 76, 39994-40008.

[41] Travis R C. An experimental analysis of dynamic and static equilibrium. Journal of Experimental Psychology (1945), 35, 216-234.

[42] Edwards A S. Body sway and vision. Journal of Experimental Psychology (1946), 36, 526535. 
[43] Paulus W.M, Straube A, Brandt Th. Visual stabilization of posture. Physiological stimulus characteristics and clinical aspects. Brain 107.1143-1163.

[44] Weiss P, Rand D, Katz N, and Kizony R. Video Capture Virtual reality as a flexible and effective rehabilitation tool. Journal of NeuroEngineering and Rehabilitation. 2004. I:12. doi:10.1186/1743-0003-1-12.

[45] Vouriot A, Gauchard G, Chau N, Benamghar L, Lepori ML, Mur JM, Perrin P. Sensorial organization favoring higher visual contribution is a risk factor of falls in an occupational setting. NeuroSci Res 2004; 48:239-247.

[46] Paillard Th, Noe F. Effect of expertise and visual contribution on postural control in soccer. Scadinavian J of Med \& Sci in sports. 2005.

[47] Kim N, Yoo C, and Im J. A new rehabilitation system for postural balance control using virtual reality technology. IEEE transactions on rehabilitation engineering, Vol 7, No. 4, Dec. 1999.

[48] Schultheis MT, Rizzo AA: The application of virtual reality technology in balance rehabilitation. Rehabilitation Psychology 2001, 46:296-311. 
[49] Alpini D, Pugnetti L, Mendozzi L, Barbieri E, Monti B, and Cesarani A. Virtual reality in vestibular diagnosis and rehabilitation. Proc. 2nd Euro. Conf. disability, virtual reality \& Assoc. Tech.,Skovde, Sweden, 1998.

[50] Gray, Henry. Pick, T. Pickering, \& Howden, Robert (Eds.) (1995). Gray's Anatomy (15th ed.). New York: Barnes \& Noble Books.

[51] Luchies C.W., Alexander N.B., Schultz A.B., and J. Ashton-Miller. Stepping responses of young and old adults to postural disturbances: kinematics. J. Amer. Geriatr. Soc., vol. 42, pp. 506-512, 1994.

[52] McIlroy W.E., and Maki B.E.. Age-related changes in compensatory stepping in response to unpredictable perturbations. J. Gerontol., vol. 51A, pp. M289-M296,1996.

[53] Maki B.E., Edmondstone M.A., and Mcllroy W.E.. Age-related differences in laterally directed compensatory stepping behavior. J. Gerontol., vol. 55A, pp. M270-M277, 2000.

[54] Maki BE, Mcllroy WE, Fernie GR. Change-in-support reactions for balance recovery. IEEE Eng Med Biol Mag. 2003 Mar-Apr;22(2):20-6. 
[55] Pyykko I, Aalto H, Hytonen M, Starck J, Jantti P, Ramsay H.1988. Effect of age on postural control. In: Amblard B, Berthoz A, Clarac F.(Eds), Proceedings of the ninth International Symposium on postural and gait research, International Congress series 812, Posture and Gait: development, Adaptation and Modulation. ExcerptaMedica, Marseille, France, Amsterdam, New York, Oxford, pp 95-104.

[56] Kennedy RS, Lane NE, Berbaum KS, Lilienthal ML. Simulator sickness questionnaire: An enhanced method for quantifying simulator sickness. International Journal of Aviation Psychology 1993, 3:203-220.

[57] Cobb SVG, Nichols S, Ramsey A, Wilson JR. Virtual reality-induced symptoms and effects (VRISE). Presence: Teleoperators and virtual environments 1999, 8(2):169-186.

[58] Kennedy RS, Stanney KM, Dunlap WP. Duration and exposure to virtual environments: simulator sickness curves during and across sessions. Presence: Teleoperators and virtual environments 2000, 9(5):463-472.

[59] Newell KM, Liu, Y.-T., Mayer-Kress, G. Time Scales in Motor Learning and Development. Psychological Review. 2001. 108(1): 57-82.

[60] Bugnariu N, Fung J. Aging and selective sensorimotor strategies in the regulation of upright balance. $5^{\text {th }}$ International workshop on virtual rehabilitation. NY, Aug 29-30, 2006. 
[61] Rangayyan M. R. Biomedical Signal Analysis. John Wiley \& Sons, Inc., 2002.

[62] Osu R, Franklin W. D., Kato H, Gomi H, Domen K, Yoshioka T, and Kawato M. Short- and Long-Term changes in joint co-contraction associated with motor learning as revealed from surface EMG. J Neurophysiol. 88:991-1004,2002.

[63] Woollacott M, and Shumway-Cook A. Attention and control of posyure and gait: a review of an emerging area of research. Gait and Posture 16:1-14. 2002.

[64] Lord S.R, Sherrington C and Menz H.B. Falls in older people: risk factors and strategies for prevention. NY: Cambridge University Press. 2001.

[65] Gallagher S.P and Lajoie Y. Predicting falls within elderly community: comparison of postural sway, reaction time, the berg balance scale and the Activities-specific balance confidence (ABC) scale for comparing fallers and non-fallers. Arch Genontol Geriat 38:11-26. 2004.

[66] Evans WJ. Exercise training guidelines for the elderly. Med Sci Sport Exer 32:12-17. 1999.

[67] Bisson E, Contant B, Sveistrup H, and Lajoie Y. Functional balance and dual-task reaction times in older adults are improved by virtual reality and biofeedback training. CyberPsychology \& Behavior. Vol 10 (1), 2007. 
[68] Keshner E A, Streepey J, Dhaher Y, and Hain C. T. Pairing virtual reality with dynamic posturography serves to differentiate patients with visual vertigo. $5^{\text {th }}$ International Workshop on virtual rehabilitation. NY, August 29-30, 2006.

[69] Grabovac B, Mravyan D. Portable ECG and EMG Monitor. ICUE, International Conference for Upcoming Engineers, Waterloo, May 13-14, 2006.

[70] Milosevic, M., and McConville, K. Falls Prevention among Older Adults through Rectification of Hand Movements Based on a Novel Method for Quantitative Balance Assessment. FICCDAT - Festival of International Conferences on Caregiving, Disability, Aging and Technology, Toronto, 2007.

[71] Carver S, Kiemel T, Kooij H. V, Jeka. J.J. Comparing internal models of the dynamics of the visual environment. Biol. Cybern. 92, 147-163 (2005). 